\title{
Strategy-proofness and single-dipped preferences
}

Citation for published version (APA):

Öztürk, M. (2013). Strategy-proofness and single-dipped preferences. [Doctoral Thesis, Maastricht University]. Datawyse / Universitaire Pers Maastricht. https://doi.org/10.26481/dis.20131108mo

Document status and date:

Published: 01/01/2013

DOI:

10.26481/dis.20131108mo

Document Version:

Publisher's PDF, also known as Version of record

\section{Please check the document version of this publication:}

- A submitted manuscript is the version of the article upon submission and before peer-review. There can be important differences between the submitted version and the official published version of record.

People interested in the research are advised to contact the author for the final version of the publication, or visit the DOI to the publisher's website.

- The final author version and the galley proof are versions of the publication after peer review.

- The final published version features the final layout of the paper including the volume, issue and page numbers.

Link to publication

\footnotetext{
General rights rights.

- You may freely distribute the URL identifying the publication in the public portal. please follow below link for the End User Agreement:

www.umlib.nl/taverne-license

Take down policy

If you believe that this document breaches copyright please contact us at:

repository@maastrichtuniversity.nl

providing details and we will investigate your claim.
}

Copyright and moral rights for the publications made accessible in the public portal are retained by the authors and/or other copyright owners and it is a condition of accessing publications that users recognise and abide by the legal requirements associated with these

- Users may download and print one copy of any publication from the public portal for the purpose of private study or research.

- You may not further distribute the material or use it for any profit-making activity or commercial gain

If the publication is distributed under the terms of Article $25 \mathrm{fa}$ of the Dutch Copyright Act, indicated by the "Taverne" license above, 
Strategy-proofness and Single-dipped Preferences 
Strategy-proofness and Single-dipped Preferences

(C) Copyright M Ozturk, Maastricht 2013

All rights reserved. No part of this publication may be reproduced, stored in a retrieval system, or transmitted, in any form, or by any means, electronic, mechanical, photocopying, recording or otherwise, without the prior permission in writing from the author.

This book was typeset by the author using $\mathrm{LT}_{\mathrm{E} X}$.

Published by Universitaire Pers Maastricht

ISBN 9789461592699

Printed in the Netherlands by Datawyse 


\title{
Strategy-proofness and Single-dipped Preferences
}

\author{
Proefschrift
}

ter verkrijging van de graad van doctor aan de Universiteit Maastricht, op gezag van Rector Magnificus,

Prof. dr. L.L.G. Soete,

volgens het besluit van het College van Decanen, in het openbaar te verdedigen op vrijdag 8 november 2013, om 12:00 uur

door

Murat Öztürk

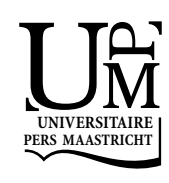




\section{Promotor:}

Prof. dr. Hans (J.M.) Peters

\section{Copromotor:}

Dr. Ton (A.J.A.) Storcken

Beoordelingscommissie:

Prof. dr. Rudolf (J.) Müller (voorzitter)

Dr. Burak Can

Prof. dr. Bettina Klaus (University of Lausanne)

Prof. dr. Harrie (C.M.) de Swart (Erasmus University Rotterdam) 


\section{Acknowledgements}

The dissertation you are holding is the result of an academic and personal journey, which would not have been possible without support. Some of the people around me guided me through the journey, some walked next to me, and, while some pushed me forward. It is an opportunity for me to express my sincere gratitude to all of them.

My journey started when I was a mathematics senior and enthusiastically attended the Game Theory lectures taught by Farhad Husseinov. He helped me to realize the existence of mathematical studies that I can enjoy. Thanks to his guidance, I decided to continue my journey at İstanbul Bilgi University, where I got chance to work on Social Choice Theory with Remzi Sanver, whom I always appreciate his valuable contribution to my academic knowledge. I wish to emphasize thankfulness to him for giving me the chance to be one of his students and inspiring me about academia and life. Along with him, I would like to say thank you to the following nice people I met at İstanbul Bilgi University for their contributions; Göksel Aşan, Jean Laine, Ayça Ebru Giritligil, Fatma Aslan, Hande Oruç and Ali İhsan Özkes.

After receiving masters degree, I was able to join Maastricht University as a Ph.D. candidate. The three wonderful years I spent in the department of Quantitative Economics was a unique experience. I would like to express my deepest appreciation to Hans Peters and Ton Storcken, my research supervisors, for their patient guidance, encouragement and useful critiques. Writing this thesis would not have been possible without their help and support. Moreover, they have taught me how to conduct a good academic 
research.

I am very grateful to evaluation committee, Rudolf Muller, Harrie de Swart, Bettina Klaus and Burak Can for their valuable critiques and comments. I also want to thank Graduate School of Business and Economics (METEOR) for funding my Ph.D. at Maastricht University.

Even though Maastricht is located thousands of kilometers away from my hometown, thanks to the Turkish community that never let me feel homesick. I want to thank them, namely Hande Karabıyı,, Seher Fazlıŏlu, Birol Yüceoğlu, Çiğdem Akbulut, Özge Gökdemir Dumludă̆, Devrim Dumludağ, Mehmet İsmail, and Emel Öztürk for the cheerful lunch times and long coffee breaks.

I wish to thank my parents and parents-in-law, for their support and encouragement throughout my study. Last but not the least; my beloved wife Sena Demet was always with me along the way. Hereby, I would like to emphasize that the love we share is the biggest motivation I had in these years.

Murat Öztürk

Maastricht, September 2013 


\section{Contents}

1 Introduction 1

2 Polytopes $\mathbf{5}$

2.1 Introduction . . . . . . . . . . . . . . . 5

2.2 Notations, basic model, and preliminaries . . . . . . . . . 9

2.2.1 Notations . . . . . . . . . . . . . . . 9

2.2.2 The basic model ................ . 9

2.2.3 Preliminary results .............. 10

2.3 Dictatorship .................... . . 12

2.4 Nondictatorship . . . . . . . . . . . . . . . . 27

$2.4 .1|\mathcal{B}|=2$. . . . . . . . . . . . . . . . . . . . 28

2.4.2 $\mathcal{B}$ is rectangular . . . . . . . . . . . . . . . 32

2.5 Concluding remarks ................. . 32

3 Disc $\quad 35$

3.1 Introduction and model . . . . . . . . . . . . . . 35

3.2 Dictatorship ................... . 36

4 Trees $\quad 43$

4.1 Introduction . . . . . . . . . . . . . . . . . . . 43

4.2 Basic Notations, The Model and Preliminaries . . . . . . . 44

4.2.1 Notations ................... 45

4.2.2 The model .................. 46

4.2.3 Preliminaries ................ . 47

4.3 Pairwise Monotonicity ............... . 53 
5 Two Neighboring Countries $\quad 57$

5.1 Introduction . . . . . . . . . . . . . . 57

5.2 Basic Notations and the Model . . . . . . . . . . . 58

5.3 The case $\beta=0 \ldots \ldots \ldots \ldots \ldots$. . . . . . . . . 61

5.4 The case $\beta \in] 0,1[,|N|=2 \ldots \ldots \ldots \ldots 62$

5.5 The case $\beta \in] 0,1[,|N|>2 \ldots \ldots \ldots \ldots 6$

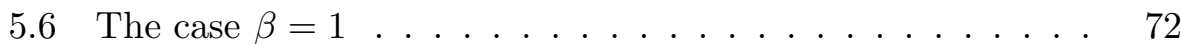

5.6 .1 Concluding Remarks . . . . . . . . . . . . . 74

$\begin{array}{ll}\text { Bibliography } & 79\end{array}$

Nederlandse Samenvatting (Dutch summary) 80

$\begin{array}{ll}\text { Curriculum Vitae } & 84\end{array}$ 


\section{Chapter 1}

\section{Introduction}

Modern social choice theory is acknowledged to start with Arrow's impossibility theorem, which says there is no non-dictatorial social choice rule (solution) that satisfies certain desirable conditions. This discouraging impossibility result encouraged researchers to investigate how strong this result is and if there are ways to avoid this result. Another famous impossibility result is that of Gibbard (1973) and Satterthwaite (1975). The Gibbard-Satterthwaite theorem says that there is no non-dictatorial solution that picks an outcome for any preference profile and satisfies Pareto optimality and strategy-proofness. Pareto optimality says that there is no other alternative which is as good as the outcome for everyone and strictly better for some. Strategy-proofness ensures that no agent can obtain a better outcome for himself by misrepresenting his preference, i.e. no agent has an incentive to manipulate. This dissertation aims to investigate the outcomes of weakening one of the assumptions: the unrestricted domain. In particular, we will consider so-called single-dipped preferences.

In 1995, citizens of Orange County met to discuss the new landfill that was planned to be built. Wistar (1995), while writing an article about opinions of these citizens, starts his article with this sentence: 
"Not in my backyard" was the message shared by the crowd that gathered in Chapel Hill to discuss the new Orange County Landfill.

The idea of "Not in my backyard" (NIMBY) comes from the concept of public bad. A public bad is a facility that is needed in society, but no one wants to have it close by. With respect to public bads, one may ask the following: Let there be three possible locations for the landfill: (1) the street that the agent lives in, (2) the neighboring street and (3) a street that is miles away. Clearly, most agents will prefer the third option. The nature of a public bad leads to the idea of single-dipped preferences. An agent has a location in mind that is the worst possible location, the dip of the agent, to locate this public bad and the preference of the agent increases as the public bad moves further away from this dip. This dissertation aims to investigate, characterize if possible, solutions that satisfy Pareto optimality and strategy-proofness when a finite number of agents have single-dipped preferences over different sets of alternatives. It consists of self-contained chapters that are organized according to different sets of alternatives: a polytope in the Euclidean plane, a disc, a tree shaped network and a closed interval on the real line. Moreover, this dissertation considers situations with one public bad, except in the last chapter. In the last chapter, two neighboring countries each locate a public bad on their territory.

\section{Outlines of Chapters 2-5}

In Chapter 2, a model with finitely many agents, who have single-dipped Euclidean preferences on a polytope in the Euclidean plane, is considered. A solution assigns to each profile of reported dips a point of the polytope. A point $x$ of the polytope is called single-best if there is a point $y$ of the polytope such that $x$ is the unique point of the polytope at maximal distance from $y$. It is proved that if the polytope has exactly two single-best points or it has exactly four single-best points which form the vertices of a rectangle, then there are nondictatorial strategy-proof and Pareto optimal rules, which can be described by committee voting (simple games) between the single-best alternatives. In all other cases, Pareto optimality and strategy-proofness imply dictatorship. This framework models situa- 
tions where public bads such as garbage dumping grounds or nuclear plants have to be located within a confined region.

In Chapter 3, a model with finitely many agents, who have single-dipped Euclidean preferences on a disc in the Euclidean plane, is considered. A solution assigns to each profile of reported dips a point of the disc. It is proved that any strategy-proof and Pareto optimal rule is dictatorial. This framework models situations where public bads such as garbage dumping grounds or nuclear plants have to be located within a confined region that has the shape of a disc.

In Chapter 4, a model with finitely many agents, who have single-dipped preferences on a tree network, is considered. A solution assigns to each profile of reported dips a point on a tree network. An extreme leaf is an end point of a path with maximal length. We show that the outcome of any non-corrupt, strategy-proof, Pareto optimal and anonymous solution for any profile is an extreme leaf. Also we show the equivalence of these four conditions and pairwise monotonicity.

In Chapter 5, a model with finitely many agents, who have single-dipped preferences on a one-dimensional region formalized by a closed interval, is considered. Moreover, in a neighboring one-dimensional region, in fact an adjacent closed interval, a public bad is located. A solution assigns to each profile of reported dips a point in the region. We characterize solutions that satisfy desirable conditions for each possible location of the public bad of the neighboring region. 



\section{Chapter 2}

\section{Polytopes}

\section{$2.1 \quad$ Introduction}

Consider the problem of locating a windmill park, garbage dumping ground, heavy industry, or nuclear plant within a confined area, such as a city, province, or country. ${ }^{1}$ These are examples of public bads: people agree on their usefulness or even necessity but typically do not want them in their backyards. In this chapter we assume that the public bad is to be located within a given region - a subset of the plane - and that the location is determined by voting among a set of agents (for instance, inhabitants of the region, or political representatives). Each agent is characterized by a Euclidean single-dipped preference: there is a worst point, the dip, which is a specific point of the region, and preference increases with the Euclidean distance from this dip. A vote then consists of reporting one's dip. Typically, what an agent regards to be his dip is private knowledge: it could coincide with his residence, the school of his children, an important natural resource, etc. In order for the location of the public bad to be based on the right information about the agents' preferences the (voting) rule should be strategy-proof: no agent should be able to achieve a location farther away from his true dip by not reporting truthfully. Also, we will

\footnotetext{
${ }^{1}$ This chapter is based on Öztürk, Peters, and Storcken (2012).
} 
require the rule to be Pareto optimal: it should not assign a location for which there is another location at least as distant for all and strictly farther away for some agents, given the reported dips.

In this chapter we assume that the region is a two-dimensional polytope $A$, i.e., the convex hull of at least three but finitely many points, which are the extreme points of the polytope. An important concept is that of a single-best point, i.e., a point of $A$ that is the unique point at maximal distance from some other point of $A$. Single-best points are always extreme points, although not every extreme point is necessarily a single-best point. For instance, if the boundary of $A$ is a triangle with vertices $a, b$, and $c$, then these three points are the single-best points if the triangle is acute. However, if the angle at $a$ is right then $a$ can be a best point but never a single-best point, and if this angle is obtuse then $a$ is not even a best point.

We assume that there are $n \geq 2$ agents; a profile is a vector of $n$ points of $A$ - interpreted as a vector of reported dips - and a rule assigns a point of $A$ to each profile. Our main results are as follows. If $A$ does not have exactly two single-best points or if $A$ does not have exactly four single-best points which form the vertices of a rectangle, then a strategy-proof and Pareto optimal rule must be dictatorial: there is a fixed agent $d$ such that the rule always assigns a point at maximal distance from d's dip. This result is obtained by proving that the set of decisive coalitions is an ultrafilter and, in particular, closed under taking intersections. The last property does not hold if $A$ has exactly two single-best points or exactly four single-best points which are the vertices of a rectangle. For the case that $A$ has exactly two single-best points $a$ and $b$-which means, roughly, that $A$ is sufficiently flat with $a$ and $b$ as 'end points' - we characterize all strategy-proof and Pareto optimal rules under a few mild additional (tie-breaking) assumptions: such rules are described by committee (simple game) voting between $a$ and $b$. Similarly, for the case where $A$ has exactly four single-best points which are the vertices of a rectangle, we can obtain strategy-proof and Pareto optimal rules by committee voting between the upper and lower and between the left and right vertices of the rectangle; for this case we content ourselves by giving an example. 
The message of the chapter is therefore that dictatorship can be avoided if the region is sufficiently flat (in terms of width) or if we can identify four locations which are the vertices of a rectangle and which are the only single-best points. Although there is certainly some hindsight intuition for these results - like a small induced preference domain in the flat case, and separability of preferences along axes in the rectangular case - the proofs are far from trivial.

Since some parts of the proof rely on the finiteness of the set of extreme points of a region $A$, extension of our results to general compact convex set does not seem straightforward. In Chapter 3 we show that dictatorship continues to hold when $A$ is a disc (a circle and its inside) but this needs a separate proof.

Related literature To the dictatorship part of our results the classical work of Gibbard (1973) and Satterthwaite (1975) does not directly apply since we do not have full preference domains. An exception is the acute triangle: if we know that the rule assigns a vertex then in this case dictatorship follows from Gibbard-Satterthwaite, since single-dipped preferences generate the full domain of six (strict) preferences over the vertices (see the end of Section 2.3).

In one dimension, single-dipped and single-peaked preferences are both special cases of value restriction (Inada, 1964), but it is well known that the consequences for Pareto optimal and strategy-proof rules are quite different. Also in spatial models like ours this is the case. In particular, under single-dipped preferences, strategy-proofness and Pareto optimality force the outcome of the rule to be on the boundary of the region under consideration - which is also why the restriction to compact sets is natural in the present framework. As a result, under single-dipped preferences much is going to depend on the shape of the boundary of the region. Typically, also, continuity of a rule is not a condition that can be imposed since even a dictatorial rule is not continuous: a small change in the preference (dip) of the dictator may cause the outcome to swap to the opposite side of the region. Generally speaking, single-peakedness of preferences seems to allow for more possibilities and to lead less frequently to dictatorship (Black, 
1948; Moulin, 1980; Border and Jordan, 1983).

In spite of its apparent relevance, existing work on collective decision making in the presence of single-dipped preferences is relatively limited. As far as we know ours is the first paper to study strategy-proofness under single-dipped preferences in two-dimensional space. The one-dimensional case has been studied before. Peremans and Storcken (1999) characterize all strategy-proof rules for the case that $A$ is an arbitrary subset of the real line and preferences are single-dipped (and not necessarily symmetric). An implication of their work is that individual and coalitional strategy-proofness are equivalent in this model. Also Barberà, Berga, and Moreno (2012) reach this conclusion and sharpen the bounds on the range of strategy-proof rules, depending on different subclasses of single-dipped preferences. Manjunath (2009) specifically studies unanimous and strategyproof rules defined on a real interval. Thus, all these works concern the location of a public bad where the region $A$ is one-dimensional.

The private good case with single-dipped preferences is considered in Klaus, Peters, and Storcken (1997), which deals with the division of a perfectly divisible commodity. Klaus (2001b) considers the case where this commodity is an indivisible object and has to be allocated to one of the agents. Ehlers (2002) considers the probabilistic allocation of an indivisible object.

The proof of the dictatorship result based on decisive coalitions and ultrafilters has been used before, see Kirman and Sondermann (1972) and Hansson (1976).

Finally, there is a - more extensive - literature on mechanism design for the location of public bads (obnoxious facilities) when monetary transfers are allowed, see for instance Kunreuther and Kleindorfer (1986) or, more recently, Lescop (2007) or Besfamille and Lozachmeur (2010).

Organization of the chapter Section 2.2 starts with notations, the basic model, and some preliminary results. In Section 2.3 we derive the dictatorship result, and in Section 2.4 we consider the non-dictatorship cases. Section 2.5 concludes. 


\subsection{Notations, basic model, and preliminar- ies}

We start by fixing some notations to be used throughout the chapter.

\subsubsection{Notations}

For a subset $X$ of $\mathbb{R}^{2}$ (endowed with the usual topology) we denote by $\partial X$ its boundary, by $X^{\circ}$ its interior, and by $\bar{X}$ its closure.

Let $a, b, c \in \mathbb{R}^{2}$ be three different points. Then $[a, b]$ denotes the closed line segment between points $a$ and $b,] a, b[$ the (relatively) open line segment between $a$ and $b$, and $[a, b[$ and $] a, b]$ the two half open line segments. The midpoint of $[a, b]$ is denoted by $m_{a, b}$ and its perpendicular bisector by $\ell_{a, b}$. For $x \in \mathbb{R}^{2}$ we denote by $\sigma_{a, b}(x)$ the reflection of $x$ in the line $\ell_{a, b}$, i.e., $\ell_{a, b}$ is the perpendicular bisector of $\left[x, \sigma_{a, b}(x)\right]$.

If $a, b$, and $c$ are non-collinear we denote $m_{a, b, c}=\ell_{a, b} \cap \ell_{b, c} \cap \ell_{a, c}$ (and regard $m_{a, b, c}$ as a point rather than a set). Further, $\ell(a, b)$ denotes the straight line through the points $a$ and $b$.

The Euclidean distance between $a$ and $b$ is denoted by $\|a-b\|$. A straight line $\ell$ divides the plane $\mathbb{R}^{2}$ into two half-planes. For $a \notin \ell, H(\ell, a)$ indicates the closed half-plane containing $a$; we write $H^{\circ}(\ell, a)$ for the open halfplane, thus $H^{\circ}(\ell, a)=H(\ell, a)^{\circ}$. The circle with center $a$ and radius $r \geq 0$ is denoted by $\odot(a, r)$, hence $\odot(a, r)=\left\{x \in \mathbb{R}^{2}:\|a-x\|=r\right\}$. For $x, y \in \mathbb{R}^{2}$ on the same circle with center $a \in \mathbb{R}^{2}$, we write $[a ; x \frown y]$ for the arc between $x$ and $y$, that is, $[a ; x \frown y]=\odot(a,\|a-x\|) \backslash H^{\circ}(\ell(x, y), a)$ if $a$ is not on $\ell(x, y)$, otherwise $[a ; x \frown y]=\odot(a,\|a-x\|)$.

For an arbitrary set $D$ we denote by $|D|$ its cardinality.

\subsubsection{The basic model}

We next formulate the basic model. There is a finite set $N=\{1, \ldots, n\}$ $(n \geq 2)$ of agents and a compact subset $A \subseteq \mathbb{R}^{2}$ of alternatives. Each 
agent is endowed with a (Euclidean single-dipped) preference $R_{x}, x \in A$, defined as follows: for all $y, z \in A,(y, z) \in R_{x}$ if $\|x-y\| \geq\|x-z\|$. The alternative $x$ is the dip of preference $R_{x}$. Clearly, preference $R_{x}$ is completely determined by its dip; therefore, if convenient, we will denote a preference by its dip. An element $p=(p(1), \ldots, p(n)) \in A^{N}$, where $p(i)$ is the dip of agent $i$, is called a profile (of single-dipped preferences). A social choice function or simply rule is a map $\varphi: A^{N} \rightarrow A$. We are interested in rules that satisfy the following two properties. Rule $\varphi$ is called

[PO] Pareto optimal if for every profile $p \in A^{N}$, every agent $i \in N$, and every alternative $a \in A$ with $\|p(i)-a\|>\|p(i)-\varphi(p)\|$ there is an agent $j \in N$ such that $\|p(j)-a\|<\|p(j)-\varphi(p)\| ;$

[SP] strategy-proof if for every agent $i$ in $N$ and all profiles $p, q \in A^{N}$, with $q(j)=p(j)$ for all $j \in N \backslash\{i\}$, we have $\|p(i)-\varphi(p)\| \geq\|p(i)-\varphi(q)\|$.

The interpretation of Pareto optimality is as usual, and strategy-proofness (also as usual) implies that no agent can improve the outcome by misreporting his preference.

A subset of $N$ is called a coalition. As the reader may verify, strategyproofness is equivalent to the following condition. Rule $\varphi$ is called

[ISP] intermediate strategy-proof if for every coalition $S \subseteq N$ and all profiles $p, q \in A^{N}$ such that $p(j)=q(j)$ for all $j \in N \backslash S$ and there is an alternative $a$ with $p(i)=a$ for all $i \in S$, we have $\|a-\varphi(p)\| \geq\|a-\varphi(q)\|$.

Often ISP will be used instead of SP without explicit mentioning.

\subsubsection{Preliminary results}

Let $S \subseteq N$ be a coalition and let $a, b \in A$. By $\left(a^{S}, b^{N \backslash S}\right) \in A^{N}$ we denote the profile $p$ with $p(i)=a$ for all $i \in S$ and $p(i)=b$ for all $i \in N \backslash S$. (If $S=N$ we usually write $a^{N}$ instead of $\left(a^{N}, b^{\emptyset}\right)$.) To such a two-dip profile a Pareto optimal rule always assigns a boundary point, as the following lemma shows. 
Lemma 2.2.1. Let $a, b \in A, S \subseteq N$, and $p=\left(a^{S}, b^{N \backslash S}\right)$. Let $\varphi: A^{N} \rightarrow A$ be a Pareto optimal rule. Then $\varphi(p) \in \partial A$.

Proof. Let $\ell=\ell(a, b)$ if $a \neq b$ and let $\ell$ be an arbitrary straight line through $a$ if $a=b$. Let $x$ be the intersection of $\ell$ and the line $\ell^{\prime}$ through $\varphi(p)$ perpendicular to $\ell$. Now for points $c$ on $\ell^{\prime}$ such that $\left.\left.\varphi(p) \in\right] c, x\right]$, it follows that $\|a-c\|>\|a-\varphi(p)\|$ and $\|b-c\|>\|b-\varphi(p)\|$. Hence, by Pareto optimality we must have $c \notin A$, so that $\varphi(p)$ cannot be an interior point of $A$. Hence $\varphi(p) \in \partial A$.

Observe that the proof of this lemma does not use the compactness of the set $A$. Thus, the lemma justifies our restriction to compact sets: if $A$ is not bounded then a Pareto optimal rule does not exist, and if $A$ is not closed it may also fail to exist.

For a preference $R_{a}$, the set of best alternatives is denoted by $\mathfrak{b}\left(R_{a}\right)$ or by $\mathfrak{b}(a)$, i.e., $\mathfrak{b}\left(R_{a}\right)=\mathfrak{b}(a)=\{y \in A:\|a-y\| \geq\|a-z\|$ for all $z \in A\}$. A coalition $S \subseteq N$ is decisive (given a rule $\varphi$ ) if $\varphi(p) \in \mathfrak{b}\left(R_{a}\right)$ for all $a \in A$ and all $p \in A^{N}$ with $p(i)=a$ for all $i \in S$. Clearly, if $S$ is decisive and $S \subseteq T \subseteq N$, then $T$ is decisive. A rule $\varphi$ is dictatorial if there is a dictator, i.e., an agent $d \in N$ such that $\{d\}$ is decisive.

A collection $\mathcal{W}$ of subsets of $N$ is called an ultrafilter on $N$ if for all subsets $S, T \subseteq N$ we have (i) $\emptyset \notin \mathcal{W}$, (ii) if $S, T \in \mathcal{W}$, then $S \cap T \in \mathcal{W}$, and (iii) $S \in \mathcal{W}$ or $N \backslash S \in \mathcal{W}$. We have the following familiar property.

Lemma 2.2.2. Let $\mathcal{W}$ be an ultrafilter on $N$. Then there exists a unique $d \in N$ such that $\{d\} \in \mathcal{W}$.

Proof. By properties (i) and (ii) of an ultrafilter there can be at most one such $d$. If $N \backslash\{i\} \in \mathcal{W}$ for all $i \in N$ then $\cap_{i \in N} N \backslash\{i\} \in \mathcal{W}$ by (ii), but this violates (i) since $\cap_{i \in N} N \backslash\{i\}=\emptyset$. Hence, there must be a $d \in N$ with $N \backslash\{d\} \notin \mathcal{W}$, so by (iii) we have $\{d\} \in \mathcal{W}$. 


\subsection{Dictatorship}

Throughout this section, the set $A$ is a polytope with non-empty interior, i.e., the convex hull of a finite set $C=\left\{c_{1}, c_{2}, \ldots, c_{k}\right\} \subseteq \mathbb{R}^{2}$ with $k \geq 3$ where all alternatives in $C$ are extreme points of $A$. Further, $\varphi: A^{N} \rightarrow A$ is a strategy-proof and Pareto optimal rule.

The main purpose of the section is to identify those cases where $\varphi$ is a dictatorship, culminating in Theorem 2.3.1.

We start by showing that only extreme points can be boundary outcomes of $\varphi$.

Lemma 2.3.1. Let $p \in A^{N}$ and $\varphi(p) \in \partial A$. Then $\varphi(p) \in C$.

Proof. Let $\varphi(p)=c$. To the contrary suppose that $c \notin C$. Then $c \in$ ]$\widetilde{c}_{L}, \widetilde{c}_{R}\left[\subseteq \partial A\right.$ for some $\widetilde{c}_{L}, \widetilde{c}_{R} \in C$. Let $\ell$ be the line through $c$ perpendicular to $] \widetilde{c}_{L}, \widetilde{c}_{R}[$. Line $\ell$ divides $A$ in a left part and a right part, which we denote by $\mathcal{L}$ and $\mathcal{R}$ respectively, more precisely, $\mathcal{L}=H^{\circ}\left(\ell, \widetilde{c}_{L}\right) \cap A$ and $\mathcal{R}=H^{\circ}\left(\ell, \widetilde{c}_{R}\right) \cap A$. For $x$ in $\partial A \cap \mathcal{L}$ consider the perpendicular bisector $\ell_{x, c}$ of $[x, c]$. If $x \notin\left[\widetilde{c}_{L}, c\right]$, let $d_{x}$ be the point of intersection of $\ell_{x, c}$ and $\partial A$ which is not in $H^{\circ}\left(\ell(x, c), \widetilde{c}_{L}\right)$ (i.e., not in the open half-plane bounded by the straight line through $x$ and $c$ and containing the point $\widetilde{c}_{L}$ ); if $x \in\left[\widetilde{c}_{L}, c\right]$, let $d_{x}$ be the point of intersection of $\ell_{x, c}$ and $\partial A$ which is not in $\left[\widetilde{c}_{L}, c\right]$.

Consider the set $Z$ of points $z \in C \cap \mathcal{L}$ such that $d_{z}$ is in between $d_{z^{\prime}}$ and $c$ for all $z^{\prime} \in C \cap \mathcal{L}$ on the path along the boundary of $A$ passing $\widetilde{c}_{L}$ before $c$. If $Z$ consists of a unique point then call this point $c_{L}$; otherwise, let $c_{L}$ be the point of $Z$ such that $z$ is in between $c_{L}$ and $c$ for all other points $z \in Z .^{2}$ We write $d_{L}$ instead of $d_{c_{L}}$. Clearly, $d_{L} \in \partial A \cap \mathcal{L}$ since $d_{\widetilde{c}_{L}} \in \partial A \cap \mathcal{L}$. Now the choice of $c_{L}$ implies that $d_{L} \in H\left(\ell_{z, c}, z\right)$ for all $z \in C \cap \mathcal{L}$, hence that $\left\|d_{L}-z\right\| \leq\left\|d_{L}-c\right\|$ for all $z \in C \cap \mathcal{L}$. In turn, this implies $\left\|d_{L}-x\right\| \leq\left\|d_{L}-c\right\|$ for all $x \in \overline{\mathcal{L}}$.

Let $S_{L}=\left\{i \in N: p(i) \in H^{\circ}\left(\ell_{c_{L}, c}, c_{L}\right)\right\}$. Since $\left\|d_{L}-\widetilde{c}_{L}\right\| \leq\left\|d_{L}-c\right\|$ and $\ell_{\widetilde{c}_{L}, c} \cap A \subseteq \mathcal{L}$ it follows that $\ell_{c_{L}, c} \cap A \subseteq \mathcal{L}$. Pareto optimality of $\varphi$ implies that either $S_{L}$ is non-empty or $p(i) \in \ell_{c_{L}, c} \cap A$ for all $i \in N$ (since otherwise

\footnotetext{
${ }^{2}$ For a polytope of which the boundary is a regular polygon one can show that $c_{L}=\widetilde{c}_{L}$.
} 
$c_{L}$ would Pareto dominate $\left.c=\varphi(p)\right)$. Now similarly we can define $c_{R}, d_{R}$ and $S_{R}$ for the right part of $A$ and have by Pareto optimality that either $S_{R}$ is non-empty or $p(i) \in \ell_{c_{R}, c} \cap A$ for all $i \in N$. Since $\mathcal{L} \cap \mathcal{R}=\emptyset$ we have that $\ell_{c_{L}, c} \cap A$ and $\ell_{c_{R}, c} \cap A$ are disjoint. This implies that both $S_{L}$ and $S_{R}$ are non-empty.

Now consider the profile $q_{L}$ with $q_{L}(i)=p(i)$ for all $i \notin S_{L}$ and $q_{L}(i)=d_{L}$ for all $i \in S_{L}$. With the aid of the following claim we will prove that $\varphi\left(q_{L}\right)=c_{L}$.

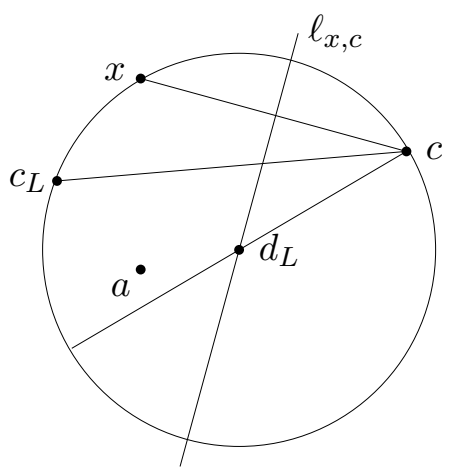

(a)

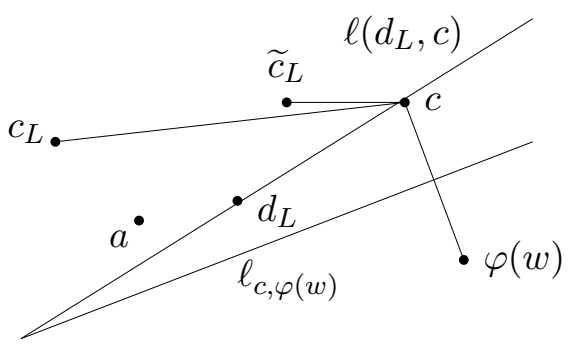

(b)

Figure 2.1: Illustrating the proof of Lemma 2.3.1

Claim Let $v, w \in A^{N}, i \in S_{L}$, and $a \in H^{\circ}\left(\ell_{c_{L}, c}, c_{L}\right)$ with $v(i)=a, w(i)=$ $d_{L}, v(j)=w(j)$ for all $j \in N \backslash\{i\}$, and $\varphi(v) \in\left[d_{L} ; c_{L} \frown c\right]$. Then $\varphi(w) \in$ $\left[d_{L} ; c_{L} \frown c\right]$.

To prove this, first note that $\|a-x\| \leq\|a-c\|$ for all $x \in\left[d_{L} ; c_{L} \frown c\right]$; this is so since $a \in H\left(\ell\left(c, d_{L}\right), c_{L}\right)$ because $c, d_{L} \in \partial A$, and therefore $a \in$ $H\left(\ell_{x, c}, x\right)$ for all $x \in\left[d_{L} ; c_{L} \frown c\right]$. (See Fig. 2.1(a).) Hence SP of $\varphi$ implies $\|a-c\| \geq\|a-\varphi(w)\|$ otherwise agent $i$ could manipulate from $v$ to $w$. Again by strategy-proofness we have $\left\|d_{L}-\varphi(w)\right\| \geq\left\|d_{L}-c\right\|$ otherwise agent $i$ could manipulate from $w$ to $v$. Suppose that $\left\|d_{L}-\varphi(w)\right\|>\left\|d_{L}-c\right\|$. Then, since $c_{L} \in Z$, we have $\varphi(w) \in \mathcal{R}$. Since $d_{L}$ and $c$ are both in $H\left(\ell_{c, \varphi(w)}, c\right)$ we have $A \cap H\left(\ell\left(d_{L}, c\right), \widetilde{c}_{L}\right) \subseteq H\left(\ell_{c, \varphi(w)}, c\right)$. Since $a \in A \cap H^{\circ}\left(\ell\left(d_{L}, c\right), \widetilde{c}_{L}\right)$ it follows that $a \in H^{\circ}\left(\ell_{c, \varphi(w)}, c\right)$, contradicting $\|a-c\| \geq\|a-\varphi(w)\|$. (See Fig. 2.1(b).) Therefore we must have $\left\|d_{L}-\varphi(w)\right\|=\left\|d_{L}-c\right\|$. 
Now the two circles $\odot\left(d_{L},\left\|d_{L}-c\right\|\right)$ and $\odot(a,\|a-c\|)$ intersect in $c$ and say $c^{\prime}$. Since $\|a-c\| \geq\|a-\varphi(w)\|$ and $\left\|d_{L}-\varphi(w)\right\|=\left\|d_{L}-c\right\|$ it follows that $\varphi(w) \in\left[d_{L} ; c^{\prime} \cap c\right]$. But by the choice of $c_{L}$ we have $\left[d_{L} ; c^{\prime} \cap c\right] \cap A=$ $\left[d_{L} ; c_{L} \frown c\right] \cap A$. Therefore $\varphi(w) \in\left[d_{L} ; c_{L} \frown c\right]$, which completes the proof of the Claim.

Repeated application of the Claim yields that $\varphi\left(q_{L}\right) \in\left[d_{L} ; c_{L} \frown c\right]$. For all $x \in\left[d_{L} ; c_{L} \frown c\right]$ we have by construction that $H\left(\ell_{c_{L}, x}, c_{L}\right) \cap A$ is a subset of $H\left(\ell_{c_{L}, c}, c_{L}\right) \cap A$ and therefore contains no dips of the profile $q_{L}$ other than $d_{L}$. Since $S_{R} \neq \emptyset$, it follows that for profile $q_{L}$ the point $c_{L}$ Pareto dominates dominates all $x \in\left[d_{L} ; c_{L} \frown c\right]$ with $x \neq c_{L}$. Hence, $\varphi\left(q_{L}\right)=$ $c_{L}$.

Next consider the profile $r$ with $r(i)=d_{R}$ for all $i \in S_{R}$ and $r(i)=q_{L}(i)$ for all $i \notin S_{R}$. SP of $\varphi$ implies that $\left\|d_{R}-\varphi(r)\right\| \geq\left\|d_{R}-c_{L}\right\|$. But clearly $d_{R} \in H^{\circ}\left(\ell_{c_{L}, c}, c\right)$ and therefore $\left\|d_{R}-c\right\|<\left\|d_{R}-c_{L}\right\|$. Hence, $\left\|d_{R}-\varphi(r)\right\|>\left\|d_{R}-c\right\|$ and by the the definition of $d_{R}$ (similar to $d_{L}$ above) $\left\|d_{R}-c\right\| \geq\left\|d_{R}-x\right\|$ for all $x \in \overline{\mathcal{R}}$. This implies $\varphi(r) \in \mathcal{L}$. By a completely analogous argument, now starting on the right part of $A$ with a profile $q_{R}$, we can show that $\varphi(r) \in \mathcal{R}$. Since $\mathcal{L} \cap \mathcal{R}=\emptyset$, we have a contradiction, which completes the proof of the lemma.

The next lemma follows by combining Lemmas 2.2.1 and 2.3.1.

Lemma 2.3.2. Let $a, b \in A$ and $S \subseteq N$. Then $\varphi\left(a^{S}, b^{N \backslash S}\right) \in C$.

For a subset $B$ of $A$ let $A_{B}$ denote the set of dips for which $B$ is exactly the set of best alternatives, i.e.,

$$
A_{B}=\{x \in A: \mathfrak{b}(x)=B\}
$$

For a singleton $\{a\}$ we write $A_{a}$ instead of $A_{\{a\}}$. We can make the following observations. If $B \subseteq A$ and $B \nsubseteq C$, then $A_{B}=\emptyset$. If $B=\{a, b\}$ for some distinct $a, b \in C$ then $A_{B} \subseteq \ell_{a, b}$ and if $B=\{a, b, c\}$ for some distinct $a, b, c \in C$ then $A_{B} \subseteq \ell_{a, b} \cap \ell_{b, c}$. Moreover, if $A_{a} \neq \emptyset$ for some $a \in C$ then $A_{a}$ is convex. This can be seen as follows. Let $x, y \in A_{a}$ and let $b \in A \backslash\{a\}$, then $x, y \in H^{\circ}\left(\ell_{a, b}, b\right)$, hence $z \in H^{\circ}\left(\ell_{a, b}, b\right)$ for any convex combination $z$ of $x$ and $y$, so $b \notin \mathfrak{b}(z)$. Since $b$ was arbitrary and $\mathfrak{b}(z) \neq \emptyset$, this implies 
that $z \in A_{a}$. Let

$$
\mathcal{B}=\left\{a \in A: A_{a} \neq \emptyset\right\} .
$$

Elements of $\mathcal{B}$ are called single-best alternatives. Combining our observations we see that for each $a \in \mathcal{B}$ the set $A_{a}$ is a convex polygon which is open relative to $A$, and that $A=\cup_{x \in \mathcal{B}} \bar{A}_{x}$.

The following two lemmas establish further facts about alternatives in $\mathcal{B}$.

Lemma 2.3.3. Let $a, b$, and $c$ be three distinct alternatives in $\mathcal{B}$. Then $m_{a, b, c}$ exists and $m_{a, b, c} \in A^{\circ}$.

Proof. Since $a, b, c \in C$ they are not collinear and thus $m_{a, b, c}$ exists. The straight lines $\ell_{a, b}, \ell_{a, c}$, and $\ell_{b, c}$ intersect at $m_{a, b, c}$ and determine six open non-empty regions which divide $A^{\circ} \backslash\left(\ell_{a, b} \cup \ell_{a, c} \cup \ell_{b, c}\right)$ into disjoint sets. A single dipped preference $R_{x}$ is constant over $a, b$, and $c$ as long as $x$ stays in one of these regions. Call these regions $V_{a b c}, V_{b a c}, V_{b c a}, V_{c b a}, V_{c a b}$ and $V_{a c b}$; for $x \in V_{a b c}$ we have that $a$ is (strictly) preferred over $b$ and $b$ over $c$ according to $R_{x}$, and similarly for the other five regions. Now $A_{a} \subseteq\left(V_{a b c} \cup\right.$ $\left.V_{a c b} \cup \ell_{b, c}\right)^{\circ} \cap A$. Since $A_{a}$ is non-empty we have that $\left(V_{a b c} \cup V_{a c b} \cup \ell_{b, c}\right)^{\circ} \cap A$ is non-empty and therefore $\left(V_{a b c} \cup V_{a c b} \cup \ell_{b, c}\right)^{\circ} \cap A^{\circ}$ is non-empty. Similarly $\left(V_{b c a} \cup V_{b a c} \cup \ell_{a, c}\right)^{\circ} \cap A^{\circ}$ and $\left(V_{c a b} \cup V_{c b a} \cup \ell_{a, b}\right)^{\circ} \cap A^{\circ}$ are non-empty. Take points $x_{a}, x_{b}, x_{c}$ in these respective regions, then $m_{a, b, c}$ is in the convex hull of these three points and is therefore an interior point of $A$.

Lemma 2.3.4. Let $a \in \mathcal{B}$. Then $A^{\circ} \backslash \bar{A}_{a}$ is connected.

Proof. If $A^{\circ} \backslash \bar{A}_{a}=A_{b}$ for some $b \in \mathcal{B}$ we are done because $A_{b}$ is obviously connected as it is convex. Therefore let $y, z \in A^{\circ}$ with $y \in A_{b}$ and $z \in A_{c}$ for some distinct $b, c \in \mathcal{B} \backslash\{a\}$. Since $A^{\circ} \subseteq \cup\left\{\bar{A}_{x}: x \in \mathcal{B}\right\}$ it is sufficient to prove that there is a path in $A^{\circ} \backslash \bar{A}_{a}$ connecting $y$ and $z \cdot{ }^{3}$ Lemma 2.3.3 implies that $m_{a, b, c} \in A^{\circ}$. Convexity of $A$ implies that $A^{\circ}$ is convex. Hence, $\left[y, m_{a, b, c}\right] \subseteq A^{\circ}$ and $\left[z, m_{a, b, c}\right] \subseteq A^{\circ}$. With notations as in the proof of Lemma 2.3.3 we may further conclude that $\left[y, m_{a, b, c}\left[\subseteq\left(V_{b c a} \cup V_{b a c} \cup \ell_{a, c}\right)^{\circ}\right.\right.$ and $\left[z, m_{a, b, c}\left[\subseteq\left(V_{c a b} \cup V_{c b a} \cup \ell_{a, b}\right)^{\circ}\right.\right.$. Since $A_{a} \subseteq\left(V_{a b c} \cup V_{a c b} \cup \ell_{b, c}\right)^{\circ} \cap A$ and $\left(V_{a b c} \cup V_{a c b} \cup \ell_{b, c}\right)^{\circ}$ is disjoint from both $\left(V_{b c a} \cup V_{b a c} \cup \ell_{a, c}\right)^{\circ}$ and $\left(V_{c a b} \cup\right.$

\footnotetext{
${ }^{3}$ I.e. the graph of a continuous function $[0,1] \rightarrow A$ with $0 \mapsto y$ and $1 \mapsto z$.
} 
$\left.V_{c b a} \cup \ell_{a, b}\right)^{\circ}$ it follows that we can construct a path from $y$ to $z$ in $A^{\circ} \backslash \bar{A}_{a}$, as follows. Choose points $y^{\prime} \in\left[y, m_{a, b, c}\left[\right.\right.$ and $\left.\left.z^{\prime} \in\right] m_{a, b, c}, z\right]$ close enough to $m_{a, b, c}$ such that there is a path $P$ from $y^{\prime}$ to $z^{\prime}$ in $A^{\circ}$ disjoint from $\bar{A}_{a}$; then the desired path is $\left[y, y^{\prime}\right] \cup P \cup\left[z^{\prime}, z\right]$. (See Fig. 2.2.)

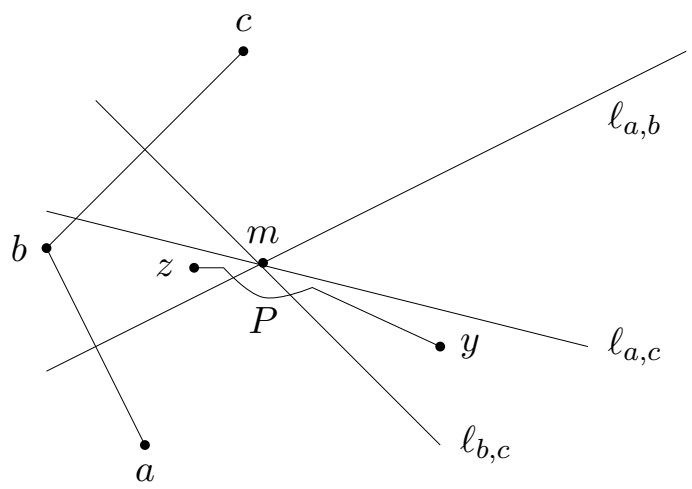

Figure 2.2: Illustrating the proof of Lemma 2.3.4: path between $y$ and $z ; m=$ $m_{a, b, c}$

In what follows we often use a notation like $R=\ldots x \ldots y z \ldots(x, y, z \in C)$ to express that, in the preference $R, x$ is strictly preferred to $y$, and $y$ to $z$ such that other alternatives in $C$ are either above $y$ or below $z$ in preference. The following lemma is a simple consequence of (intermediate) strategy-proofness. We leave its proof to the reader.

Lemma 2.3.5. Let $\emptyset \neq S \subseteq N, p, q \in A^{N}$ and $x, y \in C$ with $x \neq y$ such that $(i) p(j)=q(j)$ for all $j \in N \backslash S$; (ii) $p(i)=p(j)$ and $q(i)=q(j)$ for all $i, j \in S$; (iii) for all $i \in S, p(i)$ and $q(i)$ order the alternatives of $C$ strictly and coincide on $C$ except for a swap between $x$ and $y: p(i)=\ldots x y \ldots$, $q(i)=\ldots y x \ldots ;$ and $(i v) \varphi(p), \varphi(q) \in C, \varphi(p) \neq \varphi(q)$. Then $x=\varphi(p)$ and $y=\varphi(q)$.

Lemma 2.3.5 says that if an agent's preference changes by swapping two alternatives in $C$ while not changing the (strict) order between other alternatives in $C$, and if therefore the alternative assigned by the rule changes, then the two swapped alternatives must be the alternatives assigned by the rule. $^{4}$

\footnotetext{
${ }^{4}$ This is a restricted version of (Maskin) monotonicity, which is implied by strategy-
} 
The next result can be interpreted as saying that $\varphi$ leaves no room for compromising at two-dip profiles.

Lemma 2.3.6. Let $S \subseteq N, a, b \in \mathcal{B}, x \in A_{a}, y \in A_{b}$, and $p=\left(x^{S}, y^{N \backslash S}\right) \in$ $A^{N}$. Then $\varphi(p) \in\{a, b\}$.

Proof. If $a=b$ then the claim in the lemma follows from Pareto-optimality. Thus, let $a \neq b$ and suppose that $\varphi(p)=c \notin\{a, b\}$. We derive a contradiction. Pareto optimality implies $R_{x}=a \ldots c \ldots b \ldots$ and $R_{y}=b \ldots c \ldots a \ldots$ We take a path from $x$ to $c$ with the following properties (see below for a formal description): (i) along this path, $c$ can only become worse compared to other elements of $C$; (ii) along this path the preference over elements of $C$ changes by swaps of at most two alternatives at the same time; and (iii) along this path preferences over $C$ are strict except at swaps as in (ii). Formally, this path is the graph of a continuous function $\pi$ from $[0,1]$ to $A$ with $\pi(0)=x$ and $\pi(1)=c$ such that (i) for all $t_{1}<t_{2}$ and $z \in C$, if $(z, c) \in R_{\pi\left(t_{1}\right)}$ then $(z, c) \in R_{\pi\left(t_{2}\right)}$ and $(c, z) \notin R_{\pi\left(t_{2}\right)}$; (ii) for all $t \in[0,1]$ there are no distinct $z, z^{\prime}, z^{\prime \prime} \in C$ such that $\pi(t) \in \ell_{z, z^{\prime}} \cap \ell_{z, z^{\prime \prime}}$; and (iii) there are at most finitely many $t$ for which $\pi(t) \in \ell_{z, z^{\prime}}$ for some $z, z^{\prime} \in C$. We call a pair $R_{\pi\left(t_{1}\right)}, R_{\pi\left(t_{2}\right)}$ an 'elementary change' if the latter preference arises from the former after swapping two adjacent alternatives in $C$.

Let $p^{t}=\left(\pi(t)^{S}, y^{N \backslash S}\right)$. Then $\varphi\left(p^{1}\right) \neq c$ since $c$ is not Pareto optimal at $p^{1}$. Thus, there must be a first elementary change such that $\varphi\left(p^{t_{1}}\right)=c$ and $\varphi\left(p^{t_{2}}\right)=d \neq c$. By Lemma 2.3.5 this implies that $R_{\pi\left(t_{1}\right)}=\ldots c d \ldots$ whereas $R_{\pi\left(t_{2}\right)}=\ldots d c \ldots$

Now there are two cases.

Case $1 R_{y}=b \ldots d \ldots c \ldots a \ldots$

Then in the same way as above we can find a path $\rho$ from $y$ to $c$, profiles $q^{t}=\left(\pi\left(t_{1}\right)^{S}, \rho(t)^{N \backslash S}\right)$ and $t_{3}$ and $t_{4}$ such that $\varphi\left(q^{t_{3}}\right)=c, \varphi\left(q^{t_{4}}\right)=e \neq c$ and $R_{\rho\left(t_{3}\right)}$ and $R_{\rho\left(t_{4}\right)}$ form an elementary change swapping $c$ and $e$. Consider $r=$ $\left(\pi\left(t_{2}\right)^{S}, \rho\left(t_{4}\right)^{N \backslash S}\right)$ and $u^{t}=\left(\pi(t)^{S}, \rho\left(t_{3}\right)^{N \backslash S}\right)$ for $t \in\left[t_{1}, t_{2}\right]$. So, $\varphi\left(u^{t_{1}}\right)=$ $\varphi\left(q^{t_{3}}\right)=c$. Let $\varphi(r)=f$. Note that $R_{\rho\left(t_{3}\right)}=\ldots d \ldots c e \ldots$ and $R_{\rho\left(t_{4}\right)}=$

proofness. 
...d...ec.... Since $\varphi\left(u^{t_{1}}\right)=c$ it follows by Pareto optimality and Lemma 2.3.5 that $\varphi\left(u^{t_{2}}\right)=d$. Summarizing we have:

\begin{tabular}{|c|c|c|}
\hline \hline & $u^{t_{1}}=q^{t_{3}}$ & $q^{t_{4}}$ \\
\hline$i \in S$ & $u^{t_{1}}(i)=R_{\pi\left(t_{1}\right)}=\ldots c d \ldots$ & $q^{t_{4}}(i)=R_{\pi\left(t_{1}\right)}=\ldots c d \ldots$ \\
$i \in N \backslash S$ & $u^{t_{1}}(i)=R_{\rho\left(t_{3}\right)}=\ldots d \ldots c e \ldots$ & $q^{t_{4}}(i)=R_{\rho\left(t_{4}\right)}=\ldots d \ldots e c \ldots$ \\
\hline & $\varphi\left(u^{t_{1}}\right)=c$ & $\varphi\left(q^{t_{4}}\right)=e$ \\
\hline \hline & $u^{t_{2}}$ & $r$ \\
\hline$i \in S$ & $u^{t_{2}}(i)=R_{\pi\left(t_{2}\right)}=\ldots d c \ldots$ & $r(i)=R_{\pi\left(t_{2}\right)}=\ldots d c \ldots$ \\
$i \in N \backslash S$ & $u^{t_{2}}(i)=R_{\rho\left(t_{3}\right)}=\ldots d \ldots c e \ldots$ & $r(i)=R_{\rho\left(t_{4}\right)}=\ldots d \ldots e c \ldots$ \\
\hline & $\varphi\left(u^{t_{2}}\right)=d$ & $\varphi(r)=f$ \\
\hline \hline
\end{tabular}

There are two subcases.

Subcase $d \neq f$. Comparing $u^{t_{2}}$ and $r$, Lemma 2.3 .5 implies $R_{\rho\left(t_{3}\right)}=$ $\ldots d f \ldots c e \ldots$ and $R_{\rho\left(t_{4}\right)}=\ldots f d \ldots e c \ldots$ So $c, d, e$, and $f$ are all different. Comparing $q^{t_{4}}$ and $r$, Lemma 2.3 .5 implies (i) $R_{\pi\left(t_{1}\right)}=\ldots e f \ldots c d \ldots$ and $R_{\pi\left(t_{2}\right)}=\ldots f e \ldots d c \ldots$ or (ii) $R_{\pi\left(t_{1}\right)}=\ldots c d \ldots e f \ldots$ and $R_{\pi\left(t_{2}\right)}=\ldots d c \ldots f e \ldots$ But (i) implies that $\varphi\left(u^{t_{1}}\right)=c$ is Pareto dominated by $f$ at $u^{t_{1}}$, a contradiction; and (ii) implies that $\varphi\left(q^{t_{4}}\right)=e$ is Pareto dominated by $d$ at $q^{t_{4}}$, likewise a contradiction. So this subcase cannot occur.

Subcase $d=f$. Comparing $q^{t_{4}}$ and $r$, Lemma 2.3.5 implies $R_{\pi\left(t_{1}\right)}=\ldots e d \ldots$ and $R_{\pi\left(t_{2}\right)}=\ldots d e \ldots$ This implies the contradiction $c=e$ and therefore also this subcase cannot occur. This ends the proof of Case 1 .

Case $2 R_{y}=b \ldots c \ldots d \ldots a \ldots$

By interchanging the roles of $c$ and $d$ and thus those of $R_{\pi\left(t_{1}\right)}$ and $R_{\pi\left(t_{2}\right)}$, and then proceeding like in Case 1, we derive a similar contradiction.

Call a coalition $S \subseteq N$ quasi-decisive (given $\varphi$ ) if $\varphi(p)=a$ for all $a, b \in \mathcal{B}$ and every profile $p=\left(x^{S}, y^{N \backslash S}\right)$ with $x \in A_{a}$ and $y \in A_{b}$.

Lemma 2.3.7. Let $|\mathcal{B}| \geq 3$. Then for every $S \subseteq N$, either $S$ or $N \backslash S$ is quasi-decisive. 
Proof. Let $S \subseteq N$. If $S=N$ or $S=\emptyset$ then we are done by Pareto optimality. So assume $S \neq \emptyset, N$. Clearly, $S$ and $N \backslash S$ cannot both be quasi-decisive. Let $a, b$ be two different elements of $\mathcal{B}$, and let $x \in A_{a}$, $y \in A_{b}$, and $p=\left(x^{S}, y^{N \backslash S}\right)$. By Lemma 2.3.6, $\varphi(p) \in\{a, b\}$. Without loss of generality assume that $\varphi(p)=a$. Take $c, d \in \mathcal{B}, v \in A_{c}$ and $w \in A_{d}$ arbitrary, and let $q=\left(v^{S}, w^{N \backslash S}\right)$. It is sufficient to prove that $\varphi(q)=c$. By Pareto optimality we may assume that $c \neq d$. Lemma 2.3.6 implies $\varphi(q) \in\{c, d\}$. We proceed in four steps.

Step 1 Assume $v=x$.

Then, since $v=x$ we have $c=a$. If also $b=d$, then $\varphi(q)=c=a$ otherwise coalition $N \backslash S$ could manipulate from $p$ to $q$ and obtain $d=b$. So assume $b \neq d$. By Lemma 2.3.4 there is a path from $y$ to $w$ disjoint from $\bar{A}_{a}$. Along this path we can find points $y=u_{1}, u_{2}, \ldots, u_{k}=w(k \geq 2)$ such that $u_{t} \in A_{f_{t}}, f_{t} \in \mathcal{B}$, for $t=1, \ldots, k$ (so $f_{1}=b, f_{k}=d$ ) and such that for every $t=1, \ldots, k-1$ we have $R_{u_{t \mid \mathcal{B}}}=f_{t} f_{t+1} \ldots$ and $R_{u_{t+1 \mid \mathcal{B}}}=f_{t+1} f_{t} \ldots{ }^{5}$ For every $t=1, \ldots, k$ let $p^{t}=\left(x^{S}, u_{t}^{N \backslash S}\right)$. Thus, $p^{1}=p$ and $p^{k}=q$. Since $\varphi\left(p^{1}\right)=a$, $f_{1}, f_{2} \neq a, R_{u_{2} \mid \mathcal{B}}=f_{2} f_{1} \ldots$, and $\varphi\left(p^{2}\right) \in\left\{a, f_{2}\right\}$ by Lemma 2.3.6, we have by strategy-proofness that $\varphi\left(p^{2}\right)=a$, since otherwise coalition $N \backslash S$ could manipulate from $p^{1}=p$ to $p^{2}$, obtaining $f_{2}$ instead of $a$. Similarly, it follows from this that $\varphi\left(p^{3}\right)=a$ and so on, so that $a=\varphi\left(p^{k}\right)=\varphi(q)$.

Step 2 Assume $v \neq x$ and $d \neq a$.

Consider $p^{\prime}=\left(x^{S}, w^{N \backslash S}\right)$. Then Step 1 implies $\varphi\left(p^{\prime}\right)=a$. By Step 1 applied to $p^{\prime}$ and $q$, if $\varphi(q)=d$ then $\varphi\left(p^{\prime}\right)=d$, a contradiction since $d \neq a$. Hence $\varphi(q)=c$.

Step 3 Assume $y=v, w=x$.

Let $e \in \mathcal{B} \backslash\{a, b\}$ and $u \in A_{e}$. Then $\varphi(p)=\varphi\left(x^{S}, y^{N \backslash S}\right)=a$ implies by Step 1 that $\varphi\left(x^{S}, u^{N \backslash S}\right)=a$. In turn, by Step 2 this implies that $\varphi\left(y^{S}, u^{N \backslash S}\right)=b$, which by Step 1 again yields $\varphi(q)=\varphi\left(y^{S}, x^{N \backslash S}\right)=b$. Since $y=v$, we have $b=c$, so $\varphi(q)=c$.

\footnotetext{
${ }^{5} R_{x \mid D}$ denotes the restriction of preference $R_{x}$ to $D \subseteq A$.
} 
Step 4 Assume $v \neq x, d=a$.

Then $\varphi(p)=\varphi\left(x^{S}, y^{N \backslash S}\right)=a$ implies by Step 3 that $\varphi\left(y^{S}, x^{N \backslash S}\right)=b$, hence by Step 1 we obtain $\varphi\left(y^{S}, w^{N \backslash S}\right)=b$. If $y=v$, hence $b=c$, we have $\varphi(q)=\varphi\left(v^{S}, w^{N \backslash S}\right)=\varphi\left(y^{S}, w^{N \backslash S}\right)=b=c$. If $y \neq v$ then $d=a \neq b$ and Step 2 imply $\varphi(q)=\varphi\left(v^{S}, w^{N \backslash S}\right)=c$.

Since the case $v=x$ is covered in Step 1 , the case $v \neq x$ and $d \neq a$ in Step 2, and the case $v \neq x$ and $d=a$ in Step 4, all cases are covered, so the proof is complete.

Lemma 2.3.8. Let $|\mathcal{B}| \geq 3$. Then for every $S \subseteq N$, either $S$ or $N \backslash S$ is decisive.

Proof. Let $S \subseteq N$. Clearly, $S$ and $N \backslash S$ cannot both be decisive. By Lemma 2.3.7 either $S$ or $N \backslash S$ is quasi-decisive. Assume that $S$ is quasidecisive, the other case is analogous. Consider a profile $p$ with $p(i)=x$ for all $i \in S$ such that $x \in A_{a}$ for some $a \in \mathcal{B}$. We first prove that $\varphi(p)=a$. Take a $d \in \mathcal{B}$ such that $a \in \partial A_{d}$, and take a $v \in A_{d}$. Let $r=\left(x^{S}, v^{N \backslash S}\right)$. Quasi-decisiveness of $S$ implies that $\varphi(r)=a$. By applying intermediate strategy-proofness to the profiles $r$ and $p$ we obtain $\|v-a\| \geq\|v-\varphi(p)\|$. Since we can take $v$ as close to $a$ as desired, this implies $\varphi(p)=a$. Finally, let $q$ be a profile with $q(i)=y \in A$ for all $i \in S$, and suppose that $\varphi(q) \notin$ $\mathfrak{b}(y)$. Then take $b \in \mathfrak{b}(y) \cap \mathcal{B}$ (this is possible since $A=\cup\left\{\bar{A}_{x}: x \in \mathcal{B}\right\}$ ) and $z \in A$ such that $z \in A_{b}$. By the first part of the proof, $\varphi\left(q^{\prime}\right)=b$ where $q^{\prime}(i)=z$ for all $i \in S$ and $q^{\prime}(i)=q(i)$ for all $i \in N \backslash S$. Then $S$ can manipulate from $q$ to $q^{\prime}$, violating (intermediate) SP.

In what follows we pin down those polytopes $A$ for which the intersection of two decisive sets is again decisive. Call a finite subset of $\mathbb{R}^{2}$ rectangular if it consists of exactly four points which are the vertices of one and the same rectangle.

Lemma 2.3.9. Let the following conditions hold:

(i) $|\mathcal{B}| \geq 3$.

(ii) There are distinct $a, b, c \in \mathcal{B}$ such that $m_{a, b, c} \in \partial A_{a} \cap A^{\circ}$ and such that there is no $x \in \mathcal{B}$ for which $\{a, b, c, x\}$ is rectangular. 
Let $S, T \subseteq N$ be both decisive. Then $S \cap T$ is decisive.

Proof. Suppose, to the contrary (cf. Lemma 2.3.8), that $N \backslash(S \cap T)$ is decisive. Then we have a partition $X=S \cap T, Y=S \backslash T$ and $Z=N \backslash S$ of $N$ such that $X, Y$ and $Z$ are not decisive. Hence any union of two of these sets, being the complement of the third one, is decisive. We will derive a contradiction.

Let $a, b$ and $c$ be as in (ii) in the statement of the lemma. We choose $\widetilde{a} \in A^{\circ}$ close to $a$ and $\bar{b}, \bar{c}, \bar{a}_{b}, \bar{a}_{c} \in A^{\circ}$ close to $m:=m_{a, b, c}$ (how close will be specified below) with

$$
\begin{aligned}
R_{\bar{b}} & =b a \ldots c \ldots \\
R_{\bar{c}} & =c a \ldots b \ldots \\
R_{\bar{a}_{b}} & =a b \ldots c \ldots \\
R_{\bar{a}_{c}} & =a c \ldots b \ldots \\
R_{\widetilde{a}} & =\ldots c \ldots b \ldots a
\end{aligned}
$$

and such that all these preferences (restricted to $C$ ) are strict. (This is possible in particular since $m_{a, b, c} \in \partial A_{a} \cap A^{\circ}$. (See Fig. 2.3(a).) If $\widetilde{a}$

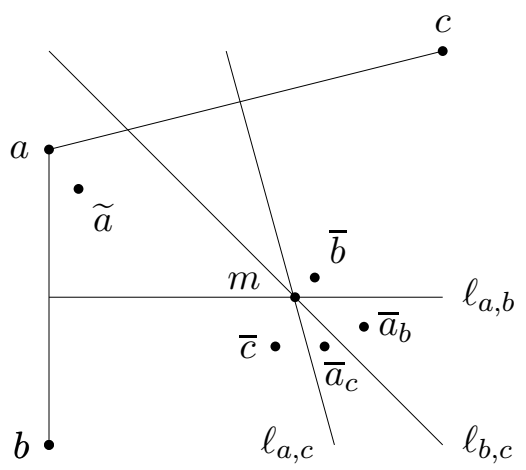

(a)

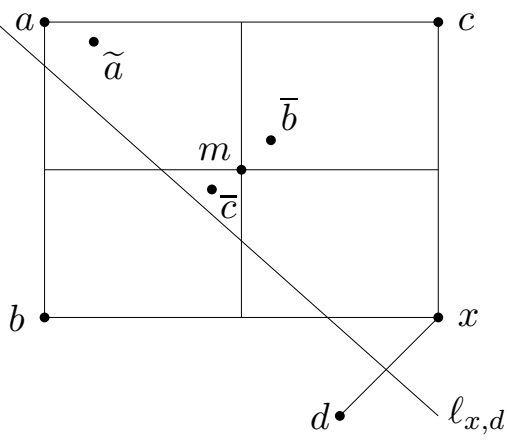

(b)

Figure 2.3: Illustrating the proof of Lemma 2.3.9

cannot be chosen this way then we choose it such that $R_{\widetilde{a}}=\ldots b \ldots c \ldots a$ and proceed analogously.) By choosing these points close enough to $a$ and $m$, respectively, we have that for any profile $q$ with dips at these points, 
any alternative in $A^{\circ}$ is Pareto dominated by a boundary point. Hence, by Lemma 2.3.1, $\varphi(q) \in C$ for such profiles $q$. In particular, $\varphi$ assigns an alternative in $C$ to any of the following profiles:

$$
\begin{aligned}
p & =\left(\widetilde{a}^{X}, \bar{b}^{Y}, \bar{c}^{Z}\right) \\
p^{\bar{a}_{b}} & =\left(\widetilde{a}^{X}, \bar{a}_{b}^{Y}, \bar{c}^{Z}\right) \\
p^{\bar{a}_{c}} & =\left(\widetilde{a}^{X}, \bar{b}^{Y}, \bar{a}_{c}^{Z}\right) \\
r & =\left(\widetilde{a}^{X}, \bar{a}_{b}^{Y}, \bar{a}_{c}^{Z}\right) .
\end{aligned}
$$

Claim $\varphi(p) \in\{a, b, c\}$.

Let $\varphi(p)=x, \varphi\left(p^{\bar{a}_{b}}\right)=y$ and $\varphi\left(p^{\bar{a}_{c}}\right)=z$. Since $Y \cup Z$ is decisive and since $\bar{a}_{b}, \bar{a}_{c} \in A_{a}$, we have by strategy-proofness that $\varphi(r)=a$.

First consider $r$ and $p^{\bar{a}_{b}}$. By Lemma 2.3.5 it follows that the first change, starting from below, in the preferences $R_{\bar{a}_{c}}$ and $R_{\bar{c}}$ must be a swap between $\varphi(r)$ and $\varphi\left(p^{\bar{a}_{b}}\right)$. Since $\varphi(r)=a$, this implies that either (i.a) $y=a$ or (i.b) $y=c$.

Similarly, comparing $r$ and $p^{\bar{a}_{c}}$ yields either (ii.a) $z=a$ or (ii.b) $z=b$.

We next compare $p$ and $p^{\bar{a}_{b}}$. These profiles differ for the agents in $Y$, who have dips $\bar{b}$ in $p$ and dips $\bar{a}_{b}$ in $p^{\bar{a}_{b}}$. By going from $\bar{b}$ to $\bar{a}_{b}$ only the perpendicular bisector $\ell_{a, b}$ is crossed. By Lemma 2.3.5, as before the first change in preference from below must be a swap between $\varphi(p)=x$ and $\varphi\left(p^{\bar{a}_{b}}\right)=y$, but this implies that $\ell_{x, y}$ coincides with $\ell_{a, b}$. This implies that, if $x \neq y$, then $x$ is the mirror image of $y$ with respect to the perpendicular bisector of $[a, b]$. Therefore, we have either (iii.a) $x=y$ or (iii.b) $x \neq y$ and $\sigma_{a, b}(x)=y$.

Similarly, comparing $p$ and $p^{\bar{a}_{c}}$ yields either (iv.a) $x=z$ or (iv.b) $x \neq z$ and $\sigma_{a, c}(x)=z$.

If $x=y$ or $x=z$, then (i.a) and (i.b) or (ii.a) and (ii.b) imply that $x \in\{a, b, c\}$. Let $x \neq y, x \neq z$. Then $\sigma_{a, b}(x)=y$ and $\sigma_{a, c}(x)=z$ by (iii.b) and (iv.b). Since $a, b, c$ are distinct and thus $\ell_{a, b} \neq \ell_{a, c}$ it follows that $z \neq y$. If $y=a$ or $z=a$, then $\sigma_{a, b}(x)=y$ and $\sigma_{a, c}(x)=z$ imply $x=b$ or $x=c$, hence we are done again. So, assume $y \neq a$ and $z \neq a$, then $y=c$ by (i.b) 
and $z=b$ by (ii.b). Then by (iii.b) and (iv.b) we have $\sigma_{a, b}(x)=y=c$ and $\sigma_{a, c}(x)=z=b$. We now assume $a \neq x$ and derive a contradiction, which will complete the proof of the Claim. Since $\sigma_{a, c}(a)=c$ and $\sigma_{a, b}(a)=b$ the assumption $a \neq x$ implies that $a, b, c$ and $x$ are vertices of a rectangle. (See Fig. 2.3(b).) To derive the contradiction it is, in view of condition (ii) in the lemma, sufficient to prove that $x \in \mathcal{B}$. Let $d \in \mathfrak{b}\left(R_{a}\right)$, hence $d \notin\{a, b, c\}$, with $d \neq x$. We will show that $m \in \ell_{x, d}$. Since $m=m_{a, x}$, this implies that $a, x$, and $d$ are on the same circle with center $m$. Since $a$ and $x$ are on the same diameter in this circle we have that $\|d-a\|<\|x-a\|$, contradicting $d \in \mathfrak{b}\left(R_{a}\right)$. So $a \in A_{x}$, hence $x \in \mathcal{B}$.

We are left to show that $m \in \ell_{x, d}$. Suppose, to the contrary, that $m \notin \ell_{x, d}$. Since $d \in \mathfrak{b}\left(R_{a}\right)$ we have $a \in H\left(\ell_{x, d}, x\right)$, hence $m \in H^{\circ}\left(\ell_{x, d}, x\right)$ and therefore $\bar{b}, \bar{c} \in H^{\circ}\left(\ell_{x, d}, x\right)$ by the choice of these points (note that these points can be chosen close enough to $m$ to achieve this, given that there are only finitely many candidates for the points $x$ and $d$ ). By the choice of $\widetilde{a}$, also $\widetilde{a} \in H^{\circ}\left(\ell_{x, d}, x\right)$. But this implies that $d$ Pareto dominates $x$ at profile $p$, contradicting $\varphi(p)=x$. This completes the proof of the Claim.

Consider the profile $p^{\prime}=\left(\bar{c}^{X}, \bar{b}^{Y}, \bar{c}^{Z}\right)$. Since $X \cup Z$ is decisive we have that $\varphi\left(p^{\prime}\right)=c$. Strategy-proofness and the Claim now imply that $x=\varphi(p)=$ $c$.

Next we prove that $\varphi\left(p^{\bar{a}_{c}}\right)=z \in\{a, b\}$. Note that $z \in \mathcal{C}$. Because of $\varphi(r)=a$, strategy-proofness implies that $z$ is weakly preferred to $a$ at $R_{\bar{b}}$. So, $z \in\{a, b\}$.

Let $p^{\prime \prime}=\left(\bar{b}^{X}, \bar{b}^{Y}, \bar{a}_{c}^{Z}\right)$. Since $X \cup Y$ is decisive we have $\varphi\left(p^{\prime \prime}\right)=b$. Then strategy-proofness implies $\varphi\left(p^{\bar{a}_{c}}\right)=z=b$. Since $\varphi(p)=c$, coalition $Z$ can manipulate from $p^{\bar{a}_{c}}$ to $p$, violating strategy-proofness.

Before continuing with the main result of this section a consideration of the polytopes to which the preceding lemmas apply is in order. First, let $|C|=3$, i.e., $A$ is a triangle including its interior. If this triangle is acute then $\mathcal{B}=C$, hence $|\mathcal{B}|=3$ and also condition (ii) of Lemma 2.3.9 is trivially satisfied. If this triangle is not acute, however, then $|\mathcal{B}|=2$ and this case will be studied in the next section. Second, let $|C|>3$. If $|\mathcal{B}|=2$, which 
means, roughly, that the polytope $A$ is rather flat, then again the results in the next section apply. For cases where $|\mathcal{B}| \geq 3$ we provide an exact characterization of those polytopes where condition (ii) of Lemma 2.3.9 is not satisfied. We start with an auxiliary lemma.

Lemma 2.3.10. Let $|\mathcal{B}| \geq 3$ and let $a \in \mathcal{B}$. Then there are distinct $b, c \in$ $\mathcal{B} \backslash\{a\}$ such that $m_{a, b, c} \in A^{\circ} \cap \partial A_{a}$.

Proof. For any two distinct points $x, y \in \mathcal{B} \backslash\{a\}$, we have $m_{a, x, y} \in A^{\circ}$ by Lemma 2.3.3. Now, since $\mathcal{B}$ is finite there must be distinct $b, c \in \mathcal{B} \backslash\{a\}$ such that the set $H^{\circ}\left(\ell_{a, b}, b\right) \cap H^{\circ}\left(\ell_{a, c}, b\right)$ contains no point of the form $m_{a, x, y}$ for distinct $x, y \in \mathcal{B} \backslash\{a\} .{ }^{6}$ We claim that $H^{\circ}\left(\ell_{a, b}, b\right) \cap H^{\circ}\left(\ell_{a, c}, c\right) \cap A$ also has empty intersection with $\ell_{a, x}$ for any $x \in C \backslash \mathcal{B}$. If not, then there is such an $x$ and a point $x_{a} \in H^{\circ}\left(\ell_{a, b}, b\right) \cap H^{\circ}\left(\ell_{a, c}, b\right) \cap H^{\circ}\left(\ell_{a, x}, x\right) \cap A$ with $R_{x_{a}}=a x \ldots$ and such that $R_{\sigma_{a, x}\left(x_{a}\right)}=x a \ldots$ But then $\sigma_{a, x}\left(x_{a}\right) \in A_{x}$, so $x \in \mathcal{B}$, a contradiction. It follows that $H^{\circ}\left(\ell_{a, b}, b\right) \cap H^{\circ}\left(\ell_{a, c}, b\right) \cap A \subseteq A_{a}$. Since $m_{a, b, c}=\ell_{a, b} \cap \ell_{a, c}$ this implies that $m_{a, b, c} \in \bar{A}_{a}$. Since, clearly, $m_{a, b, c} \notin A_{a}$ we have $m_{a, b, c} \in \partial A_{a}$.

The next lemma characterizes those polytopes where condition (ii) of Lemma 2.3.9 is not satisfied.

Lemma 2.3.11. Let $|\mathcal{B}| \geq 3$. Then condition (ii) in Lemma 2.3.9 does not hold if and only if $\mathcal{B}$ is rectangular.

Proof. For the only-if direction, assume that condition (ii) in Lemma 2.3.9 does not hold. We show that $\mathcal{B}$ is rectangular. The proof of this fact proceeds in a few steps. We start with stating (without proof) the following useful fact.

Step 1 A set $Q \subseteq \mathbb{R}^{2}$ is an orthant if it is of the form $Q=\left\{p+\lambda_{1} x^{1}+\lambda_{2} x^{2} \mid\right.$ $\left.\lambda_{1}, \lambda_{2} \geq 0\right\}$ for some $p \in \mathbb{R}^{2}$ and some perpendicular vectors $x^{1}, x^{2} \in \mathbb{R}^{2} \backslash$ $\{0\}$. Denote $p(Q)=p$. Let $X$ be a compact and convex subset of $\mathbb{R}^{2}$ with non-empty interior, and suppose $X$ can be written as $X=\bigcup_{i \in I}\left(Q^{i} \cap X\right)$ where $I$ is some index set, $Q^{i}$ is an orthant with $p\left(Q^{i}\right) \in X^{\circ}$ for each $i \in I$, and $\left(Q^{j} \cap X\right)^{\circ} \cap\left(Q^{k} \cap X\right)^{\circ}=\emptyset$ for all distinct $j, k \in I$. Then $|I|=4$.

\footnotetext{
${ }^{6}$ Start with arbitrary $x, y$. If there is a point $m_{a, x^{\prime}, y^{\prime}} \in H^{\circ}\left(\ell_{a, x}, x\right) \cap H^{\circ}\left(\ell_{a, y}, y\right)$, then $m_{a, x, y} \notin H^{\circ}\left(\ell_{a, x^{\prime}}, x^{\prime}\right) \cap H^{\circ}\left(\ell_{a, y^{\prime}}, y^{\prime}\right)$ and we continue with $x^{\prime}, y^{\prime}$, and so on.
} 


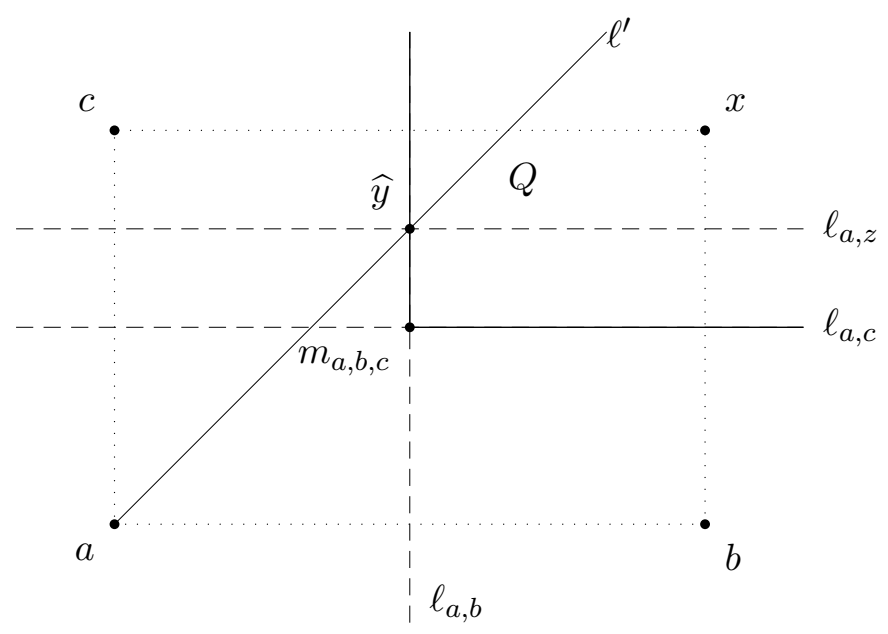

Figure 2.4: Illustrating the proof of Lemma 2.3.11, Step 2

Step 2 Let $a \in \mathcal{B}$ and let $b, c \in \mathcal{B} \backslash\{a\}, b \neq c$, such that $m_{a, b, c} \in A^{\circ} \cap \partial A_{a}$. Since condition (ii) in Lemma 2.3.9 does not hold, there is an $x \in \mathcal{B}$ such that $\{a, b, c, x\}$ is rectangular. Without loss of generality assume that $\ell(a, b)$ and $\ell(c, x)$, as well as $\ell(a, c)$ and $\ell(b, x)$ are parallel. (See Fig. 2.4 for an illustration of the proof of this step.) Denote $Q=H\left(\ell_{a, b}, x\right) \cap H\left(\ell_{a, c}, x\right)$. Then: $A_{a}=Q^{\circ} \cap A$.

In order to prove this, we first observe the following. Let $y$ be any point of $\bar{A}_{a}$ and let $\ell$ be the line through $a$ and $y$. Then $y^{\prime} \in A_{a}$ for any point $y^{\prime} \in A$ on $\ell$ on the other side of $y$ than $a$. Therefore, to prove our claim, it is sufficient to prove that $y \in \partial A_{a}$ for every $y \in \partial Q \cap A$. Suppose this were not true, without loss of generality suppose there is some point in $\ell_{a, b} \cap Q \cap A$ which is not in $\partial A_{a}$. Then, since $m_{a, b, c} \in \partial A_{a}$ and since $A_{a}$ is convex there is a $\widehat{y} \in \ell_{a, b} \cap Q \cap A$ such that $\left[m_{a, b, c}, \widehat{y}\right] \subseteq \partial A_{a}$ and such that $\left(\ell_{a, b} \cap Q\right) \backslash\left[m_{a, b, c}, \widehat{y}\right] \neq \emptyset$ but $\left(\ell_{a, b} \cap Q\right) \backslash\left[m_{a, b, c}, \widehat{y}\right] \cap \bar{A}_{a}=\emptyset$. Then there must be a $z \in \mathcal{B}$ such that $\ell_{a, z}$ intersects $\ell_{a, b}$ at the point $\widehat{y}$. Since, clearly, $\widehat{y} \in \partial A_{a} \cap A^{\circ}$ and condition (ii) in Lemma 2.3.9 does not hold, there must be a $v \in \mathcal{B}$ such that the set $\{a, z, b, v\}$ is rectangular. This implies in particular that $\ell_{a, z}$ and $\ell_{a, b}$ are perpendicular. But then $y \notin A_{a}$ for all $y$ in $Q^{\circ} \cap A$ on the straight line $\ell^{\prime}$ through $a$ and $\widehat{y}$. This is a contradiction with the observation at the beginning of the proof, since $\widehat{y} \in \partial A_{a}$. This 
completes the proof of the claim in Step 2.

Step 3 Let $a \in \mathcal{B}$. Then the set $\bar{A}_{a}$ is the intersection of $A$ with an orthant $Q_{a}$ with $p\left(Q_{a}\right) \in A^{\circ}$.

To prove this, just note that by Lemma 2.3.10 there are $b, c$ as in Step 2. The claim of Step 3 now follows from Step 2.

We can now complete the proof of the only-if direction. By Step 3, for each $a \in \mathcal{B}$ the set $\bar{A}_{a}$ is the intersection of $A$ with an orthant $Q_{a}$ with $p\left(Q_{a}\right) \in A^{\circ}$. Moreover, $A=\bigcup_{a \in \mathcal{B}} \bar{A}_{a}=\bigcup_{a \in \mathcal{B}}\left(Q_{a} \cap A\right)$. Hence by Step 1, $|\mathcal{B}|=4$, in particular $\mathcal{B}=\{a, b, c, x\}$ with $a, b, c, x$ as in Step 2. Thus, $\mathcal{B}$ is rectangular.

For the if-direction, let $\mathcal{B}$ be rectangular with $\mathcal{B}=\{v, w, y, z\}$. Clearly, among any three distinct points in $\{v, w, y, z\}$ there is a point $a$ such that $m_{a, b, c} \in \partial A_{a} \cap A^{\circ}$, where $b$ and $c$ are the other two points, but together with the fourth point they form the rectangular set $\mathcal{B}$. Hence, condition (ii) does not hold.

We can now formulate the main result of this section. Call $\varphi$ dictatorial if there is a dictator, i.e., an agent $d \in N$ such that $\varphi(p) \in \mathfrak{b}(p(d))$ for all $p \in A^{N}$.

Theorem 2.3.1. If $|\mathcal{B}| \geq 3$ and $\mathcal{B}$ is not rectangular then $\varphi$ is dictatorial.

Proof. By Lemmas 2.3.8, 2.3.9, and 2.3.11 the set of decisive coalitions is an ultrafilter. Hence, by Lemma 2.2.2, there is a $d \in N$ such that $d$ is decisive. So $\varphi$ is dictatorial with dictator $d$.

We conclude this section with two remarks. The first remark is about regular polytopes.

Remark 2.3.1. Call a polytope $A$ in $\mathbb{R}^{2}$ regular if its extreme points - the elements of the set $C$ - are the vertices of a regular polygon (equilateral triangle, square, etc.). For such a regular polytope Theorem 2.3.1 implies that $\varphi$ is dictatorial if and only if $|C| \neq 4$. Thus, on regular polytopes $A$, a strategy-proof and Pareto optimal rule is dictatorial unless $A$ is a square including its inside. 
The second remark concerns a relation between the results of Gibbard (1973) and Satterthwaite (1975) and Theorem 2.3.1.

Remark 2.3.2. Let $C=\{a, b, c\}$ such that $a, b$, and $c$ are the vertices of an acute triangle. By Theorem 2.3.1 any strategy-proof and Pareto optimal rule on $A$ must be a dictatorship. An alternative proof of this fact could work as follows. Let $\varphi$ be such a rule and first prove that $\varphi(p) \in C$ for every $p \in A^{N}$. Then, effectively, $\varphi$ is a strategy-proof and Pareto optimal social choice function on the set $C$ of alternatives, with full domain of preferences, as can be seen in Fig. 2.5(a), where the perpendicular bisectors of the edges are drawn. Hence, $\varphi$ must be a dictatorship according to the GibbardSatterthwaite theorem. If the triangle is not acute then only four strict preferences are generated (Fig. 2.5(b)) and thus the Gibbard-Satterthwaite theorem does not apply. Indeed, in this case, if the right or obtuse angle is at $c$, then $a$ and $b$ are the only single-best points, and for instance majority voting between $a$ and $b$ is a strategy-proof and Pareto optimal rule.
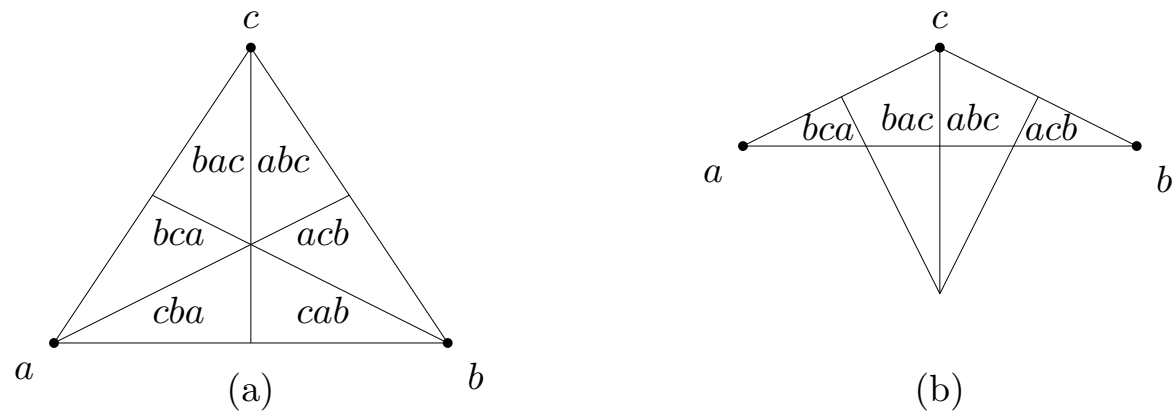

(b)

Figure 2.5: Remark 2.3.2

\subsection{Nondictatorship}

In this section we study the two cases excluded in Theorem 2.3.1, namely the case $|\mathcal{B}|=2$ and the case where $\mathcal{B}$ is rectangular. The main result in the first case - two single-best alternatives - is Theorem 2.4.1, which characterizes all Pareto optimal and strategy-proof rules for this case. In the second case - where there are exactly four single-best alternatives, 
forming the vertices of a rectangle - we content ourselves by giving an example of a nondictatorial Pareto optimal and strategy-proof rule.

\subsection{1 $|\mathcal{B}|=2$}

According to Theorem 2.3.1 dictatorship is implied by Pareto optimality and strategy-proofness under two conditions on the set $\mathcal{B}$ of single-best alternatives in $C$. In this section we consider the case where the first one of these conditions does not hold. More precisely, we assume that $|\mathcal{B}|=2$, and write $\mathcal{B}=\{a, b\}$. The following lemma reveals a consequence of this assumption. It says that the center of the circle through $a, b$, and any other point $c \in C$ cannot be an interior point of $A$.

Lemma 2.4.1. Let $c \in A \backslash\{a, b\}$. Then $m_{a, b, c} \notin A^{\circ}$.

Proof. First observe that for any $B \subseteq C$ with $|B| \geq 2$ we have that $A_{B}$, if not empty, consists of a line segment (which may happen if $|B|=2$ ) or a single point. This implies that any open subset of $A^{\circ}$ has a nonempty intersection with $A_{a}$ or $A_{b}$. Now suppose $m_{a, b, c} \in A^{\circ}$. Then the set $\left(V_{c a b}^{\circ} \cup V_{c b a}^{\circ}\right) \cap A^{\circ}$, where $V_{c a b}=\left\{x \in A:(c, a),(a, b) \in R_{x}\right\}$ and $V_{c b a}=\left\{x \in A:(c, b),(b, a) \in R_{x}\right\}$, is an open subset of $A^{\circ}$. However, $A_{a} \cap\left(V_{c a b}^{\circ} \cup V_{c b a}^{\circ}\right) \cap A^{\circ}=\emptyset$ and $A_{b} \cap\left(V_{c a b}^{\circ} \cup V_{c b a}\right)^{\circ} \cap A^{\circ}=\emptyset$. This contradicts that any open subset of $A^{\circ}$ has a non-empty intersection with $A_{a}$ or $A_{b}$, hence $m_{a, b, c} \notin A^{\circ}$.

For the remainder of this section let again $\varphi$ be a Pareto optimal and strategy-proof rule. We first show that in almost all cases we have $\varphi(p) \in$ $\{a, b\}$.

Lemma 2.4.2. Let $p \in A^{N}, p \neq m_{a, b, c}^{N}$ for all $c \in C \backslash\{a, b\}$. Then $\varphi(p) \in$ $\{a, b\}$.

Proof. To the contrary suppose $\varphi(p) \notin\{a, b\}$. We write $m$ for $m_{a, b, \varphi(p)}$ if $a, b$, and $\varphi(p)$ are not collinear; otherwise, $\varphi(p) \in] a, b[$ and we take $m=\varphi(p)$. (Below it will follow that $\varphi(p) \in \partial A$, so that in view of Lemma 2.3.1 the latter case will actually not occur.) By Lemma 2.4.1, $m \notin A^{\circ}$, which implies that $\|x-\varphi(p)\| \leq\|x-a\|$ or $\|x-\varphi(p)\| \leq\|x-b\|$ for all 
$x \in A$ (since otherwise $m$ would be an interior point of the triangle with vertices $a, b, x$ for some $x \in A$, hence an interior point of $A$ ). In particular, we have for every $i \in N$ with $p(i) \notin\{a, b, m\}$ that $\|p(i)-\varphi(p)\|<\|p(i)-a\|$ or $\|p(i)-\varphi(p)\|<\|p(i)-b\|$. If $\|p(i)-\varphi(p)\|<\|p(i)-a\|$ for some $i \in N$, then consider the profile $p^{\prime}$ with $p^{\prime}(i)=b$ and $p^{\prime}(j)=p(j)$ for all $j \neq i$. If $\varphi\left(p^{\prime}\right)=b$ then agent $i$ would manipulate from $p^{\prime}$ to $p$ since $\varphi(p) \neq b$, and if $\varphi\left(p^{\prime}\right)=a$ then agent $i$ would manipulate from $p$ to $p^{\prime}$. Hence, strategyproofness implies $\varphi\left(p^{\prime}\right) \notin\{a, b\}$. If $\|p(i)-\varphi(p)\|<\|p(i)-b\|$ for some $i \in N$ then we consider the profile $p^{\prime \prime}$ with $p^{\prime \prime}(i)=a$ and $p^{\prime \prime}(j)=p(j)$ for all $j \neq i$ and similarly derive that $\varphi\left(p^{\prime \prime}\right) \notin\{a, b\}$. Therefore we may assume that $p(N) \subseteq\{a, b, m\}$. Since $p(N) \subseteq\{a, b\}$ would contradict Lemma 2.3.6 we may further assume that $p$ is of the form $p=\left(a^{S}, b^{T}, m^{V}\right)$ for some coalitions $S, T$, and $V=N \backslash(S \cup T) \neq \emptyset$. By Pareto optimality we have that either $S$ and $T$ are non-empty or $V=N$.

Suppose that $\varphi(p) \in A^{\circ}$. Then there are points of $A$ on the straight line through $\varphi(p)$ perpendicular to $\ell(a, b)$ that Pareto dominate $\varphi(p)$ given the profile $p$, contradicting Pareto optimality of $\varphi$. Hence, $\varphi(p) \in \partial A$, so that $\varphi(p) \in C$ by Lemma 2.3.1. Thus, by the condition on $p$ in the lemma, $V \neq N$, so that $S, T, V \neq \emptyset$.

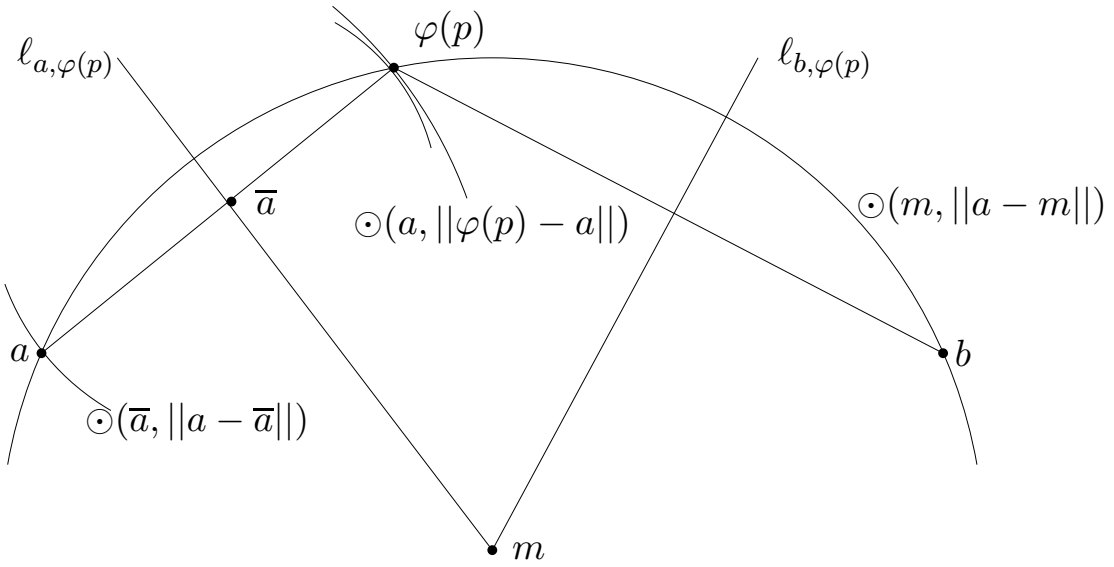

Figure 2.6: Illustrating the proof of Lemma 2.4.2

Next, let $\bar{a}=m_{a, \varphi(p)}$ and consider profile $p^{a}=\left(\bar{a}^{S}, b^{T}, m^{V}\right)$. We prove that 
$\varphi\left(p^{a}\right)=a$. (See Fig. 2.6 for an illustration of this part of the argument.) By comparing $p$ and $p^{a}$, strategy-proofness implies that $\left\|\varphi\left(p^{a}\right)-\bar{a}\right\| \geq$ $\|\varphi(p)-\bar{a}\|=\|a-\bar{a}\|$ and $\|\varphi(p)-a\| \geq\left\|\varphi\left(p^{a}\right)-a\right\|$. Consider the profile $q=\left(\bar{a}^{S}, b^{T \cup V}\right)$. Note that $b \in A_{a}$; also, since by Lemma 2.4.1, $m_{a, b, c} \notin A^{\circ}$ and $m_{a, b, c} \in \ell_{a, b}$ for all $c \in A \backslash\{a, b\}$, it follows that $\bar{a} \in A_{b}$. Now Lemma 2.3.6 implies $\varphi(q) \in\{a, b\}$, hence strategy-proofness yields $\|m-a\|=$ $\|m-b\| \leq\left\|m-\varphi\left(p^{a}\right)\right\|$. This means that $\varphi\left(p^{a}\right)$ is not inside the circle with center $m$ through the points $a, b$ and $\varphi(p)$, but then $\varphi\left(p^{a}\right)$ must be on this circle since there are no points of $A$ outside this circle (since otherwise we could find a point in $\mathcal{B}$ different from $a$ and $b$, a contradiction). Hence, $\|m-a\|=\left\|m-\varphi\left(p^{a}\right)\right\|$. Since $\|\varphi(p)-a\| \geq\left\|\varphi\left(p^{a}\right)-a\right\|,\left\|\varphi\left(p^{a}\right)-\bar{a}\right\| \geq$ $\|a-\bar{a}\|$ and $\|m-a\|=\left\|m-\varphi\left(p^{a}\right)\right\|$ it follows that either $\varphi\left(p^{a}\right)=\varphi(p)$ or $\varphi\left(p^{a}\right)$ is on the circle with center $m$ through $a$ and $\varphi(p)$ on the arc between $a$ and $b$ not containing $\varphi(p)$. The latter case, however, implies $\varphi\left(p^{a}\right)=a$ since there cannot be points of $C$ on this arc other than $a$ or $b$. Hence, $\varphi\left(p^{a}\right) \in\{a, \varphi(p)\}$. But $\varphi(p)$ is Pareto dominated by $a$ at the profile $p^{a}$. So, $\varphi\left(p^{a}\right)=a$.

Now let $\bar{b}=m_{b, \varphi(p)}$ and consider the profile $p^{a b}=\left(\bar{a}^{S}, \bar{b}^{T}, m^{V}\right)$. Note that $\bar{b} \in \mathcal{H}^{\circ}\left(\ell_{a, b}, b\right)$. Similarly as above we have $\bar{b} \in A_{a}$. Therefore $\varphi\left(p^{a b}\right)=a$ by strategy-proofness.

By an analogous argument but now starting with the profile $p^{b}=$ $\left(a^{S}, \bar{b}^{T}, m^{V}\right)$, we obtain $p^{a b}=b$, a contradiction which completes the proof.

In order to characterize all strategy-proof and Pareto optimal rules while avoiding technicalities due to preference indifferences, we make the following additional assumptions. The first assumption takes care of the cases not covered by Lemma 2.4.2. More precisely, suppose $m_{a, b, c} \in A$ for some $c \in C \backslash\{a, b\}$. Then by Lemma 2.4.1 we must have $m_{a, b, c} \in\{\underline{m}, \bar{m}\}$, where $\{\underline{m}, \bar{m}\}=\partial A \cap \ell_{a, b}$.

Assumption 2.4.1. $\varphi(p) \in\{a, b\}$ for $p=\underline{m}^{N}$ and $p=\bar{m}^{N}$.

The second assumption, which is a version of the familiar non-bossiness condition, guarantees that indifferent agents cannot change the assigned alternative. 
Assumption 2.4.2. For all $i \in N$ and $p, q \in A^{N}$ such that $p(j)=q(j)$ for all $j \in N \backslash\{i\}$, if $\|p(i)-a\|=\|p(i)-b\|$ and $\|q(i)-a\|=\|q(i)-b\|$, then $\varphi(p)=\varphi(q)$.

With these additional assumptions, it is straightforward to verify that (a strategy-proof and Pareto optimal rule) $\varphi$ depends only on the individual preferences between $a$ and $b$ and not on the exact location of the dips. We will now develop a precise description of $\varphi$.

Let $\mathcal{W}_{a}^{\varphi}$ be the set of pairs $(S, U) \in N \times N$ with $S \cap U=\emptyset$ and such that $\varphi(p)=a$ for all profiles $p$ with $a$ single-best for all agents in $S$ and both $a$ and $b$ best for all agents in $U$. Let $\mathcal{W}_{b}^{\varphi}$ be defined analogously. Since $\varphi$ depends only on the individual preferences between $a$ and $b$ we have that the pair $\left(\mathcal{W}_{a}^{\varphi}, \mathcal{W}_{b}^{\varphi}\right)$ is both proper and strong, i.e., either $(S, U) \in \mathcal{W}_{a}^{\varphi}$ or $(T, U) \in \mathcal{W}_{b}^{\varphi}$ for all pairwise disjoint sets $S, U$ and $T$ with $S \cup T \cup U=N$. Pareto-optimality of $\varphi$ implies that the pair $\left(\mathcal{W}_{a}^{\varphi}, \mathcal{W}_{b}^{\varphi}\right)$ is Pareto optimal, i.e., $(S, U) \in \mathcal{W}_{a}^{\varphi}$ if $S \neq \emptyset$ and $S \cup U=N$, and $(T, U) \in \mathcal{W}_{b}^{\varphi}$ if $T \neq \emptyset$ and $T \cup U=N$. Furthermore, strategy-proofness of $\varphi$ implies that the pair $\left(\mathcal{W}_{a}^{\varphi}, \mathcal{W}_{b}^{\varphi}\right)$ is monotone, i.e., $\left(S^{\prime}, U^{\prime}\right) \in \mathcal{W}_{a}^{\varphi}$ whenever $(S, U) \in \mathcal{W}_{a}^{\varphi}$ and $S \subseteq S^{\prime}$ and $S \cup U \subseteq S^{\prime} \cup U^{\prime}$ and $\left(T^{\prime}, U^{\prime}\right) \in \mathcal{W}_{b}^{\varphi}$ whenever $(T, U) \in \mathcal{W}_{b}^{\varphi}$ and $T \subseteq T^{\prime}$ and $T \cup U \subseteq T^{\prime} \cup U^{\prime}$. It is straightforward to check that any proper and strong, Pareto optimal and monotone pair $\left(\mathcal{W}_{a}, \mathcal{W}_{b}\right)$ defines a Pareto optimal and strategy-proof rule $\varphi$, satisfying Assumptions 2.4.1 and 2.4.2, with $\left(\mathcal{W}_{a}^{\varphi}, \mathcal{W}_{b}^{\varphi}\right)=\left(\mathcal{W}_{a}, \mathcal{W}_{b}\right)$. We summarize these findings in the following theorem.

Theorem 2.4.1. Let $\mathcal{B}=\{a, b\}$ with $a \neq b$. Then

(i) If $\varphi$ is strategy-proof, Pareto optimal, and satisfies Assumptions 2.4.1 and 2.4.2, then $\left(\mathcal{W}_{a}^{\varphi}, \mathcal{W}_{b}^{\varphi}\right)$ is proper and strong, Pareto optimal and monotone.

(ii) If $\left(\mathcal{W}_{a}, \mathcal{W}_{b}\right)$ is proper and strong, Pareto optimal and monotone, then there is a strategy-proof, and Pareto optimal rule $\varphi$, satisfying Assumptions 2.4.1 and 2.4.2, such that $\mathcal{W}_{a}=\mathcal{W}_{a}^{\varphi}$ and $\mathcal{W}_{b}=\mathcal{W}_{b}^{\varphi}$. 


\subsection{2 $\mathcal{B}$ is rectangular}

In this section we consider the case where $\mathcal{B}$ is rectangular. Thus, we assume that $\mathcal{B}=\{a, b, c, d\}$ and that $[a, b],[b, c],[c, d]$, and $[d, a]$ are the edges of a rectangle. Rather than attempting to describe all strategy-proof and Pareto efficient rules for this case - which becomes a very technical exercise mainly due to many tie-breaking decisions that have to be made we content ourselves with giving a typical example of a rule.

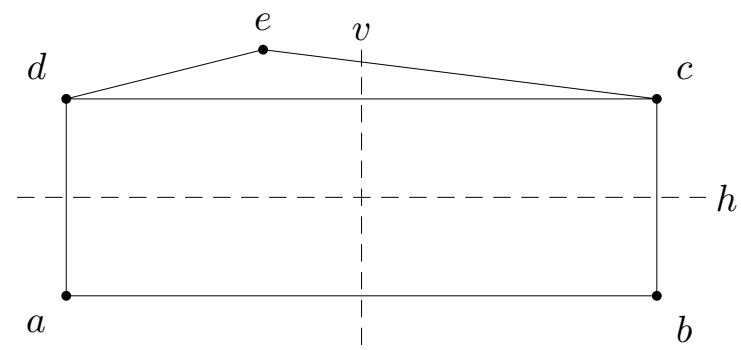

Figure 2.7: Illustrating Example 2.4.1

Example 2.4.1. (Cf. Fig 2.7.) Let $h$ be the common perpendicular bisector of $[a, d]$ and $[b, c]$ and let $v$ be the common perpendicular bisector of $[a, b]$ and $[c, d]$. For every profile $p$ define $\varphi(p)$ as follows. If $|\{i \in N: p(i) \in H(h, a)\}| \geq|\{i \in N: p(i) \in H(h, d)\}|$ then $\varphi(p) \in\{c, d\}$ and if $|\{i \in N: p(i) \in H(h, a)\}|<|\{i \in N: p(i) \in H(h, d)\}|$ then $\varphi(p) \in\{a, b\}$. If $|\{i \in N: p(i) \in H(v, a)\}| \geq|\{i \in N: p(i) \in H(v, b)\}|$ then $\varphi(p) \in\{b, c\}$ and if $|\{i \in N: p(i) \in H(v, a)\}|<|\{i \in N: p(i) \in H(v, b)\}|$ then $\varphi(p) \in\{a, d\}$. In words, we let majority voting decide between $\{a, b\}$ and $\{c, d\}$ on the one hand, and between $\{a, d\}$ and $\{b, c\}$ on the other. It can be proved (left to the reader) that this rule is strategy-proof and Pareto optimal.

\subsection{Concluding remarks}

The preceding sections naturally lead to the question if the obtained results can be extended to other regions, such as general compact convex sets. 
Since some of the proofs seem to rely heavily on the finiteness of the set of extreme points, this question does not appear to have a straightforward answer. In Chapter 3 we show that the dictatorship result extends to the disc (circle and its inside) in $\mathbb{R}^{2}$. This result may not come as a surprise in view of the fact that the disc can be seen as the 'limit' of polytopes for which Theorem 2.3.1 implies dictatorship, but nevertheless we need a separate proof to show this.

Another potential extension concerns the domain of preferences. One might expect, however, that allowing for a richer class of single-dipped preferences, other than only Euclidean, is bound to result in dictatorship. ${ }^{7}$

\footnotetext{
${ }^{7}$ This is likely but does not follow directly from our results. In principle, a rule could be dictatorial if all agents report Euclidean preferences but nondictatorial for some other profiles.
} 



\section{Chapter 3}

\section{Disc}

\subsection{Introduction and model}

This chapter contributes to the following question: Where to locate a public bad (noxious facility) like for instance a garbage dumping ground or nuclear plant within a given region? ${ }^{1}$ Specifically, we assume that a finite number of agents, equipped with single-dipped Euclidean preferences, reaches a decision by voting. The dips (worst locations) of these agents are private knowledge - e.g., residence, children's school, etc. - and thus we require the (voting) rule to be strategy-proof in order to elicit the true information about these dips. We also require the rule to be Pareto optimal, and assume the region to be a disc, i.e., a circle and its interior, in the Euclidean plane. This choice is quite specific. In Chapter 2 we study strategy-proof and Pareto optimal rules for the case where $A$ is a polytope in $\mathbb{R}^{2}$, i.e., the convex hull of finitely many points. We find that such rules can be based on majority voting and more generally voting by committees (simple games) if the region is either long-shaped or it is (basically) a rectangle and its inside. In all other cases such rules are dictatorial, including all regular polytopes except for the square. We conjecture that similar results will hold on general compact convex sets, being the 'limits' of polytopes, and in the present note

\footnotetext{
${ }^{1}$ This chapter is based on Öztürk, Peters, and Storcken (2013).
} 
indeed show dictatorship on the disc. We need, however, a separate proof and are not able to extend the result for polytopes by some limit argument - indeed, even a dictatorial rule in this model is not continuous, a small change in the dictator's dip may cause the assigned point to swap to the other side. The general case of compact convex regions in $\mathbb{R}^{2}$ is therefore still open.

Formally, we consider the following collective decision problem. The set of alternatives is the $\operatorname{disc} A=\left\{x \in \mathbb{R}^{2}:\|x\| \leq 1\right\}$, where $\|\cdot\|$ denotes Euclidean distance. The set of agents is $N=\{1, \ldots, n\}$ with $n \geq 2$. Each agent has a single-dipped Euclidean preference on $A$, characterized by a dip $a \in A$ such that $x \in A$ is weakly preferred to $y \in A$ if and only if $\|x-a\| \geq$ $\|y-a\|$. A profile is a vector $p \in A^{N}$, where $p(i)$ is the dip of agent $i$, for each $i \in N$. A rule $\varphi$ assigns to each $p \in A^{N}$ a collective decision $\varphi(p) \in A$. A rule $\varphi$ is strategy-proof if for all $i \in N$ and $p, q \in A^{N}$ with $p(j)=q(j)$ for all $j \in N \backslash\{i\}$ we have $\|\varphi(p)-p(i)\| \geq\|\varphi(q)-p(i)\|$. It is not difficult to verify that strategy-proofness is equivalent to the following property, which we will use interchangeably. A rule $\varphi$ is intermediate strategy-proof if for every coalition $S \subseteq N$ and all profiles $p, q \in A^{N}$ such that $p(j)=q(j)$ for all $j \in N \backslash S$ and there is an alternative $a$ with $p(i)=a$ for all $i \in S$, we have $\|\varphi(p)-a\| \geq\|\varphi(q)-a\|$. A rule $\varphi$ is Pareto optimal if for every $p \in A^{N}$, every $a \in A$ and every $i \in N$, if $\|a-p(i)\|>\|\varphi(p)-p(i)\|$ then there is a $j \in N$ such that $\|a-p(j)\|<\|\varphi(p)-p(j)\|$.

Other existing work on strategy-proofness under single-dipped preferences (for the public good/bad case) is confined to one-dimensional regions, specifically Peremans and Storcken (1999), Barberà et al (2012), and Manjunath (2009). In our proof below we use decisive coalitions and ultrafilters, cf. Hansson (1976). There is also a literature on locating noxious facilities if monetary transfers are possible, see e.g. Kunreuther and Kleindorfer (1986) for a relatively early reference.

\subsection{Dictatorship}

In this section we prove our main result. 
Theorem 3.2.1. Let $\varphi: A^{N} \rightarrow A$ be a rule. Then $\varphi$ is strategy-proof and Pareto optimal if and only if $\varphi$ is dictatorial, i.e., there is an agent $d$ such that $\|\varphi(p)-p(d)\| \geq\|x-p(d)\|$ for every $p \in A^{N}$ and every $x \in A$.

Observe that for every $x \in A \backslash\{0\}$ (where $0=(0,0) \in \mathbb{R}^{2}$ ) there is a unique point on the boundary $\partial A$ of $A$ that has maximal distance from $x$; we denote this point by $\widehat{x}$. In particular, if $x \in \partial A$ then $\widehat{x}=-x$. If $x=0$ then all points of $\partial A$ are at maximal distance (namely, 1) from $x$. Hence, if $\varphi$ is dictatorial with dictator $d$ then $\varphi(p)=\widehat{p(d)}$ whenever $p(d) \neq 0$, and $\varphi(p) \in \partial A$ if $p(d)=0$.

For $a, b \in \partial A$ we denote the closed arc between $a$ and $b$ by $[a \frown b]$. (If $b=-a$ then $[a \frown b]$ is defined to be $\partial A$, i.e., the full circle.) Also, $[a \frown b[=[a \frown b] \backslash\{b\}$, ]$a \frown b]=[a \frown b] \backslash\{a\}$, and $] a \frown b[=[a \frown b] \backslash\{a, b\}$.

For $x, y \in A$ and $S \subseteq N$ we denote by $p=\left(x^{S}, y^{N \backslash S}\right)$ the profile with $p(i)=x$ for all $i \in S$ and $p(i)=y$ for all $i \in N \backslash S$. The first lemma says that if $\varphi$ is Pareto optimal then it assigns a boundary point to each such two-dip profile.

Lemma 3.2.1. Let $\varphi: A^{N} \rightarrow A$ be Pareto optimal, and let $x, y \in A$ and $S \subseteq N$. Then $\varphi\left(x^{S}, y^{N \backslash S}\right) \in \partial A$.

Proof. Let $a=\varphi\left(x^{S}, y^{N \backslash S}\right)$. If $a \notin \partial A$ then let $\ell$ be the straight line through $a$ and perpendicular to the straight line $\ell^{\prime}$ through $x$ and $y$ if $x \neq y$; if $x=y$ then let $\ell$ be the straight line through $a$ and $x$. Then at least one of the two points in $\ell \cap \partial A$ Pareto dominates $a$ at the profile $\left(x^{S}, y^{N \backslash S}\right)$, a contradiction.

Until further notice, $\varphi: A^{N} \rightarrow A$ is strategy-proof and Pareto optimal.

Lemma 3.2.2. Let $a \in \partial A$ and $S \subseteq N$. Then $\varphi\left(a^{S}, \widehat{a}^{N \backslash S}\right) \in\{a, \widehat{a}\}$.

Proof. In the proof only two-dip profiles are considered: therefore, by Lemma 3.2.1, their outcomes under $\varphi$ are in $\partial A$. Contrary to what is to prove, assume that $\varphi\left(a^{S}, \widehat{a}^{N \backslash S}\right)=b$ where $b \notin\{a, \widehat{a}\}$. Without loss of generality we may assume that $\|a-b\| \leq\|\widehat{a}-b\|$. For $x, y \in \partial A$ let $y_{x}$ be the point on $\partial A$ such that $\left\{y, y_{x}\right\}=\{z \in \partial A:\|x-z\|=\|x-y\|\}$. 


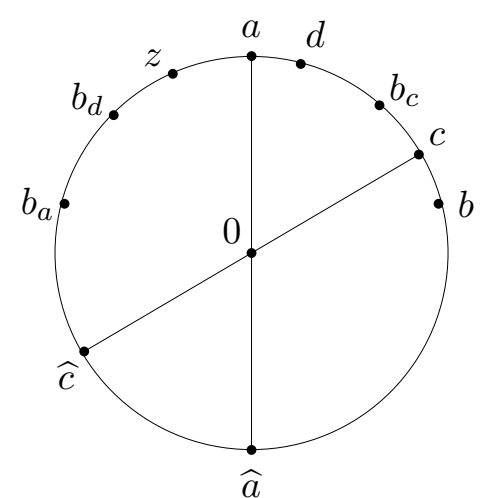

(a)

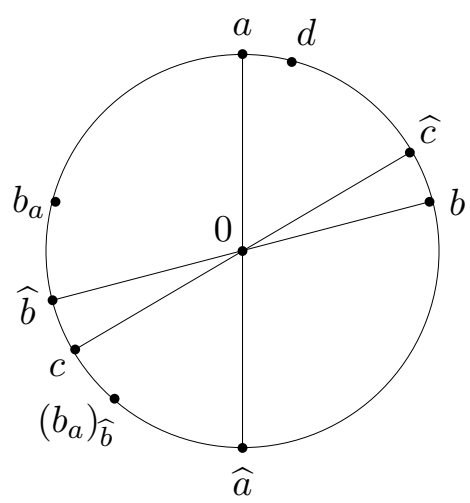

(b)

Figure 3.1: Illustrating the proof of Lemma 3.2.2; $z=\varphi\left(c^{S}, \widehat{a}^{N \backslash S}\right)$

First we prove that $\varphi\left(b^{S}, \widehat{a}^{N \backslash S}\right)=b_{a}$. (See Fig. 3.1(a).) Consider $c \in[a \frown b[$. Since $\|a-b\| \leq\|\widehat{a}-b\|$, strategy-proofness implies $\varphi\left(c^{S}, \widehat{a}^{N \backslash S}\right) \in\{b\} \cup\left[b_{c} \frown\right.$ $\left.b_{a}\right]$. Pareto optimality yields that $\varphi\left(c^{S}, \widehat{a}^{N \backslash S}\right) \in[\widehat{c} \frown a]$. Clearly $b \notin[\widehat{c} \frown a]$. So, $\varphi\left(c^{S}, \widehat{a}^{N \backslash S}\right) \in\left[b_{c} \frown b_{a}\right]$. Note that we just showed that $\varphi\left(x^{S}, \widehat{a}^{N \backslash S}\right) \in$ $\left[b_{x} \frown b_{a}\right]$ for all $x \in\left[a \frown b\left[\right.\right.$. Suppose $\varphi\left(c^{S}, \widehat{a}^{N \backslash S}\right) \neq b_{a}$. Then we can choose $d \in\left[a \frown c\left[\right.\right.$ such that $\left.\left.\left[b_{d} \frown b_{a}\right] \subseteq\right] \varphi\left(c^{S}, \widehat{a}^{N \backslash S}\right), b_{a}\right]$. Since $\varphi\left(d^{S}, \widehat{a}^{N \backslash S}\right) \in\left[b_{d} \frown b_{a}\right]$ this implies that $\left\|\varphi\left(d^{S}, \widehat{a}^{N \backslash S}\right)-c\right\|>\left\|\varphi\left(c^{S}, \widehat{a}^{N \backslash S}\right)-c\right\|$, which obviously contradicts strategy-proofness. So, $\varphi\left(c^{S}, \widehat{a}^{N \backslash S}\right)=b_{a}$. Since the choice of $c$ was arbitrary this proves that $\varphi\left(x^{S}, \widehat{a}^{N \backslash S}\right)=b_{a}$ for all $x \in[a \frown b[$. Strategyproofness now implies that $\varphi\left(b^{S}, \widehat{a}^{N \backslash S}\right)=b_{a}$.

Next we prove (see Fig. 3.1(b)) that $\varphi\left(b^{S}, \widehat{b}^{N \backslash S}\right) \in\left\{b_{a},\left(b_{a}\right)_{\widehat{b}}\right\}$. Consider $c \in] \widehat{b} \frown \widehat{a}\left[\right.$. Since $\varphi\left(b^{S}, \widehat{a}^{N \backslash S}\right)=b_{a}$, strategy-proofness implies that $\varphi\left(b^{S}, c^{N \backslash S}\right) \in\left\{b_{a}\right\} \cup\left[\left(b_{a}\right)_{c} \frown b\right]$. Since Pareto optimality implies $\varphi\left(b^{S}, c^{N \backslash S}\right) \subseteq[\widehat{b} \frown \widehat{c}]$ it follows that $\varphi\left(b^{S}, c^{N \backslash S}\right)=b_{a}$. So we proved that $\varphi\left(b^{S}, x^{N \backslash S}\right)=b_{a}$ for all $\left.x \in\right] \widehat{b} \frown \widehat{a}[$. Strategy-proofness now implies that $\varphi\left(b^{S}, \widehat{b}^{N \backslash S}\right) \in\left\{b_{a},\left(b_{a}\right)_{\widehat{b}}\right\}$.

Finally we prove $\varphi\left(b^{S}, \widehat{b}^{N \backslash S}\right)=b$, which is a contradiction since $b \notin$ $\left\{b_{a},\left(b_{a}\right)_{\hat{b}}\right\}$, and thus completes the proof. Since $\varphi\left(a^{S}, \widehat{a}^{N \backslash S}\right)=b$, strategyproofness implies $\varphi\left(a^{S}, \widehat{b}^{N \backslash S}\right)=b$. For $d \in[a \frown b[$ strategy-proofness implies $\varphi\left(d^{S}, \widehat{b}^{N \backslash S}\right) \in\{b\} \cup\left[b_{d} \frown b_{a}\right]$. Pareto optimality now implies that $\varphi\left(d^{S}, \widehat{b}^{N \backslash S}\right)=b$. By the arbitrary choice of $d$ we have $\varphi\left(x^{S}, \widehat{b}^{N \backslash S}\right)=b$ for 
all $x \in\left[a \frown b\left[\right.\right.$. So strategy-proofness implies $\varphi\left(b^{S}, \widehat{b}^{N \backslash S}\right)=b$.

The next lemma shows that at two-dip profiles as in Lemma 3.2.2 always the same coalition gets its best point.

Lemma 3.2.3. Let $S \subseteq N$. Then either $\varphi\left(x^{S}, \widehat{x}^{N \backslash S}\right)=\widehat{x}$ for all $x \in \partial A$ or $\varphi\left(x^{S}, \widehat{x}^{N \backslash S}\right)=x$ for all $x \in \partial A$.

Proof. Let $a, b \in \partial A$. In view of Lemma 3.2.2 we may assume without loss of generality that $\varphi\left(a^{S}, \widehat{a}^{N \backslash S}\right)=\widehat{a}$. It is sufficient to prove that $\varphi\left(b^{S}, \widehat{b}^{N \backslash S}\right)=\widehat{b}$. We distinguish two cases.

Case 1: $\{a, \widehat{a}\} \cap\{b, \widehat{b}\}=\emptyset$. To the contrary, suppose $\varphi\left(b^{S}, \widehat{b}^{N \backslash S}\right) \neq \widehat{b}$. Then Lemma 3.2.2 yields $\varphi\left(b^{S}, \widehat{b}^{N \backslash S}\right)=b$. Strategy-proofness and $\varphi\left(a^{S}, \widehat{a}^{N \backslash S}\right)=$ $\widehat{a}$ imply $\varphi\left(a^{S}, \widehat{b}^{N \backslash S}\right)=\widehat{a}$. But strategy-proofness and $\varphi\left(b^{S}, \widehat{b}^{N \backslash S}\right)=b$ imply $\varphi\left(a^{S}, \widehat{b}^{N \backslash S}\right)=b$. This yields the contradiction $b=\widehat{a}$.

Case 2: $b=\widehat{a}$. So, $\{a, \widehat{a}\}=\{b, \widehat{b}\}$. Take $c \in \partial A \backslash\{a, \widehat{a}, b, \widehat{b}\}$. Applying the previous case yields first $\varphi\left(c^{S}, \widehat{c}^{N \backslash S}\right)=\widehat{c}$ and by this result that $\varphi\left(b^{S}, \widehat{b}^{N \backslash S}\right)=\widehat{b}$.

Call a coalition $S \subseteq N$ decisive if for every $a \in A$ and every profile $p \in A^{N}$ with $p(i)=a$ for all $i \in N$ we have $\varphi(p)=\widehat{a}$ if $a \neq 0$ and $\varphi(p) \in \partial A$ if $a=0$. The next two lemmas deal with decisive coalitions.

Lemma 3.2.4. Let $S \subseteq N$. Then $S$ is decisive or $N \backslash S$ is decisive.

Proof. In view of Lemma 3.2.3, we may assume without loss of generality that $\varphi\left(a^{S}, \widehat{a}^{N \backslash S}\right)=\widehat{a}$ for all $a \in \partial A$. We prove that $S$ is decisive. Take $b \in A$ and $p \in A^{N}$ such that $p(i)=b$ for all $i \in S$. It is sufficient to prove that $\varphi(p)$ is at maximal distance from $b$. We distinguish two cases.

Case $1: \quad b \neq 0$. Let $c=-\widehat{b}$. By assumption $\varphi\left(c^{S}, \widehat{b}^{N \backslash S}\right)=\widehat{b}$. But then strategy-proofness implies $\left\|b-\varphi\left(b^{S}, \widehat{b}^{N \backslash S}\right)\right\| \geq\|b-\widehat{b}\|$. Hence, $\varphi\left(b^{S}, \widehat{b}^{N \backslash S}\right)=\widehat{b}$. Strategy-proofness now implies $\varphi(p)=\widehat{b}$.

Case 2: $b=0$. By the previous case it follows that $\varphi(q) \in \partial A$ for all profiles $q$ such that $q(j)=p(j)$ for all $j \in N \backslash S$ and $q(i)=x$ for all $i \in S$, for some $x \in A \backslash\{0\}$. Now strategy-proofness implies that $\varphi(p) \in \partial A$.

Lemma 3.2.5. Let $S, T \subseteq N$ be both decisive. Then $S \cap T$ is decisive. 
Proof. To the contrary suppose that $S \cap T$ is not decisive. Then, by Lemma 3.2.4, $N \backslash(S \cap T)$ is decisive. Let $X=S \backslash T, Y=S \cap T$, and $Z=N \backslash S$. So $X \cup Y=S, Y \cup Z=N \backslash(S \backslash T) \supseteq T$ and $X \cup Z=N \backslash(S \cap T)$ are decisive. Further, let $p=\left(a^{X}, b^{Y}, c^{Z}\right)$ with $a, b, c \in \partial A$ (hence agents in $X$ have their dips at $a$, agents in $Y$ at $b$, and agents in $Z$ at $c$ ), and such that $a, b$, and $c$ are the vertices of an equilateral triangle. (See Fig. 3.2(a).) Since $X \cup Y$ is

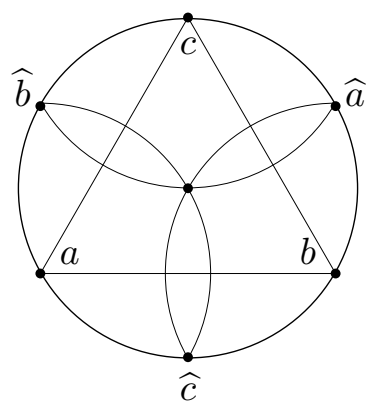

(a)

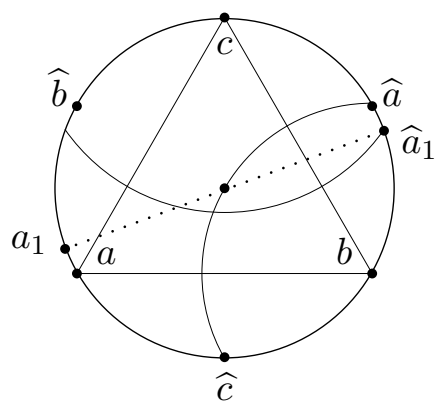

(b)

Figure 3.2: Illustrating the proof of Lemma 3.2.5

decisive, hence $\varphi\left(b^{X \cup Y}, c^{Z}\right)=\widehat{b}$, it follows by strategy-proofness that

$$
\|a-\varphi(p)\| \geq\|a-\widehat{b}\| .
$$

Similarly, since $X \cup Z$ and $Y \cup Z$ are decisive we have $\varphi\left(a^{X \cup Z}, b^{Y}\right)=\widehat{a}$ and $\varphi\left(a^{X}, c^{Y \cup Z}\right)=\widehat{c}$ so that strategy-proofness implies that

$$
\|c-\varphi(p)\| \geq\|c-\widehat{a}\| \text { and }\|b-\varphi(p)\| \geq\|b-\widehat{c}\| .
$$

Therefore, $\varphi(p) \in\{0, \widehat{a}, \widehat{b}, \widehat{c}\}$, but 0 is Pareto dominated by for instance $\widehat{a}$. Without loss of generality, assume that $\varphi(p)=\widehat{a}$. Take $\left.a_{1} \in\right] a \frown \widehat{b}[$ close to $a$. By applying the above argument with $a_{1}$ instead of $a$, we find for the profile $p^{\prime}=\left(a_{1}^{X}, b^{Y}, c^{Z}\right)$ in particular the inequalities $\left\|c-\varphi\left(p^{\prime}\right)\right\| \geq\left\|c-\widehat{a}_{1}\right\|$ and $\left\|b-\varphi\left(p^{\prime}\right)\right\| \geq\|b-\widehat{c}\|$. For points $x \in A$ satisfying these inequalities we have (see Fig. 3.2(b)) $\left\|a_{1}-x\right\| \leq\left\|a_{1}-\widehat{c}\right\|$, hence in particular $\left\|a_{1}-\varphi\left(p^{\prime}\right)\right\| \leq$ $\left\|a_{1}-\widehat{c}\right\|$. Since $\left\|a_{1}-\widehat{c}\right\|<\left\|a_{1}-\widehat{a}\right\|$ by the choice of $a_{1}$, and since $\varphi(p)=\widehat{a}$, coalition $X$ can manipulate from $p^{\prime}$ to $p$, and thus we have a violation of strategy-proofness. 
By Pareto optimality and Lemmas 3.2.4 and 3.2.5 the collection $\mathcal{D}$ of decisive coalitions satisfies (i) $\emptyset \notin \mathcal{D}$, (ii) if $S, T \in \mathcal{D}$, then $S \cap T \in \mathcal{D}$, and (iii) $S \in \mathcal{D}$ or $N \backslash S \in \mathcal{D}$. Thus, $\mathcal{D}$ is an ultrafilter and it is well-known and straightforward to prove that there is a unique $d \in N$ with $\{d\} \in \mathcal{D}$. Clearly then, $\varphi$ is dictatorial with dictator $d$. This proves the only-if direction of Theorem 3.2.1. Since, trivially, a dictatorial rule is strategy-proof and Pareto optimal, the proof of Theorem 3.2.1 is complete. 



\section{Chapter 4}

\section{Trees}

\subsection{Introduction}

In this chapter, the problem of locating a public bad in a tree network is examined. A public bad is a necessary public facility with side effects that make agents dislike being close to it. A garbage dump site is an example of a public bad. Society, a finite number of agents, will collectively decide where to locate this public bad along a tree network, i.e. graph. The nature of public bad restricts an agent's preference to be single-dipped. That is there exists a worst location, the dip, and preference increases by moving away from this dip.

A solution is a social choice function which assigns an outcome on the graph to any possible single-dipped preference profile, i.e. combination of individual preferences. Solutions are assumed to satisfy the conditions: Pareto optimality (for each outcome there is no alternative which is at least as good as for all agents and strictly better for some agents), strategy-proofness (a deviating agent cannot gain from this change), non-corruptibility (if a deviating agent is indifferent, then the outcome cannot change) and anonymity (names of the agents do not matter).

The most important concept in this chapter is extreme leaves. The diameter of a graph is the length of longest distance available in this graph. An 
extreme leaf is an end point of a path of length equal to the diameter. Note that, if the length of a path is equal to the diameter of the graph, then the endpoints of this path will be two extreme leaves. We show that for any single-dipped profile, the outcome of a Pareto optimal, strategy-proof, non-corrupt and anonymous solution is an extreme leaf.

Next we show that Pareto optimal, strategy-proof, non-corrupt and anonymous solutions exist. But, instead of characterizing these solutions, an example, which picks the outcome from the set of weak Condorcet winners, is given. The main result of this chapter is the equivalence of these four conditions and pairwise monotonicity (the outcome will not change if it improves when it is compared to each extreme leaf) when preferences are single-dipped over a tree network.

Related literature To the author's knowledge, single-dipped preferences on a tree is an untouched topic in domain restrictions. On the other hand, single-peaked preferences, which can be seen as the counterpart to singledipped preferences, are explicitly studied. Demange (1982) shows that the set of single-peaked preferences on a tree implies the existence of a majority winner. Later, Danilov (1994) characterizes strategy-proof social choice rules in this framework. More recently, Vohra (1999), Klaus (2001a) and Schummer and Vohra (2002) study social choice rules with desired conditions when preferences are single-peaked on a tree.

Organization of this chapter Section 4.2 starts with notations, the basic models and some preliminary results. Section 4.3 gives results over pairwise monotonicity.

\subsection{Basic Notations, The Model and Preliminar- ies}

In this section we fix the notations, state the model and preliminaries that will be used throughout the chapter. 


\subsubsection{Notations}

The set $N=\{1,2, \ldots, n\}$ denotes the finite set of agents. Agents collectively locate a public bad on a connected graph $G$, i.e. an ordered pair $(V, E)$ where $V$ is the finite set of vertices in the Euclidean plane and $E$ is the finite set of edges. An edge between vertices $v$ an $w$ is the closed line segment between $v$ and $w$ in the Euclidean plane, and edges do not intersect with each other except at their endpoints. Further, it is assumed that $G$ is a tree, i.e. it does not contain cycles. A vertex which is on only one edge is called a leaf, and the set of all leaves will be denoted by $L$. The set of possible locations for the public bad is defined by the union of all edges $A=\cup\{e: e \in E\}$. Let $x$ and $y$ be two points in $A$. A path between $x$ and $y$, denoted by $[x, y]$, is the intersection of all connected subsets of $A$ that contain $x$ and $y$. The notations $] x, y],[x, y[$ and $] x, y[$ will denote paths excluding indicated endpoints, i.e. $[x, y] \backslash\{x\},[x, y] \backslash\{y\}$ and $[x, y] \backslash\{x, y\}$ respectively. Further, we assume that $A$ is endowed with a distance function. Denoting the distance between $x$ and $y$ in $A$ by $x y$, we assume that for $z$ on path $[x, y]$

$$
x y=x z+z y .
$$

The diameter $d_{G}$ of graph $G$ is the length of a longest path on the graph: $d_{G}=\max _{x, y \in A} x y$. Obviously for $x, y \in A$ with $x y=d_{G}$, we have $x, y \in L$. We call leaves that are endpoints of a longest path extreme leaves, and denote the set of extreme leaves by $\mathcal{L} \subseteq L$. For every location $x$ in $A$, the number $\gamma_{x}$ denotes the number of connected components of $A \backslash\{x\}$. Although it is defined only for vertices in graph theory, we will abuse the notation and call this number grade of $x$. Let each of these components be represented by $\mathcal{H}(x, y)$, where $y$ is an arbitrary point of this component. Note that for two disjoint component $\mathcal{H}(x, y)$ and $\mathcal{H}(x, z)$ the point $x$ is on path $[y, z]$. For each component $\mathcal{H}(x, y)$ we may find locations, say $x^{\varepsilon}$, possibly close to $x$ such that for all locations $z$ on $\left.] x, x^{\varepsilon}\right]$ the grade $\gamma_{z}=2$. So, $x^{\varepsilon}$ is on every path $[x, v]$ for all $v \in \mathcal{H}(x, y) \backslash\left[x, x^{\varepsilon}\right]$. For any $x, y \in A$ we denote the midpoint of $[x, y]$ by $m(x, y)$, i.e. $x m(x, y)=m(x, y) y$.

Each agent has a (single-dipped) preference $R$ which is defined as follows; 
there is some point $x$ in $A$ such that any point $y$ in $A$ is weakly preferred to a point $z$ in $A$ if $x y \geq x z$. Point $x$ is said to be the dip of preference $R$. Clearly preference $R$ is completely determined by its dip. Therefore we will denote preferences by their dips. Let $p \in A^{N}$ be a profile, i.e. a combination of individual preferences $p=(p(1), p(2), \ldots, p(n))$. Then $p(i)$ denotes both agents $i$ 's dip and preference. A coalition $S$ is a subset of $N$.

\subsubsection{The model}

A solution is a function $\varphi: A^{N} \rightarrow A$, which assigns an outcome $\varphi(p) \in A$ to any profile $p \in A^{N}$.

Pareto Optimality A solution $\varphi$ is called Pareto optimal, if for all profiles $p \in A^{N}$ there exists no $a \in A$ with $p(i) a \geq p(i) \varphi(p)$ for all $i \in N$ and $p(j) a>p(j) \varphi(p)$ for some $j \in N$.

Anonymity A solution $\varphi$ is called anonymous, if $\varphi$ is symmetric in its arguments.

Strategy-proofness A solution $\varphi$ is called strategy-proof, if for all agents $i$ in $N$ and all profiles $p$ and $q$ in $A^{N}$, with $q(j)=p(j)$ for all $j \in N \backslash\{i\}$, we have $p(i) \varphi(p) \geq p(i) \varphi(q)$.

Now we will define intermediate strategy-proofness. The reader can verify that strategy-proofness and intermediate strategy-proofness coincide by using the proof in Peters et al. (1992), since the proof depends on the transitivity of the preferences. In this chapter, we will mainly use intermediate strategy-proofness without mentioning it explicitly.

Intermediate Strategy-proofness A solution $\varphi$ is called intermediate strategy-proof, if for all coalitions $S \subseteq N$ and all profiles $p$ and $q$ in $A^{N}$, such that $p(j)=q(j)$ for all $j \in N \backslash S$ and further such that there is an alternative $a$ with $p(i)=a$ for all $i \in S$, we have $a \varphi(p) \geq a \varphi(q)$.

Non-corruptness A solution $\varphi$ is called non-corrupt if for all profiles $p$ and $q$ in $A^{N}$ and all agents $i$ in $N$ if $p(j)=q(j)$ for all $j \in N \backslash\{i\}$ and if $p(i) \varphi(p)=p(i) \varphi(q)$ and $q(i) \varphi(p)=q(i) \varphi(q)$, then $\varphi(p)=\varphi(q)$. 
In this chapter, unless mentioned otherwise, a solution $\varphi: A^{N} \rightarrow A$ will be strategy-proof, Pareto optimal, anonymous and non-corrupt.

\subsubsection{Preliminaries}

First we will show that for any tree shaped graph $G$, there exists a unique point $o_{G}$ which is equidistant to any extreme leaf of this graph.

Proposition 4.2.1. There exists a unique midpoint $o_{G}$, such that $l_{G}=$ $\frac{1}{2} d_{G}$ for all $l \in \mathcal{L}$.

Proof. Let $a, b, x$ and $y$ be extreme leaves such that $a b=x y=d_{G}$. It is sufficient to prove that the midpoint $m(a, b)$ of $[a, b]$ is equal to the midpoint $m(x, y)$ of $[x, y]$, which implies $o_{G}=m(a, b)=m(x, y)$. To the contrary suppose $m(x, y) \neq m(a, b)$. It is sufficient to deduce a contradiction. As $\mathcal{H}(m(a, b), a) \cap \mathcal{H}(m(a, b), b)=\emptyset$, we may assume that $m(x, y) \notin$ $\mathcal{H}(m(a, b), b)$. So, $m(a, b) \in] m(x, y), b]$. As $\mathcal{H}(m(x, y), x) \cap \mathcal{H}(m(x, y), y)=$ $\emptyset$, we may assume that $\mathcal{H}(m(x, y), m(a, b)) \cap \mathcal{H}(m(x, y), x)=\emptyset$. So, $m(x, y) \in] m(a, b), x]$. Figure 4.1 illustrates the proof.

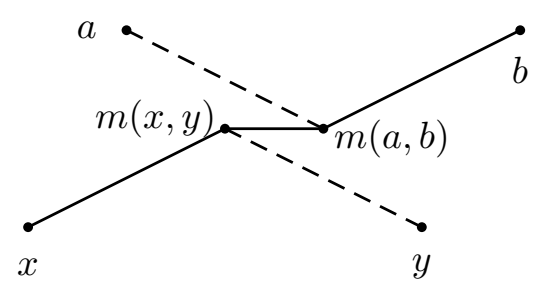

Figure 4.1: Illustrating the proof of Proposition 4.2.1

Hence $x b=x m(x, y)+m(x, y) m(a, b)+m(a, b) b=\frac{1}{2} d_{G}+m(x, y) m(a, b)+$ $\frac{1}{2} d_{G}>d_{G}$. This contradiction ends the proof.

Now $A \backslash\left\{o_{G}\right\}$ yields connected components, say $A_{1}$ up to say $A_{\gamma_{o_{G}}}$. Clearly $\gamma_{o_{G}} \geq 2$. Define $\mathcal{L}_{i}=\mathcal{L} \cap A_{i}$ for $i \in\left\{1, \ldots, \gamma_{o_{G}}\right\}$. As $o_{G}$ is on the path between any two extreme leaves $l$ and $l^{\prime}$ which are in different components $\mathcal{L}_{j}$ and $\mathcal{L}_{k}$ it follows that $\left[l, o_{G}\right] \cap\left[l^{\prime}, o_{G}\right]=\left\{o_{G}\right\}$ and that $l l^{\prime}=d_{G}$. 
Lemma 4.2.1. Let $v, w \in A^{N}$ with $v(i) \in[w(i), \varphi(v)]$ for all agents $i$ in $N$. Then $\varphi(v)=\varphi(w)$.

Proof. Let there be precisely one agent, say $i$, such that his preference at $v$ differs from that at $w$. Strategy-proofness implies that $v(i) \varphi(v) \geq v(i) \varphi(w)$ and $w(i) \varphi(w) \geq w(i) \varphi(v)$. So,

$$
\begin{aligned}
& w(i) \varphi(w) \leq w(i) v(i)+v(i) \varphi(w) \quad \text { (triangle inequality) } \\
& \leq w(i) v(i)+v(i) \varphi(v) \quad \text { (strategy-proofness) } \\
& =\quad w(i) \varphi(v) \quad(v(i) \in[w(i), \varphi(v)]) \\
& \leq \quad w(i) \varphi(w) \quad \text { (strategy-proofness). }
\end{aligned}
$$

So, the foregoing three inequalities are actually equalities. Hence, $v(i) \varphi(v)=v(i) \varphi(w)$ and $w(i) \varphi(v)=w(i) \varphi(w)$. As $\varphi$ is non-corrupt this yields $\varphi(v)=\varphi(w)$. Now, the proof is complete by repeating this argument for all agents in $N$.

Now we will prove that for any profile $p \in A^{N}$, the outcome of a strategyproof, Pareto optimal, anonymous and non-corrupt solution must be one of the extreme leaves.

Proposition 4.2.2. Let $p \in A^{N}$. Then $\varphi(p) \in \mathcal{L}$.

Proof. To the contrary assume $\varphi(p) \notin \mathcal{L}$. Consider a path $[a, b]$ such that $a b=d_{G}$. First we prove that $m_{a}=m(a, \varphi(p))$ and $m_{b}=m(b, \varphi(p))$ are different points on $[a, b]$. Let $[a, c]=[a, b] \cap[a, \varphi(p)]$. In order to prove $m_{a} \neq m_{b}$ and $m_{a}$ is on $[a, b]$ it is sufficient to show that $m_{a} \in[a, c[$. Because $A$ has a tree structure we have $[b, \varphi(p)]=[\varphi(p), c] \cup[c, b]$. So, $m_{a} \notin[a, c[$ yields $c \varphi(p)>c a$. Hence, as $\varphi(p) b=\varphi(p) c+c b$, we have $\varphi(p) b>c a+c b=d_{G}$. By this contradiction to our assumptions we may conclude $m_{a} \in[a, c[$.

Next we show that $\left.o_{G} \in\right] m_{a}, m_{b}\left[\right.$. Note that $m_{b} c+c \varphi(p)=m_{b} b$, and $a m_{a}+m_{a} c>c \varphi(p)$. Adding these two yields $a m_{a}+m_{a} c+m_{b} c+c \varphi(p)>$ $m_{b} b+c \varphi(p)$. So, $a m_{a}+m_{a} c+m_{b} c>m_{b} b$, which yields that $a m_{b}>m_{b} b$. So, $o_{G} \in\left[a, m_{b}\right]$. Similarly it follows that $o_{G} \in\left[b, m_{a}\right]$. Hence, $o_{G} \in$ $\left[m_{a}, m_{b}\right]$. Since $\varphi(p) \notin \mathcal{L}$, we get $\left.o_{G} \in\right] m_{a}, m_{b}[$. Figure 4.2 illustrates the situation. 


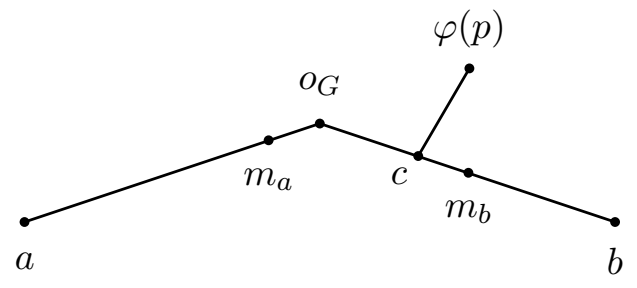

Figure 4.2: Illustrating the proof of Proposition 4.2.2

Take $m_{a}^{\varepsilon}$ in $\mathcal{H}\left(m_{a}, a\right)$ close to $m_{a}$ such that $m_{a} a>m_{a}^{\varepsilon} m_{a}$ and no dip $p(i)$ is in $\left[m_{a}^{\varepsilon}, m_{a}\right.$ [ and all points $x$ on $\left[m_{a}^{\varepsilon}, m_{a}\right]$ have grade number $\gamma_{x}=2$. So, for all points $y$ in $\mathcal{H}\left(m_{a}, a\right) \backslash\left[m_{a}^{\varepsilon}, m_{a}\right]$ point $m_{a}^{\varepsilon}$ is on path $\left[y, m_{a}\right]$. Consider an agent $j$ in $N$ such that $p(j)$ is in $\mathcal{H}\left(m_{a}, a\right)$.

Take profile $q$ which differs from $p$ only for agent $j$. For this agent $q(j)=m_{a}^{\varepsilon}$. We argue that $\varphi(p)=\varphi(q)$. First we show that $\varphi(q)$ is not in $\mathcal{H}\left(m_{a}, a\right) \cup\left\{m_{a}\right\}$. Strategy-proofness requires $q(j) \varphi(q) \geq q(j) \varphi(p)$. As by construction $q(j) \varphi(p)=m_{a}^{\varepsilon} \varphi(p)>m_{a} a$ and $m_{a} a>m_{a}^{\varepsilon} m_{a}$, it follows that $\varphi(q) \notin\left[m_{a}^{\varepsilon}, m_{a}\right]$. Also as $m_{a}^{\varepsilon}$ is on any path $\left[y, m_{a}\right]$ for $y \in \mathcal{H}\left(m_{a}, a\right) \backslash\left[m_{a}^{\varepsilon}, m_{a}\right]$ we have $\varphi(q) \notin \mathcal{H}\left(m_{a}, a\right)$. Because else, $\varphi(q) b=$ $\varphi(q) q(j)+q(j) b \geq q(j) \varphi(p)+q(j) b=q(j) \varphi(p)+m_{a}^{\varepsilon} m_{a}+m_{a} b>m_{a} a+$ $m_{a}^{\varepsilon} m_{a}+m_{a} b>a b$, which contradicts that $a b=d_{G}$. So, $q(j) \in[p(j), \varphi(q)]$, because $m_{a}^{\varepsilon} \in\left[p(j), m_{a}\right] \subseteq[p(j), \varphi(q)]$. But now Lemma 4.2.1, with $p$ in the role of $w$ and $q$ in that of $v$, yields $\varphi(p)=\varphi(q)$.

By possibly repeating the previous argument we may assume that $p(j)=$ $m_{a}^{\varepsilon}$ for all agents $j$ with $p(j) \in \mathcal{H}\left(m_{a}, a\right)$. Further, we may assume that $p(j)=m_{b}^{\varepsilon}$ for all agents $j$ with $p(j) \in \mathcal{H}\left(m_{b}, b\right)$, where $m_{b}^{\varepsilon}$ is a point in $\mathcal{H}\left(m_{b}, b\right)$ close to $m_{b}$ similarly defined to $m_{a}^{\varepsilon}$. Let $r^{a} \in A^{N}$ with $r^{a}(j)=m_{a}$ if $p(j)=m_{a}^{\varepsilon}$ and $r^{a}(j)=p(j)$ otherwise, let $r^{b} \in A^{N}$ with $r^{b}(j)=m_{b}$ if $p(j)=m_{b}^{\varepsilon}$ and $r^{b}(j)=p(j)$ otherwise and let $r \in A^{N}$ with $r(j)=m_{a}$ if $p(j)=m_{a}^{\varepsilon}, r(j)=m_{b}$ if $p(j)=m_{b}^{\varepsilon}$ and $r(j)=p(j)$ otherwise.

Consider profiles $p$ and $r^{a}$. First, we have $\varphi\left(r^{a}\right) \neq \varphi(p)$, as at $r^{a}$ point $\varphi(p)$ is Pareto dominated by $a$. Note that $r^{a}(i)=m_{a}$ for all $i$ with $r^{a}(i) \in$ $\mathcal{H}\left(m_{a}, a\right)$, so these agents are indifferent between $a$ and $\varphi(p)$, and rest of the agents have dips in $A \backslash \mathcal{H}\left(m_{a}, a\right)$, so they strictly prefer $a$ to $\varphi(p)$. 
Next, note that strategy-proofness and $m_{a}^{\varepsilon}$ being close to $m_{a}$ implies that $\varphi\left(r^{a}\right) \neq m_{a}$. Now $\varphi\left(r^{a}\right) \in \mathcal{H}\left(m_{a}, a\right)$ because otherwise we would have that $r^{a}(i) \in\left[p(i), \varphi\left(r^{a}\right)\right]$ for $i$ with $p(i) \in \mathcal{H}\left(m_{a}, a\right)$, which would yield contradiction $\varphi\left(r^{a}\right)=\varphi(p)$ by Lemma 4.2.1 with $r^{a}$ in the role of $v$ and $p$ in that of $w$. Further, strategy-proofness yields $\varphi\left(r^{a}\right) m_{a} \geq \varphi(p) m_{a}=a m_{a}$. This implies that $\varphi\left(r^{a}\right)$ is an extreme leaf in $\mathcal{H}\left(m_{a}, a\right)$. Similarly, we have that $\varphi\left(r^{b}\right)$ is an extreme leaf and in $\mathcal{H}\left(m_{b}, b\right)$. Considering profiles $r^{a}$ and $r$ it follows by Lemma 4.2.1 that $\varphi\left(r^{a}\right)=\varphi(r)$. Similarly we have $\varphi\left(r^{b}\right)=\varphi(r)$. These two contradict each other as obviously $\varphi(r)$ cannot be in both $\mathcal{H}\left(m_{a}, a\right)$ and $\mathcal{H}\left(m_{b}, b\right)$.

Now we will give an example of Pareto optimal, strategy-proof, non-corrupt and anonymous solution. Before defining the solution, we will define weak Condorcet winner in a profile and show that for any profile there is always an extreme leaf, which is weak Condorcet winner. Location $a$ is a weak Condorcet winner at profile $p$ if it is not beaten by any other alternative at $p$. Further we define that alternative $x$ beats alternative $y$ at profile $p$ if $\eta(p, x \succ y)>\eta(p, y \succ x)$. Denote the set of weak Condorcet winner at $p$ by $\mathcal{W}(p)$. Now we show that for a profile $p$ the set $\mathcal{W}(p)$ is non-empty and it contains an extreme leaf.

Lemma 4.2.2. Let $p$ be a profile. Then $\mathcal{W}(p) \neq \emptyset$ and $\mathcal{W}(p) \cap \mathcal{L} \neq \emptyset$.

Proof. First we will proof that there exists $l \in \mathcal{L}$ such that $l$ is not beaten by any point $x \in A$. For any $A_{i}$ with $i \in\left\{1, \ldots, \gamma_{o_{G}}\right\}$ and $p \in A^{N}$, let $h_{i}(p)$ be the number of dips in the component $A_{i}$, i.e. $h_{i}(p)=\#\{j \in N \mid p(j) \in$ $\left.A_{i}\right\}$. Note that for any $l, l^{\prime} \in \mathcal{L}$ with $l l^{\prime}=d_{G}, l$ beats $l^{\prime}$ if and only if $h_{j}(p)>h_{i}(p)$. W.l.o.g., let $h_{1}(p) \leq h_{i}(p)$ for all $i \in\left\{1, \ldots, \gamma_{o_{G}}\right\}$. We will show that the relation "beat" is transitive in $\mathcal{L}_{1}$, which will imply that there exists an extreme leaf in $\mathcal{L}_{1}$ that is not beaten any other extreme leaf in $\mathcal{L}_{1}$. Let $l, l^{\prime}, l^{\prime \prime} \in \mathcal{L}_{1}$ with $l$ beats $l^{\prime}$ and $l^{\prime}$ beats $l^{\prime \prime}$. We will show that $l$ beats $l^{\prime \prime}$, which shows that beat relation is transitive. Note that $l$ beats $l^{\prime}$ means that $\eta\left(p, l \succ l^{\prime}\right)>\eta\left(p, l^{\prime} \succ l\right)$, and $l^{\prime}$ beats $l^{\prime \prime}$ means that $\eta\left(p, l^{\prime} \succ l^{\prime \prime}\right)>\eta\left(p, l^{\prime \prime} \succ l^{\prime}\right)$. We will distinguish the following cases based on the positions of $m\left(l^{\prime}, l^{\prime \prime}\right)$ and $m\left(l, l^{\prime}\right)$.

Case $1 m\left(l^{\prime}, l^{\prime \prime}\right) \in \mathcal{H}\left(m\left(l, l^{\prime}\right), l^{\prime}\right)$. Then $l^{\prime \prime} \in \mathcal{H}\left(m\left(l, l^{\prime}\right), l^{\prime}\right)$, otherwise 
$\left.m\left(l^{\prime}, l^{\prime \prime}\right) \in\right] m\left(l, l^{\prime}\right), l^{\prime}\left[\right.$ and $l^{\prime \prime} m\left(l, l^{\prime}\right)<l^{\prime} m\left(l, l^{\prime}\right)$. But this will contradict with $l^{\prime \prime} \in \mathcal{L}$ because $o_{G} l^{\prime \prime} \leq o_{G} m\left(l, l^{\prime}\right)+m\left(l, l^{\prime}\right) l^{\prime \prime}<o_{G} m\left(l, l^{\prime}\right)+m\left(l, l^{\prime}\right) l^{\prime}=\frac{1}{2} d_{G}$. Further, $m\left(l^{\prime}, l^{\prime \prime}\right)=m\left(l, l^{\prime}\right)$. Which implies that $\eta\left(p, l \succ l^{\prime \prime}\right)=\eta\left(p, l \succ l^{\prime}\right)$ and $\eta\left(p, l^{\prime \prime} \succ l\right)=\eta\left(p, l^{\prime} \succ l\right)$. So $l$ beats $l^{\prime \prime}$.

Case $2 m\left(l^{\prime}, l^{\prime \prime}\right) \in \mathcal{H}\left(m\left(l, l^{\prime}\right), l\right)$. We have two subcases;

Subcase 2.1 $l^{\prime \prime} \in \mathcal{H}\left(m\left(l, l^{\prime}\right), l\right)$. Then $l^{\prime \prime} m\left(l^{\prime}, l^{\prime \prime}\right)=m\left(l^{\prime}, l^{\prime \prime}\right) m\left(l, l^{\prime}\right)+m\left(l, l^{\prime}\right) l^{\prime}$. But this will imply contradiction with $l \in \mathcal{L}$, because $\operatorname{lm}\left(l, l^{\prime}\right)=l^{\prime} m\left(l, l^{\prime}\right)$ and

$$
\begin{aligned}
l_{G} & =\operatorname{lm}\left(l, l^{\prime}\right)+m\left(l, l^{\prime}\right) o_{G} \\
& <m\left(l^{\prime}, l^{\prime \prime}\right) m\left(l, l^{\prime}\right)+\operatorname{lm}\left(l, l^{\prime}\right)+m\left(l, l^{\prime}\right) o_{G} \\
& =m\left(l^{\prime}, l^{\prime \prime}\right) m\left(l, l^{\prime}\right)+l^{\prime} m\left(l, l^{\prime}\right)+m\left(l, l^{\prime}\right) o_{G} \\
& =l^{\prime} m\left(l^{\prime}, l^{\prime \prime}\right)+m\left(l, l^{\prime}\right) o_{G} \\
& =l^{\prime \prime} m\left(l^{\prime}, l^{\prime \prime}\right)+m\left(l, l^{\prime}\right) o_{G} \\
& <m\left(l^{\prime}, l^{\prime \prime}\right) m\left(l, l^{\prime}\right)+l^{\prime \prime} m\left(l^{\prime}, l^{\prime \prime}\right)+m\left(l, l^{\prime}\right) o_{G} \\
& =l^{\prime \prime} o_{G} .
\end{aligned}
$$

Subcase 2.2 $l^{\prime \prime} \notin \mathcal{H}\left(m\left(l, l^{\prime}\right), l\right)$. Then $\left.m\left(l, l^{\prime \prime}\right) \in\right] m\left(l, l^{\prime}\right), l\left[\right.$ and $l^{\prime \prime} m\left(l, l^{\prime}\right)<$ $\operatorname{lm}\left(l, l^{\prime}\right)$. But this will contradict with $l^{\prime \prime} \in \mathcal{L}$ because $o_{G} l^{\prime \prime} \leq o_{G} m\left(l, l^{\prime}\right)+$ $m\left(l, l^{\prime}\right) l^{\prime \prime}<o_{G} m\left(l, l^{\prime}\right)+m\left(l, l^{\prime}\right) l=\frac{1}{2} d_{G}$.

Case $3 m\left(l^{\prime}, l^{\prime \prime}\right)=m\left(l, l^{\prime}\right)$. Then $\eta\left(p, l^{\prime \prime} \succ l^{\prime}\right)=\eta\left(p, l \succ l^{\prime}\right)$. We have two subcases;

Subcase $3.1 l^{\prime \prime} \in \mathcal{H}\left(m\left(l, l^{\prime}\right), l\right)$. Then $\eta\left(p, l^{\prime} \succ l^{\prime \prime}\right)=\eta\left(p, l^{\prime} \succ l\right)$, which contradicts with $l^{\prime}$ beats $l^{\prime \prime}$.

Subcase 3.2 $l^{\prime \prime} \notin \mathcal{H}\left(m\left(l, l^{\prime}\right), l\right)$. Then $\eta\left(p, l \succ l^{\prime \prime}\right)=\eta\left(p, l \succ l^{\prime}\right)>\eta\left(p, l^{\prime} \succ\right.$ $l)=\eta\left(p, l^{\prime} \succ l^{\prime \prime}\right)>\eta\left(p, l^{\prime \prime} \succ l^{\prime}\right)=\eta\left(p, l^{\prime \prime} \succ l\right)$. So $l$ beats $l^{\prime \prime}$.

Case $4 m\left(l^{\prime}, l^{\prime \prime}\right) \in \mathcal{H}\left(m\left(l, l^{\prime}\right), o_{G}\right)$. Then $m\left(l, l^{\prime \prime}\right)=m\left(l^{\prime}, l^{\prime \prime}\right)$, so $\eta\left(p, l^{\prime \prime} \succ\right.$ $l)=\eta\left(p, l^{\prime \prime} \succ l^{\prime}\right)$ and $\eta\left(p, l \succ l^{\prime \prime}\right)=\eta\left(p, l^{\prime} \succ l^{\prime \prime}\right)$. So $l$ beats $l^{\prime \prime}$. This case concludes that $l$ beats $l^{\prime \prime}$.

Let $\bar{l}$ be an extreme leaf in $\mathcal{L}_{1}$ that is not beaten by any other leaf in $\mathcal{L}_{1}$. The choice of $\mathcal{L}_{1}$ implies that $\bar{l}$ cannot be beaten by any other $l^{\prime} \in \mathcal{L}_{j}$ with 
$j \in\left\{1, \ldots, \gamma_{o_{G}}\right\} \backslash\{i\}$. Next we will show that $\bar{l}$ is not beaten by any point in $A$. Let $x \in A \backslash \mathcal{L}$, then $m(\bar{l}, x) \in] \bar{l}, o\left[\right.$ which implies $\mathcal{H}(m(\bar{l}, x), l) \subset \mathcal{H}\left(o_{G}, \bar{l}\right)$ and $\mathcal{H}\left(o_{G}, l^{\prime}\right) \subset \mathcal{H}(m(\bar{l}, x), x)$ for any $l^{\prime} \in \mathcal{L}_{j}$ with $\left.j \in\left\{1, \ldots, \gamma_{o_{G}}\right\} \backslash\{i\}\right\}$. $\mathcal{H}(m(\bar{l}, x), \bar{l}) \subset \mathcal{H}(o, \bar{l})$ implies $\eta(p, x \succ \bar{l}) \leq \eta\left(p, l^{\prime} \succ \bar{l}\right)$, and $\mathcal{H}\left(o, l^{\prime}\right) \subset$ $\mathcal{H}(m(\bar{l}, x), x)$ implies $\eta(p, \bar{l} \succ x) \leq \eta\left(p, \bar{l} \succ l^{\prime}\right)$. Since we have $\eta\left(p, l^{\prime} \succ \bar{l}\right) \leq$ $\eta\left(p, \bar{l} \succ l^{\prime}\right)$, we get $\eta(p, x \succ \bar{l}) \leq \eta(p, \bar{l} \succ x)$ which states that $x$ cannot beat $\bar{l}$. So we showed that $\bar{l} \in \mathcal{W}(p)$.

Next we will define a solution, which picks an extreme leaf from the set of weak Condorcet winners.

Example 4.2.1. Let $a_{1}, a_{2}, \ldots, a_{l}$ be a numbering of the elements in $\mathcal{L}$ such that $a_{s} a_{t} \leq a_{s} a_{u}$ and $a_{t} a_{u} \leq a_{s} a_{u}$ for all numbers $1 \leq s<t<u \leq l$. Consider rule $\varphi_{\mathcal{W}}$ defined for an arbitrary profile $p$ by $\varphi_{\mathcal{W}}(p)=a_{s}$ such that $s$ is the smallest index such that $a_{s} \in \mathcal{W}(p)$. Then $\varphi_{\mathcal{W}}$ is Pareto optimal, strategy-proof, non-corrupt and anonymous. Remains to discuss the numbering $a_{1}, a_{2}, \ldots, a_{l}$. First of all $a_{1}$ can be chosen freely among $\mathcal{L}$. Next there is a partition $\left\{X_{0}, X_{1}, \ldots, X_{k}\right\}$ of $\mathcal{L}$ such that $a_{1} x \leq a_{1} y$, whenever $x \in X_{i}, y \in X_{j}$ and $i \leq j$. Next note that $x y<x z$ for all $x, y \in X_{i}$ and all $z \in \mathcal{L} \backslash X_{i}$. So, repeating the partition on $A \mid X_{i}$ several times yields obviously such a numbering.

Now we will show the logical independence of these four conditions by giving four examples of solutions which satisfy three of the conditions while not satisfying the last one. In these examples we will use the numbering that we define for $\varphi_{\mathcal{W}}$ for tie-breaking.

Example 4.2.2. Consider the dictatorship $\varphi_{d}$ of agent $d \in N$ that picks the extreme leaf with the smallest index among extreme leaves that are in $\mathcal{W}(p)$ and at maximum distance to the dip of agent $d$. The solution $\varphi_{d}$ will be Pareto optimal, strategy-proof and non-corrupt, but not anonymous.

Example 4.2.3. Consider the constant rule; $\varphi_{c}(p)=a_{1}$ for all $p \in A^{N}$. The solution $\varphi_{c}$ will be strategy-proof, non-corrupt and anonymous, but not Pareto optimal.

Example 4.2.4. Let $\varphi_{W}^{\prime}$ be slightly modified version of $\varphi_{W}$ as follows; $\varphi_{W}^{\prime}(p)=a_{2}$ if $\varphi_{W}(p)=a_{1}, a_{1} a_{2} \neq d_{G}$ and $p(i) a_{1}=d_{G}$ for all $i \in N$. The 
solution $\varphi_{W}^{\prime}$ will be Pareto optimal, strategy-proof and anonymous, but corruptable.

Example 4.2.5. Let $A=[0,1]$. Consider the solution $\varphi_{\text {inf }}=0$ if $\mathcal{W}(p)=$ $\{0\}$ and $\varphi_{\text {inf }}=\inf (\mathcal{W}(p) \backslash\{0\})$ else. The solution $\varphi_{\text {inf }}$ will be Pareto optimal, anonymous and non-corrupt, but not strategy-proof.

\subsection{Pairwise Monotonicity}

Let $p$ be a profile and let $a$ and $b$ two extreme leaves, i.e. $a, b \in \mathcal{L}$. Let $\eta(p, a \succ b)=\#\{i \in N: p(i) \in \mathcal{H}(m(a, b), b)\}$ be the number of agents who strictly prefer $a$ to $b$ at profile $p$. Now we will define a monotonicity condition.

Pairwise Monotonicity A solution $\varphi$ is pairwise monotone if for all profiles $p$ and $q$, we have $\varphi(p)=\varphi(q)$ whenever $\eta(q, \varphi(p) \succ x) \geq \eta(p, \varphi(p) \succ x)$ and $\eta(p, x \succ \varphi(p)) \geq \eta(q, x \succ \varphi(p))$ for all $x$ in $\mathcal{L}$.

Next we show that non-corrupt, strategy-proof, anonymous and Paretooptimal rules are pairwise monotone.

Lemma 4.3.1. Let $\varphi$ be a non-corrupt, strategy-proof, Pareto optimal and anonymous rule. Then $\varphi$ is pairwise monotone.

Proof. Let $p$ and $q$ be profiles such that $\eta(q, \varphi(p) \succ x) \geq \eta(p, \varphi(p) \succ x)$ and $\eta(p, x \succ \varphi(p)) \geq \eta(q, x \succ \varphi(p))$ for all $x$ in $\mathcal{L}$. It is sufficient to prove that $\varphi(p)=\varphi(q)$. Let $\left\{X_{0}, X_{1}, \ldots, X_{k}\right\}$ be a partition of $\mathcal{L}$ such that $\varphi(p) x \leq \varphi(p) y$, whenever $x \in X_{i}, y \in X_{j}$ and $i \leq j$. Consequently, $X_{0}=\{\varphi(p)\}$. For $i \in\{1,2, \ldots, k\}$ let $m_{i}=m\left(\varphi(p), x_{i}\right)$ where $x_{i}$ is some leaf chosen in $X_{i}$. For $i \in\{1,2, \ldots, k\}$ define $\varkappa_{i}(p)=\eta\left(p, \varphi(p) \succ x_{i}\right)$ and $\xi_{i}(p)=\eta\left(p, x_{i} \succ \varphi(p)\right)$. Define $\varkappa_{i}(q)$ and $\xi_{i}(q)$ similarly. Because of our assumption on $p$ and $q$ we have that $\varkappa_{i}(p) \leq \varkappa_{i}(q)$ and $\xi_{i}(p) \geq \xi_{i}(q)$ for all $i \in\{1,2, \ldots, k\}$. Therefore, by anonymity we may assume that for any $i \in\{1,2, \ldots, k\}$ and for agents $j$ such that $q(j) \in \mathcal{H}\left(m_{i}, \varphi(p)\right)$ also $p(j) \in \mathcal{H}\left(m_{i}, \varphi(p)\right)$ and for agents $j$ such that $p(j) \in \mathcal{H}\left(m_{i}, x_{i}\right)$ also $q(j) \in$ $\mathcal{H}\left(m_{i}, x_{i}\right)$. Let $r^{0}$ up to $r^{n}$ be profiles such that $r^{t}(j)=p(j)$ if $j>t$ and $r^{t}(j)=q(j)$ if $j \leq t$. So, $r^{0}=p, r^{n}=q$ and $r^{t-1}$ and $r^{t}$ form the unilateral 
deviation in agent $t$ for all $t \in\{1, \ldots, n\}$. We prove that $\varphi\left(r^{t}\right)=\varphi(p)$ for all $t \in\{1, \ldots, n\}$. First we prove that $\varphi\left(r^{1}\right)=\varphi(p)$. Suppose to the contrary that $\varphi(p) \neq \varphi\left(r^{1}\right)$. Let $\varphi\left(r^{1}\right) \in X_{i}$ for some $i$ in $\{1,2, \ldots, k\}$. Because of strategy-proofness $\left(\varphi(p), \varphi\left(r^{1}\right)\right) \in p(1)$ and $\left(\varphi\left(r^{1}\right), \varphi(p)\right) \in q(1)$. Which means that respectively $p(1) \notin \mathcal{H}\left(m_{i}, \varphi(p)\right)$ and $q(1) \notin \mathcal{H}\left(m_{i}, x_{i}\right)$. But then by the assumption on $p$ and $r^{1}$ we have that $p(1) \notin \mathcal{H}\left(m_{i}, x_{i}\right)$ and $q(1) \notin \mathcal{H}\left(m_{i}, \varphi(p)\right)$. So, $p(1), q(1) \notin \mathcal{H}\left(m_{i}, \varphi(p)\right) \cup \mathcal{H}\left(m_{i}, x_{i}\right)$, which means that at both $p(1)$ and $q(1)$ the outcomes $\varphi(p)$ and $\varphi\left(r^{1}\right)$ are indifferent. This contradicts the rule being non-corrupt. Therefore $\varphi\left(r^{1}\right)=\varphi(p)$. Similarly it follows that $\varphi\left(r^{1}\right)=\varphi\left(r^{2}\right), \varphi\left(r^{2}\right)=\varphi\left(r^{3}\right), \ldots$ and $\varphi\left(r^{n-1}\right)=\varphi(q)$. So, $\varphi\left(r^{t}\right)=\varphi(p)$ for all $t \in\{1, \ldots, n\}$.

Remark 4.3.1. Let $p$ and $q$ be two profiles which only differ for agent j. Further, let $x$ and $y$ be two extreme leaves in $\mathcal{L}$ and $p(j), q(j) \in$ $\mathcal{H}(m(x, y), x)$. Then for all $z \in \mathcal{L} \backslash \mathcal{H}(m(x, y), x)$ and all $u \in \mathcal{L}$ we have that $\eta(p, z \succ u)=\eta(q, z \succ u)$ and $\eta(p, u \succ z)=\eta(q, u \succ z)$. So, in that case for a pairwise monotone rule $\varphi(p)=\varphi(q)$, whenever $\varphi(p)$ or $\varphi(q)$ is not in $\mathcal{H}(m(x, y), x)$.

The following result shows that pairwise monotonicity implies anonymity, strategy-proofness and non-corruptibility.

Lemma 4.3.2. Let $\varphi$ be a pairwise monotone rule. Then $\varphi$ is anonymous, strategy-proof and non-corrupt.

Proof. Obviously pairwise monotonicity implies anonymity. Therefore we only prove strategy-proofness and non-corruptibility. Let $p$ and $q$ be two profiles which only differ for agent $j$.

(Strategy-proofness) It is sufficient to prove that $(\varphi(p), \varphi(q)) \in p(j)$. To the contrary suppose $(\varphi(p), \varphi(q)) \notin p(j)$ and consequently $\varphi(p) \neq \varphi(q)$. Let $m=m(\varphi(p), \varphi(q))$. Then $p(j) \in \mathcal{H}(m, \varphi(p))$. In case $q(j) \in$ $\mathcal{H}(m, \varphi(p)) \cup \mathcal{H}(m, \varphi(q))$, then $\eta(p, \varphi(q) \succ x) \geq \eta(q, \varphi(q) \succ x)$ and $\eta(p, x \succ \varphi(q)) \leq \eta(q, x \succ \varphi(q))$ for all $x$ in $\mathcal{L}$. So, pairwise monotonicity implies the contradiction $\varphi(q)=\varphi(p)$. Therefore we may suppose that $q(j) \notin \mathcal{H}(m, \varphi(p)) \cup \mathcal{H}(m, \varphi(q))$. Let $m^{\varepsilon}$ be a point in $\mathcal{H}(m, \varphi(p))$ close to $m$. Consider the profile $r$ which differs from $p$ and $q$ only for agent $j$. For that agent $j$ the $\operatorname{dip} r(j)=m^{\varepsilon}$. Consider $p$ and $r$. By Remark 4.3 .1 with 
$p, r, \varphi(p)$ and $\varphi(q)$ in the role of respectively $p, q, x$ and $y$ it follows that $\varphi(r) \in \mathcal{H}(m, \varphi(p))$. Because, in case the latter does not hold, then this and the Remark 4.3.1 imply the contradiction $\varphi(p)=\varphi(r) \notin \mathcal{H}(m, \varphi(p))$. But as $q(j) \notin \mathcal{H}(m, \varphi(p))$ and $m^{\varepsilon}$ may be arbitrarily close to $m$ we have $\eta(r, \varphi(r) \succ x) \leq \eta(q, \varphi(r) \succ x)$ and $\eta(q, x \succ \varphi(r)) \geq \eta(r, x \succ \varphi(r))$ for all $x$ in $\mathcal{L}$. So, in that case pairwise monotonicity implies the contradiction $\varphi(q)=\varphi(r) \in \mathcal{H}(m, \varphi(p))$.

(Non-corruptibility) Let further $\varphi(p) p(j)=\varphi(q) p(j)$ and $\varphi(q) q(j)=$ $\varphi(p) q(j)$. It is sufficient to prove that $\varphi(p)=\varphi(q)$. To the contrary suppose $\varphi(p) \neq \varphi(q)$. As $\varphi(p) p(j)=\varphi(q) p(j)$ and $\varphi(q) q(j)=\varphi(p) q(j)$ it therefore follows that $p(j)$ and $q(j)$ are in $A \backslash(\mathcal{H}(m(\varphi(p), \varphi(q)), \varphi(p)) \cup$ $\mathcal{H}(m(\varphi(p), \varphi(q)), \varphi(q)))$. Let $r$ be the profile which differs from $p$ and $q$ only for agent $j$, where $r(j)=m^{\prime}$, where $m^{\prime}$ is such that $\left[m(\varphi(p), \varphi(q)), m^{\prime}\right]=$ $[m(\varphi(p), \varphi(q)), p(j)] \cap[m(\varphi(p), \varphi(q)), q(j)]$. Note that $\eta(p, x \succ y) \geq$ $\eta(r, x \succ y)$ and $\eta(p, y \succ x) \leq \eta(r, y \succ x)$ for all $x \in \mathcal{L} \backslash \mathcal{H}\left(m^{\prime}, p(j)\right)$ and for all $y \in \mathcal{L}$. So, if $\varphi(r) \in \mathcal{L} \backslash \mathcal{H}\left(m^{\prime}, p(j)\right)$, then by pairwise monotonicity we have $\varphi(r)=\varphi(p)$ and therewith $\varphi(r) \in \mathcal{L} \backslash \mathcal{H}\left(m^{\prime}, q(j)\right)$. Similarly it follows that from $\varphi(r) \in \mathcal{L} \backslash \mathcal{H}\left(m^{\prime}, q(j)\right)$ that $\varphi(r)=\varphi(q)$ and $\varphi(r) \in \mathcal{L} \backslash \mathcal{H}\left(m^{\prime}, p(j)\right)$. This yields the contradiction $\varphi(p)=\varphi(r)=\varphi(q)$.

Corollary 4.3.1. Let $\varphi$ be a Pareto optimal rule. Then $\varphi$ is non-corrupt, anonymous and strategy-proof if and only if it is pairwise monotone.

We will conclude the chapter by showing that the solution $\varphi_{\mathcal{W}}$ is also pairwise monotone.

Example 4.3.1. We will show that $\varphi_{\mathcal{W}}$ is pairwise monotone. For the latter let $p$ and $q$ be two profiles such that $a_{s} \in \mathcal{W}(p)$ and $s$ is the smallest index such that $a_{s} \in \mathcal{W}(p)$. Further, let $\eta\left(p, a_{s} \succ x\right) \geq \eta\left(q, a_{s} \succ x\right)$ and $\eta\left(p, x \succ a_{s}\right) \leq \eta\left(q, x \succ a_{s}\right)$ for all $x \in \mathcal{L}$. It is sufficient to proof that $s$ is the smallest index such that $a_{s} \in \mathcal{W}(q)$. By the assumptions $\eta\left(p, a_{s} \succ x\right) \geq$ $\eta\left(q, a_{s} \succ x\right)$ and $\eta\left(p, x \succ a_{s}\right) \leq \eta\left(q, x \succ a_{s}\right)$ for all $x \in \mathcal{L}$ and $a_{s} \in \mathcal{W}(p)$ we have that $a_{s} \in \mathcal{W}(q)$. We may assume without loss of generality that $p$ and $q$ only differ for agent $j$. To the contrary let $a_{t} \in \mathcal{W}(q) \backslash \mathcal{W}(p)$ and $t<s$. We prove that this leads to a contradiction. Then there are $a_{u}$ which beat $a_{t}$ at $p$ 
but do not so at $q$. So, $p(j) \in \mathcal{H}\left(m\left(a_{t}, a_{u}\right), a_{t}\right)$ and $q(j) \notin \mathcal{H}\left(m\left(a_{t}, a_{u}\right), a_{t}\right)$ or $p(j) \notin \mathcal{H}\left(m\left(a_{t}, a_{u}\right), a_{u}\right)$ and $q(j) \in \mathcal{H}\left(m\left(a_{t}, a_{u}\right), a_{u}\right)$. For convenience denote $a=a_{s}, b=a_{t}$ and $c=a_{u}$. We distinguish therefore two cases.

Case $p(j) \in \mathcal{H}(m(b, c), b)$ and $q(j) \notin \mathcal{H}(m(b, c), b)$

Subcase $p(j) \in \mathcal{H}(m(a, b), b)$. Then by the assumption $\eta(p, a \succ x) \geq$ $\eta(q, a \succ x)$ for all $x \in \mathcal{L}$ it follows that $q(j) \in \mathcal{H}(m(a, b), b) \backslash \mathcal{H}(m(b, c), b)$. So, $m(b, c) \in \mathcal{H}(m(a, b), b)$. As $c$ beats $b$ at $p$ all $c^{\prime}$ in $\mathcal{H}(m(b, c), c) \cap \mathcal{L}$ beat all $b^{\prime}$ in $\mathcal{H}(m(b, c), b) \cap \mathcal{L}$. Further as $b$ is not beaten by $a$ at profile $q$ we have that all $c^{\prime}$ in $\mathcal{H}(m(b, c), c) \cap \mathcal{L}$ are not beaten by all $a^{\prime}$ in $\mathcal{L} \backslash \mathcal{H}(m(a, b), b)$. So, at $p$ there are weak Condorcet winners in $\mathcal{H}(m(b, c), c) \cap \mathcal{L}$, say $c^{\prime \prime}$. But as $b c^{\prime \prime}<b a$ it follows by the assumption on the numbering of $\mathcal{L}$ that $s$ is not the minimal index such that $a_{s}$ is in $\mathcal{W}(p)$. This contradiction completes the proof of the subcase at hand.

Subcase $p(j) \notin \mathcal{H}(m(a, b), b)$. So, $p(j) \in \mathcal{H}(m(b, c), b) \backslash \mathcal{H}(m(a, b), b)$ and $m(a, b) \in \mathcal{H}(m(b, c), b)$. But then as $c$ beats $b$ at $p$ it also beats $a$ at $p$. As this contradicts the assumption that $a$ is a weak Condorcet winner at $p$ we have completed the proof of this subcase and therewith that of this case.

Case $p(j) \notin \mathcal{H}(m(b, c), b) \cup \mathcal{H}(m(b, c), c)$ and $q(j) \in \mathcal{H}(m(b, c), c)$

Subcase $m(a, b) \in \mathcal{H}(m(b, c), b) \cup\{m(b, c)\}$. As $c$ beats $b$ at $p$ it will also beat $a$ at $p$. This cannot be as $a$ is a weak Condorcet winner at $p$. Therefore we proved this subcase.

Subcase $m(a, b) \notin \mathcal{H}(m(b, c), b) \cup\{m(b, c)\}$. Then $m(b, c) \in \mathcal{H}(m(a, b), b)$ and similarly to the first subcase we have that there are $c^{\prime \prime} \in \mathcal{W}(p) \cap$ $\mathcal{H}(m(b, c), c) \cap \mathcal{L}$. This then contradicts the numbering of the indices as $b c^{\prime \prime}<b a$. All in all this completes the proof of $\varphi_{\mathcal{W}}$ being pairwise monotone. 


\section{Chapter 5}

\section{Two Neighboring Countries}

\subsection{Introduction}

Consider a shore that is along two neighboring regions, and one of the regions has a windmill on its beach part. This paper examines the problem of locating a windmill in the other region. A windmill is an example of a public bad; i.e. a facility that is needed by society, but everyone wants to be remote from it due to its negative side effects. The nature of a public bad imposes agents to have single-dipped preferences. A preference is called single-dipped if there is a worst point, the dip, and preference increases the further we move away from this point. Since we have two public bads, we will assume that only the closer one matters. The dips of the agents are private information.

Let Left and Right be the two neighboring regions, and assume that Right has already located its public bad. The shore that is shared by Left and Right is represented by the closed interval between -1 and 1 on the real line. The interval $[-1,0]$ is the shore of Left and the interval $[0,1]$ is the shore of Right. 
Solutions are assumed to satisfy Pareto optimality, anonymity, strategyproofness and a condition called the "far away condition". The first three conditions are defined in the usual way. The far away condition assumes that when every agent is indifferent between two possible outcome locations, then the location that is further away from the other region should be selected.

Organization of this chapter The chapter assumes that the location for the public bad of Right has already been decided. Section 5.2 starts with notations and the basic models.Section 5.3 characterizes Pareto optimal solutions that satisfy the far away condition, when Right locates its public bad on the boundary between the two regions. Section 5.4 characterizes Pareto-optimal, strategy-proof and anonymous solutions that satisfy the far away condition, when Right locates its public bad in the interior of its territory and only two agents are living in the Left region. Section 5.4 also includes one of the most important results of this chapter; there exists a solution that picks an inner point as an outcome.

Section 5.5 characterizes Pareto-optimal, strong strategy-proof (an extension of the strategy-proofness condition such that a deviating agent cannot get an outcome which is further away although he might be indifferent) solutions that satisfy the far away condition, when Right locates its public bad in the interior of its territory and more than two agents are living in the Left region. Section 5.6 characterizes Pareto-optimal, strategy-proof solutions that satisfy the far away condition, when Right locates its public bad as far as possible from Left. In both sections, it is shown that the range of such solutions is on the boundaries of Left.

\subsection{Basic Notations and the Model}

Let $A \subset \mathbb{R}$ be the interval $[-1,1]$, which is partitioned into two regions, $A_{L}=[-1,0]$ and $A_{R}=[0,1] . A_{L}$ is called Left region and $A_{R}$ is called Right. Let $\alpha \in A_{L}$ denote the location of the public bad in Left and $\beta \in A_{R}$ that of Right. 
Let $|x-y|$ be the Euclidean distance between $x, y \in A$.

Let $N$ be the finite set of agents. Each agent $i$ in $N$ has a worst point, the dip, indicated by $p(i)$, from where utility does not decline when moving the closest public bad away from this dip such that a pair $\left(x_{1}, y_{1}\right) \in A_{L} \times A_{R}$ is weakly preferred to pair $\left(x_{2}, y_{2}\right) \in A_{L} \times A_{R}$ if $\min \left\{\left|p(i)-x_{1}\right|,\left|p(i)-y_{1}\right|\right\} \geq$ $\min \left\{\left|p(i)-x_{2}\right|,\left|p(i)-y_{2}\right|\right\}$. Note that this preference is determined by the $\operatorname{dip} p(i)$. Therefore we identify these preferences with their dip. Further, let the asymmetric, strict, part of preference $p(i)$ be indicated by $p^{*}(i)$. We shall take a profile $p \in A_{L}^{N}$ as a collection of dips of agents in $A_{L}$.

For any $x, y \in A$, let $\mu(x, y) \in A$ be the mid point between $x$ and $y$. Hence, $\mu(x, y)=\frac{1}{2}(x+y)$.

For any given location $\beta$ of the public bad of Right, we investigate the problem of determining the public bad location at a profile $p(i) \in A_{L}$ for all $i \in N$, for the Left region. With abuse of notation, we write $x p(i) y$ instead of $(x, \beta) p(i)(y, \beta)$ for any $p(i) \in A_{L}$ and $x, y \in A_{L}$. With respect to $p(i)$, the set of best points over $A_{L}$ is denoted by $\mathfrak{b}(p(i))=\left\{x \in A_{L} \mid x p(i) y\right.$ for all $\left.y \in A_{L}\right\}$.

We distinguish the following four cases, based on the location $\beta$ and the number of agents; (i) $\beta=0$, (ii) $\beta \in] 0,1[$ with $|N|=2$, (iii) $\beta \in] 0,1[$ with $|N|>2$ and (iv) $\beta=1$.

When $\beta \in[0,1[$, preferences can be categorized in two types: Type-I and Type-II. A preference $p(i)$ is of Type-I if $p(i) \in[-1, \mu(-1, \beta)]$. Note that, $\mathfrak{b}(p(i)) \in\{\{-1\},\{0\},\{-1,0\}\}$ and the preference is strict on either side of the dip. A preference $p(i)$ is of Type-II if $p(i) \in] \mu(-1, \beta), 0]$. Let $p(i)$ be a Type-II preference, we call $\kappa(p(i))=-2 p(i)-\beta$ the kink point of $p(i)$. Note that $\mathfrak{b}(p(i))=[-1, \kappa(p(i))]$ with $|p(i)-\beta|=|p(i)-\kappa(p(i))|$, the preference is strict on the right side of the dip and strict on the left side of the dip until the kink point. See Fig. 5.1 for examples.

Let $N^{I}(p)=\{i \in N \mid p(i)$ is of Type-I $\}$ and $N^{I I}(p)=\{i \in N \mid p(i)$ is of Type-II $\}$ denote the sets of agents with Type-I and Type-II preference, respectively. If $N^{I I} \neq \emptyset$, we define the kink point of profile $p$, say $\kappa(p)$, as follows; $\kappa(p)=\min \left\{\kappa(p(i)) \mid i \in N^{I I}(p)\right\}$. 


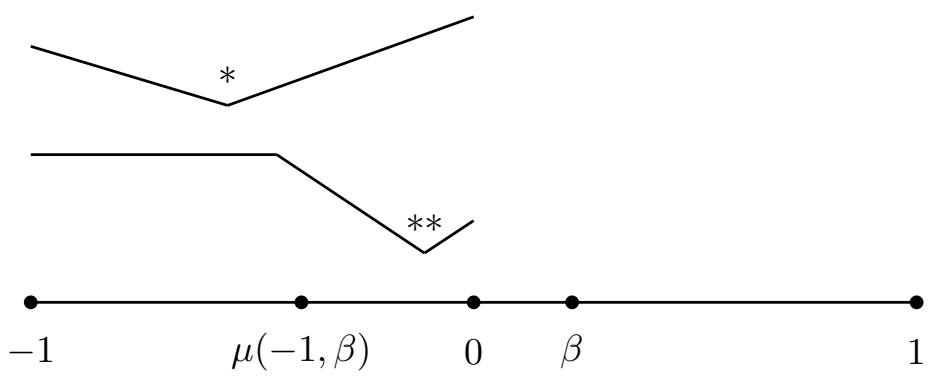

Figure 5.1: Examples of (*) Type-I and (**) Type-II preferences

Let $\beta$ be fixed, a solution if a function, say $\varphi, \varphi(p):[-1,0]^{N} \rightarrow[-1,0]$ that picks an outcome $\varphi(p)$ for any preference profile $p \in A_{L}^{N}$. Solution $\varphi$ is called

[AN] anonymous if for every profile $p \in A_{L}^{N}$ and permutation $\sigma$ over $N$, we have $\varphi\left(p_{\sigma}\right)=\varphi(p)$,

[PO] Pareto optimal if for every profile $p \in A_{L}^{N}$, agent $i \in N$ and alternative $x \in A$ with $x p^{*}(i) \varphi(p)$ there exists an agent $j \in N$ with $\varphi(p) p^{*}(j) x$,

[SP] strategy-proof if for every agent $i \in N$ and profiles $p, q \in A_{L}^{N}$ with $p(j)=q(j)$ for all $j \in N \backslash\{i\}$, we have $\varphi(p) p(i) \varphi(q)$.

We will define two more conditions on solutions to simplify our characterization. The first condition mentioned extends strategy-proofness such that a deviating agent cannot get an outcome which is further away although he might be indifferent. The second condition says that when agents are indifferent about two Pareto optimal alternatives, the one closer to 0 (the other country) should not be chosen. For example, when all agents are all indifferent between -1 and 0 , the condition implies that 0 should not be the outcome. A solution $\varphi$ satisfies

[SSP] strong strategy-proofness if for every agent $i \in N$, profiles $p, q \in$ $A_{L}^{N}$ with $p(j)=q(j)$ for all $j \in N \backslash\{i\}$, we have $|p(i)-\varphi(p)| \geq \mid p(i)-$ $\varphi(q) \mid$, 
[FA] far away condition if for every profile $p \in A_{L}^{N}$, Pareto optimal alternatives $x, y \in A_{L}$ with $x<y$ and all agents are indifferent between $x$ and $y$, we have $\varphi(p) \neq y$.

\subsection{The case $\beta=0$}

In this section, we investigate the case when Right locates its public bad on the boundary point zero. Note that, for any $p(i) \in[-0.5,0]$ we have $\{-1,0\} \subseteq \mathfrak{b}(p(i))$. Furthermore, for any $p(i) \in[-1,-0.5[$ we have $\mathfrak{b}(p(i))=$ $\{0\}$. Fig. 5.2 pictures examples of the preferences.

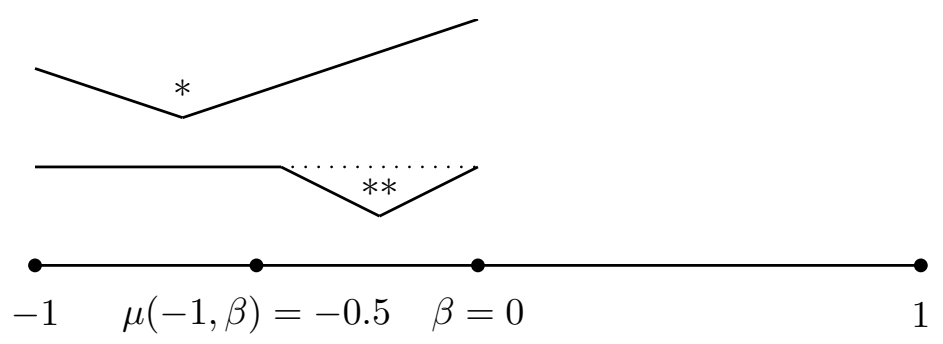

Figure 5.2: Examples of $(*)$ Type-I and $(* *)$ Type-II preferences when $\beta=0$

Now we will define a solution that picks 0 when it is the unique Pareto optimal point, and picks -1 for all other cases.

Definition 5.3.1. Let $\varphi^{*}$ be the solution defined as follows;

- $\varphi^{*}(p)=0$ if there exists an agent $i$ with $p(i) \in[-1,-0.5[$,

- $\varphi^{*}(p)=-1$ otherwise.

The reader may verify that $\varphi^{*}$ satisfies Pareto optimality, anonymity, strategy-proofness and the far away condition. Next we will show that $\varphi^{*}$ is the only solution that satisfies Pareto optimality and the far away condition.

Theorem 5.3.1. Let solution $\varphi$ satisfy $P O$ and $F A$. Then $\varphi=\varphi^{*}$. 
Proof. First, take any $p \in A_{L}^{N}$ with $p(i) \in[-1,-0.5[$ for some $i \in N$. Note that $\mathfrak{b}(p(i))=\{0\}$. Since $\mathfrak{b}(p(j))=\{0\}$ for all $p(j) \in[-1,-0.5[$ and $\{0\} \in \mathfrak{b}(p(k))$ for all $p(k) \in[-0.5,0]$, we have that point $\{0\}$ is the only Pareto optimal point for profile $p$. So $\varphi(p)=0=\varphi^{*}(p)$.

Second, take any $p \in A_{L}^{N}$ with $p(i) \in[-0.5,0]$ for all $i \in N$. Note that for any such preference $p(i)$ we have $\mathfrak{b}(p(i))=[-1, \kappa(p(i))] \cup\{0\}$. Pareto optimality implies $\varphi(p) \in[-1, \kappa(p)] \cup\{0\}$. Finally, the far away condition implies that $\varphi(p)=-1=\varphi^{*}(p)$, which completes the proof.

\subsection{The case $\beta \in] 0,1[,|N|=2$}

Throughout this section, we consider the case when Right locates its public bad in the interior of its region. Moreover, we will assume that there are two agents living in the left region. Let $N=\{j, k\}$.

First we will show that when there is an agent with preference of Type-II, then the outcome of a Pareto optimal and strategy-proof solution cannot be between the kink point of the profile and the boundary between the regions.

Lemma 5.4.1. Let solution $\varphi$ satisfy $P O$ and $S P$, and $p \in A_{L}^{N}$ be a profile with $N^{I I} \neq \emptyset$. Then $\left.\varphi(p) \notin\right] \kappa(p), 0[$.

Proof. To the contrary assume there exists $p \in A_{L}^{N}$ with $N^{I I} \neq \emptyset$ and $\varphi(p) \in] \kappa(p), 0\left[\right.$. W.l.o.g. let $k \in N^{I I}$ with $\kappa(p(k))=\kappa(p)$. Pareto optimality implies that $p(j) \in[-1, \mu(-1, \varphi(p))[$ and $|p(k)-\varphi(p)|>|p(k)-0|$, which gives $p(k) \in] \mu(\varphi(p), 0), 0]$. Now we will define three profiles;

- $p_{1}(j)=\mu(-1, \varphi(p))$ and $p_{1}(k)=p(k)$,

- $p_{2}(j)=p(j)$ and $p_{2}(k)=\mu(\varphi(p), 0)$,

- $p_{3}(j)=\mu(-1, \varphi(p))$ and $p_{3}(k)=\mu(\varphi(p), 0)$.

Step $1 \varphi\left(p_{1}\right)=-1$. Strategy-proofness implies that $\left|p(j)-\varphi\left(p_{1}\right)\right| \leq$ $|p(j)-\varphi(p)|$. Since $p(j) \in\left[-1, \mu(-1, \varphi(p))\left[\right.\right.$, we get $\varphi\left(p_{1}\right) \in[-1, \varphi(p)]$. Furthermore, strategy-proofness implies $\left|p_{1}(j)-\varphi\left(p_{1}\right)\right| \geq\left|p_{1}(j)-\varphi(p)\right|$. 
Since $p_{1}(j)=\mu(-1, \varphi(p))$, we get $\varphi\left(p_{1}\right)=-1$ or $\varphi\left(p_{1}\right)=\varphi(p)$. Since -1 Pareto dominates $\varphi(p)$ at profile $p_{1}$, this implies that $\varphi\left(p_{1}\right)=-1$.

$S$ tep $2 \varphi\left(p_{3}\right)=-1$. Note that, $-1 \in \mathfrak{b}(p(k))$. Strategy-proofness and $\varphi\left(p_{1}\right)=-1$ implies that $\left|p_{3}(k)-\varphi\left(p_{3}\right)\right| \geq\left|p_{3}(k)-\kappa\left(p_{3}(k)\right)\right|$. So $\varphi\left(p_{3}\right) \in$ $\left[-1, \kappa\left(p_{3}(k)\right)\right]$. Since $\left|p_{3}(k)-\varphi(p)\right|=\left|p_{3}(k)-0\right|$, we have $\kappa\left(p_{3}(k)\right) \in$ $\left[-1, \varphi(p)\left[\right.\right.$, which gives $\left|p_{3}(j)-x\right|<\left|p_{3}(j)-\varphi(p)\right|$ for all $x \in\left[-1, \kappa\left(p_{3}(k)\right)\right]$. Pareto optimality implies that $\varphi\left(p_{3}\right)=-1$.

$S$ tep $3 \varphi\left(p_{2}\right)=0$. Strategy-proofness implies that $\left|p(k)-\varphi\left(p_{2}\right)\right| \leq \mid p(k)-$ $\varphi(p) \mid$. Since $\left.p(k) \in] m_{\varphi(p), 0}, 0\right]$, we get $\varphi\left(p_{2}\right) \in[\varphi(p), 0]$. Furthermore, strategy-proof implies that $\left|p_{2}(k)-\varphi\left(p_{2}\right)\right| \geq\left|p_{2}(k)-\varphi(p)\right|$. Since $p_{2}(k)=$ $m_{\varphi(p), 0}$, we get $\varphi\left(p_{2}\right)=0$ or $\varphi\left(p_{2}\right)=\varphi(p)$. Since 0 Pareto dominates $\varphi(p)$ at profile $p_{2}$, this implies that $\varphi\left(p_{2}\right)=0$.

Since $\mathfrak{b}\left(p_{3}(j)\right)=\mathfrak{b}\left(p_{2}(j)\right)=0$, strategy-proof implies that $\varphi\left(p_{3}\right)=0$ which gives the contradiction.

Let $p \in A_{L}^{N}$ be such that $N^{I I}(p)=N$. Note that $[-1, \kappa(p)]$ is the set of all Pareto optimal points at this profile $p$. Notice that any arbitrary choice from this set can be used to define a new Pareto optimal and strategyproof solution, keeping the outcome stays the same for all other profiles. To simplify this, from now on we will require solutions to satisfy the far away condition.

Lemma 5.4.2. Let solution $\varphi$ satisfy $P O, S P$ and $F A$, and $p \in A_{L}^{N}$ be a profile with $N^{I I} \neq \emptyset$. Then $\left.\varphi(p) \notin\right]-1, \kappa(p)[$.

Proof. Let $p \in A_{L}^{N}$, and let $k \in N^{I I}(p)$ with $\kappa(p(k))=\kappa(p)$. So, $\mathfrak{b}(p(k))=$ $[-1, \kappa(p)]$. We distinguish the following four kinds of profiles based on $p(j)$.

- $p(j) \in[-1, \mu(-1, \kappa(p))]$. Note that $\kappa(p) p^{*}(j) x$ for all $\left.x \in\right]-1, \kappa(p)[$. Since $\mathfrak{b}(p(k))=[-1, \kappa(p)], \kappa(p)$ Pareto dominates any point in ]$1, \kappa(p)[$. So $\varphi(p) \notin]-1, \kappa(p)[$.

- $p(j) \in] \mu(-1, \kappa(p)), \mu(-1, \beta)]$. Note that $-1 p^{*}(j) x$ for all $\left.x \in\right]-$ $1, \kappa(p)[$. Since $\mathfrak{b}(p(k))=[-1, \kappa(p)],-1$ Pareto dominates any point in $]-1, \kappa(p)[$. So $\varphi(p) \notin]-1, \kappa(p)[$. 
- $p(j) \in] \mu(-1, \beta), p(k)[$. Note that this contradicts with $\kappa(p(k))=$ $\kappa(p)$.

- $p(j) \in[p(k), 0]$. Note that $[-1, \kappa(p)] \subseteq \mathfrak{b}(p(i))$. So both agents are indifferent between any points in $[-1, \kappa(p)]$. Far away condition implies $\varphi(p) \notin]-1, \kappa(p)[$.

So, we showed that $\varphi(p) \notin]-1, \kappa(p)[$.

Next we define the range of Pareto optimal, strategy-proof solutions that satisfy the far away condition.

Lemma 5.4.3. Let solution $\varphi$ satisfy $P O, S P$ and $F A$, and $p \in A_{L}^{N}$ be a profile. Then $\varphi(p) \in\{-1, \kappa(p), 0\}$ if $N^{I I}(p) \neq \emptyset$, and $\varphi(p) \in\{-1,0\}$ otherwise.

Proof. Let $p \in A_{L}^{N}$. If $N^{I I}(p) \neq \emptyset$, then Lemmas 5.4.1 and 5.4.2 imply that $\varphi(p) \in\{-1, \kappa(p), 0\}$.

Let $p \in A_{L}^{N}$ be such that $N^{I I}(p)=\emptyset$. In other words $p(i) \in[-1, \mu(-1, \beta)]$ for all $i \in N$. We will show that $\varphi(p) \in\{-1,0\}$. To the contrary, assume $\varphi(p) \in]-1,0[$. If there is no agent $i \in N$ with $p(i) \in[-1, \mu(-1, \varphi(p))[$, then 0 Pareto dominates $\varphi(p)$. So, that there exists an agent, $j$, with $p(j) \in[-1, \mu(-1, \varphi(p))[$. Similarly, if there is no agent $i \in N$ with $p(i) \in$ ]$\mu(\varphi(p), 0), 0]$, then 0 Pareto dominates $\varphi(p)$. So, that there exists an agent, $k$, with $p(k) \in] \mu(\varphi(p), 0), 0]$. Now we will define three profiles;

- $p_{1}(j)=\mu(-1, \varphi(p))$ and $p_{1}(k)=p(k)$,

- $p_{2}(j)=p(j)$ and $p_{2}(k)=\mu(\varphi(p), 0)$,

- $p_{3}(j)=\mu(-1, \varphi(p))$ and $p_{3}(k)=\mu(\varphi(p), 0)$.

Step $1 \varphi\left(p_{1}\right)=-1$. Strategy-proofness implies that $\left|p(j)-\varphi\left(p_{1}\right)\right| \leq \mid p(j)-$ $\varphi(p) \mid$. Furthermore, strategy-proof implies that $\left|p_{1}(j)-\varphi\left(p_{1}\right)\right| \geq \mid p_{1}(j)-$ $\varphi(p) \mid$. Since $p_{1}(j)=\mu(-1, \varphi(p))$, we get $\varphi\left(p_{1}\right)=-1$ or $\varphi\left(p_{1}\right)=\varphi(p)$. Since -1 Pareto dominates $\varphi(p)$ at profile $p_{1}$, this concludes that $\varphi\left(p_{1}\right)=$ -1 .

Step $2 \varphi\left(p_{3}\right)=-1$. Note that, $\mathfrak{b}\left(p_{3}(k)\right)=-1$. Strategy-proofness and $\varphi\left(p_{1}\right)=-1$ implies that $\varphi\left(p_{3}\right)=-1$. 
$S$ tep $3 \varphi\left(p_{2}\right)=0$. Strategy-proofness implies that $\left|p(k)-\varphi\left(p_{2}\right)\right| \leq \mid p(k)-$ $\varphi(p) \mid$. Furthermore, strategy-proof implies that $\left|p_{2}(k)-\varphi\left(p_{2}\right)\right| \geq \mid p_{2}(k)-$ $\varphi(p) \mid$. Since $p_{2}(k)=m_{\varphi(p), 0}$, we get $\varphi\left(p_{2}\right)=0$ or $\varphi\left(p_{2}\right)=\varphi(p)$. Since 0 Pareto dominates $\varphi(p)$ at profile $p_{2}$, this concludes that $\varphi\left(p_{2}\right)=0$.

$S$ tep $4 \varphi\left(p_{3}\right)=0$. Note that $\mathfrak{b}\left(p_{3}(j)\right)=\mathfrak{b}\left(p_{2}(j)\right)=0$, strategy-proof implies that $\varphi\left(p_{3}\right)=0$ which gives the contradiction with $\varphi\left(p_{3}\right)=-1$ from Step 2 .

So far we showed that solutions can only pick $\kappa(p)$ as "inner" outcome. Next we determine the profiles where this might happen. Moreover, we show that if it holds for such a profile, then it should hold for all such profiles.

Lemma 5.4.4. Let one solution $\varphi$ satisfy $P O, S P$ and $F A$, and $p \in A_{L}^{N}$ be a profile with $\varphi(p)=\kappa(p)$. Then one agent, say $k$, is Type-II with $\kappa(p(k))=$ $\kappa(p)$; and the other agent, say $j$, is Type-I with $p(j) \in[-1, \mu(-1, \kappa(p))[$.

Proof. Take any $p \in A_{L}^{N}$ with $\varphi(p)=\kappa(p)$. Let $k \in N^{I I}(p)$ with $\kappa(p(k))=$ $\kappa(p)$. The only thing remained to prove is $p(j) \in[-1, \mu(-1, \kappa(p))[$. To the contrary, assume not. Then $p(j) \in[\mu(-1, \kappa(p)), 0]$, which says that either agent $j$ is indifferent between -1 and $\kappa(p)$ if he/she has Type-II preference or agent $j$ strictly prefers -1 to $\kappa(p)$. Since $\{-1, \kappa(p)\} \subset \mathfrak{b}(p(k))$, we get that for the first case the far away condition implies that $\varphi(p) \neq \kappa(p)$, and for the second case -1 Pareto dominates $\kappa(p)$. So we reach a contradiction that ends this proof.

Let $\overline{A_{L}^{N}}=\left\{p \in A_{L}^{N} \mid\right.$ there exists one agent $k$ such that $\kappa(p(k))=\kappa(p)$, and there exists one agent $j$ such that $p(j) \in[-1, \mu(-1, \kappa(p))[\}$ denote the set of all profiles with a kink point. Note that $\varphi(p)=\kappa(p)$ implies $p \in \overline{A_{L}^{N}}$, but on forehand it is not clear whether the reverse holds. For instance take the solution $\varphi^{*}$ that picks 0 whenever it is Pareto optimal and -1 otherwise.

Next we will show that if a solution picks an inner outcome for some profile in $\overline{A_{L}^{N}}$, then it should choose an inner point for all profiles in $\overline{A_{L}^{N}}$. To reach this result, we will require a solution to satisfy one more condition; anonymity. 
Proposition 5.4.1. Let solution $\varphi$ satisfy $A N, P O, S P$ and $F A$, and $p \in$ $\overline{A_{L}^{N}}$ be a profile with $\varphi(p)=\kappa(p)$. Then $\varphi(q)=\kappa(q)$ for all $q \in \overline{A_{L}^{N}}$.

Proof. Let $p \in \overline{A_{L}^{N}}$ with $\kappa(p(k))=\kappa(p)$ and $p(j) \in[-1, \mu(-1, \kappa(p))]$. Take any $q \in \overline{A_{L}^{N}}$. We will show that $\varphi(q)=\kappa(q)$. By anonymity, let $\kappa(q(k))=\kappa(q)$ and $q(j) \in[-1, \mu(-1, \kappa(q))]$.

$S$ tep 1 Take $p^{\prime}(j)=-1$ and $p^{\prime}(k)=p(k)$. We will show that $\varphi\left(p^{\prime}\right)=\kappa\left(p^{\prime}\right)$. Note that, since $\left.\left.p^{\prime}(k)=p(k) \in\right] \mu(-1, \beta), 0\right]$ and $p^{\prime}(j) \in\left[-1, \mu\left(-1, \kappa\left(p^{\prime}\right)\right)\right]$ we have $p^{\prime} \in \overline{A_{L}^{N}}$. Moreover, $\kappa\left(p^{\prime}\right)=\kappa(p)$. Strategy-proofness and $\varphi(p)=\kappa(p)$ implies that $\left|p(j)-\varphi\left(p^{\prime}\right)\right| \leq|p(j)-\varphi(p)|$. Since $p(j) \in$ $[-1, \mu(-1, \kappa(p))]$, we get $\varphi\left(p^{\prime}\right) \in[-1, \varphi(p)]$. Furthermore, strategyproofness implies that $\left|p^{\prime}(j)-\varphi\left(p^{\prime}\right)\right| \geq\left|p^{\prime}(j)-\varphi(p)\right|$. So, $\varphi\left(p^{\prime}\right)=\kappa(p)=$ $\kappa\left(p^{\prime}\right)$.

$S$ tep 2 Take $r_{0}(j)=-1$ and $r_{0}(k)=0$. We will show that $\varphi\left(r_{0}\right)=\kappa\left(r_{0}\right)$. Note that $\kappa\left(p^{\prime}\right) \in \mathfrak{b}\left(r_{0}(k)\right)$. Strategy-proofness and $\varphi\left(p^{\prime}\right)=\kappa\left(p^{\prime}\right)$ trivially imply that $\varphi\left(r_{0}\right) \in\left[-1, \kappa\left(r_{0}\right)\right]$. Pareto-optimality and $r_{0}(j)=-1$ imply that $\varphi\left(r_{0}\right)=\kappa\left(r_{0}\right)$.

$S$ tep 3 Take $q^{\prime}(j)=-1$ and $\left.\left.q^{\prime}(k) \in\right] \mu(-1, \beta), 0\right]$. We will show that $\varphi\left(q^{\prime}\right)=$ $\kappa\left(q^{\prime}\right)$. For any profile, that will be defined in the following sub-steps, the dip of agent $j$ will remain at -1 .

By the previous step, we know that $\varphi\left(q^{\prime}\right)=\kappa\left(q^{\prime}\right)$ if $q^{\prime}(k)=0$. Let $\alpha \in$ $[\mu(-1, \beta), 0]$ be a minimal point such that for all $\left.\left.q^{\prime}(k) \in\right] \alpha, 0\right]$ we have $\varphi\left(q^{\prime}\right)=\kappa\left(q^{\prime}\right)$. We will show that $\alpha=\mu(-1, \beta)$. Assume to the contrary that $\alpha \neq \mu(-1, \beta)$. Consider $\left.\left.\alpha^{\prime} \in\right] \mu(-1, \beta), \alpha\right]$ which is very close to $\alpha$; $|0-\alpha|>\left|\alpha^{\prime}-\alpha\right|$. We will show that $\varphi\left(q_{x}\right)=\kappa\left(q_{x}\right)$ for all profiles $q_{x}$ with $q_{x}(j)=-1, q_{x}(k)=x$ with $\left.\left.x \in\right] \alpha^{\prime}, \alpha\right]$.

Consider profile $q_{\alpha}^{\varepsilon}$ where the dip of agent $k$ is close to $\left.\left.\alpha ; q_{\alpha}^{\varepsilon}(k) \in\right] \alpha, 0\right]$ with $\left|q_{\alpha}^{\varepsilon}(k)-\alpha\right|<\varepsilon$. Since $\left.\left.q_{\alpha}^{\varepsilon}(k) \in\right] \alpha, 0\right]$, we get $\varphi\left(q_{\alpha}^{\varepsilon}\right)=\kappa\left(q_{\alpha}^{\varepsilon}\right)$. Note that, $\left|q_{\alpha}(k)-\kappa\left(q_{\alpha}^{\varepsilon}\right)\right|>\left|q_{\alpha}(k)-0\right|$ and $\left|q_{\alpha}(k)-\kappa\left(q_{\alpha}\right)\right|>\left|q_{\alpha}(k)-\kappa\left(q_{\alpha}^{\varepsilon}\right)\right|$. Strategyproofness and $\varphi\left(q_{\alpha}^{\varepsilon}\right)=\kappa\left(q_{\alpha}^{\varepsilon}\right)$ imply that $\varphi\left(q_{\alpha}\right) \in\left\{-1, \kappa\left(q_{\alpha}\right)\right.$. Since -1 is Pareto dominated by $\kappa\left(q_{\alpha}\right)$ at profile $q_{\alpha}$, we get $\varphi\left(q_{\alpha}\right)=\kappa\left(q_{\alpha}\right)$.

Strategy proofness and $\varphi\left(q_{\alpha}\right)=\kappa\left(q_{\alpha}\right)$ imply that $\varphi\left(q_{x}\right) q_{x}(k) \varphi\left(q_{\alpha}\right)$. Since $\left.\left.q_{x}(k) \in\right] \mu\left(\kappa\left(q_{\alpha}\right), 0\right), 0\right]$, we have $\kappa\left(q_{\alpha}\right) q_{x}(k) 0$ and $\kappa\left(q_{x}\right) q_{x}(k) \kappa\left(q_{\alpha}\right)$. Further- 
more, Lemma 5.4.3 implies that $\varphi\left(q_{x}\right) \in\left\{-1, \kappa\left(q_{x}\right)\right\}$. But -1 is Pareto dominated by $\kappa\left(q_{X}\right)$ at profile $q_{x}$ since agent $k$ is indifferent between -1 and $\kappa\left(q_{x}\right)$, and $q_{x}(j)=-1$. So, $\varphi\left(q_{x}\right)=\kappa\left(q_{X}\right)$.

Step 4 We will show that $\varphi(q)=\kappa(q)$. Note that $\kappa\left(q^{\prime}\right)=\kappa(q)$ and $q^{\prime}(k)=$ $q(k)$. Strategy-proofness and $\varphi\left(q^{\prime}\right)=\kappa\left(q^{\prime}\right)$ imply that $\varphi(q) \in\{-1, \kappa(q)\}$. Since -1 is Pareto dominated by $\kappa(q)$, we have $\varphi(q)=\kappa(q)$.

Now we will show that there are only two solutions that satisfy AN, PO, $\mathrm{SP}$ and FA. The main difference between these solutions will be picking an inner point as outcome for some profile or not. Note that for any profile $p \in \overline{A_{L}^{N}}$, we have $\varphi(p) \in\{\kappa(p), 0\}$, because -1 is Pareto dominated by $\kappa(p)$. In view of Proposition 5.4.1, we define $\varphi_{1}$ as the solution that picks $\kappa(p)$ as the outcome, and $\varphi_{2}$ as the solution that picks 0 as the outcome for all profiles $p$ in $\overline{A_{L}^{N}}$.

Definition 5.4.1. Let $\varphi_{1}$ be the solution defined as follows;

- $\varphi_{1}(p)=\kappa(p)$ if $p \in \overline{A_{L}^{N}}$,

- $\varphi_{1}(p)=0$ if 0 is the unique Pareto optimal location,

- $\varphi_{1}(p)=-1$ otherwise.

Definition 5.4.2. Let $\varphi_{2}$ be the solution defined as follows;

- $\varphi_{2}(p)=-1$ if -1 is a Pareto optimal location while 0 is not or $p(i)=p(j)=-0.5$,

- $\varphi_{2}(p)=0$ otherwise.

Theorem 5.4.1. Let solution $\varphi$ satisfy AN, PO, SP and FA. Then $\varphi=\varphi_{1}$ or $\varphi=\varphi_{2}$.

Proof. First, let $k$ has preference of Type-II, $p(k) \in] \mu(-1, \beta), 0]$.

- $p(j) \in\left[-1, \mu(-1, \kappa(p))\left[\right.\right.$, then $p \in \overline{A_{L}^{N}}$. Since -1 is Pareto dominated by $\kappa(p)$ at any profile $p$ in $\overline{A_{L}^{N}}$, we have $\varphi(p) \in\{\kappa(p), 0\}$. In view of Proposition 5.4.1, either $\varphi(p)=\kappa(p)=\varphi_{1}(p)$ or $\varphi(p)=0=\varphi_{2}(p)$.

- $p(j)=\mu(-1, \kappa(p))$. By the previous part we know that $\varphi(q) \in$ $\{\kappa(q), 0\}$ when $q(j)=-1$ and $q(k) \in] \mu(-1, \beta), 0]$. Strategy-proofness 
and $\varphi(q)=\kappa(q)=\varphi_{1}(q)$ imply that $\varphi(p) \in\{-1, \kappa(p)\}$ and the far away condition implies that $v a(p)=-1=\varphi_{1}(p)$. Strategy-proofness and $\varphi(q)=0=\varphi_{2}(q)$ imply that $v a(p)=0=\varphi_{2}(p)$.

- $p(j) \in] \mu(-1, \kappa(p)),-0.5[$. Lemma 5.4 .3 implies $\varphi(p) \in\{-1,0\}$. Strategy-proofness and $\varphi(q)=\kappa(q)=\varphi_{1}(q)$ imply that $\varphi(p)=$ $-1=\varphi_{1}(p)$. Strategy-proofness and $\varphi(q)=0=\varphi_{2}(q)$ implies that $\varphi(p)=0=\varphi_{2}(p)$.

- $p(j) \in[-0.5, \mu(-1, \beta)]$. Then -1 is the only Pareto optimal point in $A_{L}$, so $\varphi(p)=-1=\varphi_{1}(p)=\varphi_{2}(p)$.

- $p(j) \in] \mu(-1, \beta), 0]$, then $[-1, \kappa(p)]$ are all the Pareto optimal points, the far away condition implies that $\varphi(p)=-1=\varphi_{1}(p)=\varphi_{2}(p)$.

Anonymity completes this part of characterization for profiles where at least one agent has Type-II preference. Now we will look at profiles where both agents have Type-I preferences.

- If both agents have dips in $[-1,-0.5[$, or one agent in $[-1,-0.5[$ and the other agent has -0.5 ; then 0 is the only Pareto optimal point. So, $\varphi(p)=0=\varphi_{1}(p)=\varphi_{2}(p)$.

- If both agents have -0.5 , then Pareto optimality implies $\varphi(p) \in$ $\{-1,0\}$. The far away condition implies that $\varphi(p)=-1=\varphi_{1}(p)=$ $\varphi_{2}(p)$. If both agents have dips in $\left.]-0.5, \mu(-1, \beta)\right]$, or one agent in ] $-0.5, \mu(-1, \beta)$ ] and the other agent has -0.5 ; then -1 is the only Pareto optimal point. So, $\varphi(p)=-1=\varphi_{1}(p)=\varphi_{2}(p)$.

- If one agent, say $j$, has dip in $[-1,-0.5[$ and the other agent, say $k$, has dip in $] 0.5, \mu(-1, \beta)]$. Lemma 5.4 .3 implies $\varphi(p) \in\{-1,0\}$. Let $p^{\prime}(j)=p(j)$ and $\left.\left.p^{\prime}(k) \in\right] \mu(-1, \beta), 0\right]$ with $\left|p^{\prime}(k)-\mu(-1, \beta)\right|<$ $|-0.5-p(k)|$. If $p^{\prime}(j) \in\left[-1, \mu(-1, \kappa(p))\left[\right.\right.$ then $\varphi\left(p^{\prime}\right)=\kappa\left(p^{\prime}\right)=\varphi_{1}\left(p^{\prime}\right)$ or $\varphi\left(p^{\prime}\right)=0=\varphi_{2}\left(p^{\prime}\right)$. Strategy-proofness and $\varphi\left(p^{\prime}\right)=\kappa\left(p^{\prime}\right)$ imply that $\varphi(p)=-1=\varphi_{1}(p)$. Strategy-proofness and $\varphi\left(p^{\prime}\right)=0$ imply that $\varphi(p)=0=\varphi_{2}(p)$. If $p^{\prime}(j) \in\left[\mu(-1, \kappa(p)),-0.5\left[\right.\right.$ then $\varphi\left(p^{\prime}\right)=-1=$ $\varphi_{1}\left(p^{\prime}\right)$ or $\varphi\left(p^{\prime}\right)=0=\varphi_{2}\left(p^{\prime}\right)$. Strategy-proofness and $\varphi\left(p^{\prime}\right)=-1$ imply that $\varphi(p)=-1=\varphi_{1}(p)$. Strategy-proofness and $\varphi\left(p^{\prime}\right)=0$ imply that $\varphi(p)=0=\varphi_{2}(p)$. 


\subsection{The case $\beta \in] 0,1[,|N|>2$}

In this section, we consider the case when Right locates its public bad at an inner point. Moreover, we assume that more than two agents live in the Left region.

Let $\varphi$ be a Pareto optimal, strong strategy-proof solution that satisfies the far away condition. We will show that Left can only locate its public bad at the boundary. Throughout the proof, we will use the following condition says a deviation done by a coalition cannot secure a location that is further away than the outcome, although the coalition is indifferent between this location and the outcome.

Intermediate Strong Strategy-proofness A solution $\varphi$ is called intermediate strong strategy-proof, if for all coalitions $S \subseteq N$ and all profiles $p$ and $q$ in $A_{L}^{N}$, such that $p(j)=q(j)$ for all $j \in N \backslash S$ and further such that there is an alternative $a$ with $p(i)=a$ for all $i \in S$, we have $|a-\varphi(p)| \geq|a-\varphi(q)|$.

Next we show the equivalence of strong strategy-proofness and intermediate strong strategy-proofness. We will use intermediate strong strategyproofness without mentioning it explicitly.

Lemma 5.5.1. Let $\beta \in\{0,1\}$. Then $\varphi$ is SSP if and only if ISSP.

Proof. The "If" part is straightforward. For the "Only if" part, let $\varphi$ be strong strategy-proof. Let $S$ be a coalition and $p, q \in A_{L}^{N}$ be profiles with $p(i)=a$ for any $a \in A_{L}$ and all $i \in S$ and $q(i)=p(i)$ for all $i \in N \backslash S$. To show $\varphi$ is intermediate strong strategy-proof, it is enough to show that $|a-\varphi(q)| \leq|a-\varphi(p)|$. W.o.l.g. let $S=\{1, \ldots, m\}$. Now we will define a series of profiles $q^{i}$ as follows; for any $i \in\{1, \ldots, m\}$ we have $q^{i}(i)=q(i)$, $q^{i}(j)=q^{i-1}(j)$ for all $j \in N \backslash\{i\}$, and $q^{0}=p$. Strong strategy-proofness implies that $\left|a-\varphi\left(q^{i}\right)\right| \leq\left|a-\varphi\left(q^{i-1}\right)\right|$ for all $i \in\{1, \ldots, m\}$. The transitivity of $\leq$ implies that $|a-\varphi(q)|=\left|a-\varphi\left(q^{m}\right)\right| \leq\left|a-\varphi\left(q^{0}\right)\right|=|a-\varphi(p)|$, hence completes the proof.

Now, we show that the range of Pareto optimal and strong strategy-proof solutions that satisfy the far-away condition is the boundaries of Left. 
Lemma 5.5.2. Let solution $\varphi$ satisfy $P O, S S P$ and $F A$, and $p \in A_{L}^{N}$ be a profile. Then $\varphi(p) \in\{-1,0\}$.

Proof. To the contrary assume there exists $p \in A_{L}^{N}$ and $\left.\varphi(p) \in\right]-1,0[$. Let $a=\mu(\varphi(p), 0)$, and $b=\mu(-1, \varphi(p))$. The far away condition and Pareto optimality implies that there exists $j \in N$ with $p(j) \in[-1, b[$ and $k \in N$ with $p(k) \in] a, 0]$. Let

- $\bar{a}, \bar{b} \in[-1,0]$ with $a \in] \bar{a}, 0]$ and $|a-\bar{a}|=\varepsilon$, and $b \in[-1, \bar{b}[$ and $|\bar{b}-b|=\varepsilon$ where $\varepsilon>0$ is a small number,

- $\left.\left.S_{1}=\{i \in N \mid p(i) \in] \bar{a}, 0\right]\right\}$ and $S_{2}=\{i \in N \mid p(i) \in[-1, \bar{b}[\}$,

- $p_{1} \in A_{L}^{N}$ with $p_{1}(i)=\bar{a}$ for all $i \in S_{1}$ and $p_{1}\left(i^{\prime}\right)=p\left(i^{\prime}\right)$ for all $i^{\prime} \in N \backslash S_{1}$,

- $p_{2} \in A_{L}^{N}$ with $p_{2}(i)=p(i)$ for all $i \in N \backslash S_{2}$ and $p_{2}\left(i^{\prime}\right)=\bar{b}$ for all $i^{\prime} \in S_{2}$,

- $p_{3} \in A_{L}^{N}$ with $p_{3}(i)=\bar{a}$ for all $i \in S_{1}, p_{3}\left(i^{\prime}\right)=\bar{b}$ for all $i^{\prime} \in S_{2}$ and $p_{3}(k)=p(k)$ for all $k \in N \backslash\left(S_{1} \cup S_{2}\right)$.

First we will show that $\varphi\left(p_{1}\right)=0$. Let $\left|S_{1}\right|=m$, and define a sequence $\tau$ over $S_{1}$. Now we will define a series of profiles $q^{i}$ as follows; for any $i \in\{1, \ldots, m\}$ we have $q^{i}(\tau(i))=0, q^{i}(j)=q^{i-1}(j)$ for all $j \in N \backslash\{\tau(i)\}$, and $q^{0}=p$. To show $\varphi\left(q^{m}\right)=\varphi(p)$ it is enough to show that if $\varphi\left(q^{i-1}\right)=\varphi(p)$ then $\varphi\left(q^{i}\right)=\varphi(p)$. Strong strategy-proofness and $\left.\left.q^{i-1}(i) \in\right] a, 0\right]$ imply that $\left|q^{i-1}(i)-\varphi\left(q^{i}\right)\right| \leq\left|q^{i-1}(i)-\varphi\left(q^{i-1}\right)\right|$, so $\varphi\left(q^{i}\right) \in\left[\varphi\left(q^{i-1}\right), 0\right]=[\varphi(p), 0]$. Furthermore, strong strategy-proof and $q^{i}(i)=0$ imply that $\mid q^{i}(i)-\varphi\left(q^{i}|\geq| q^{i}(i)-\varphi\left(q^{i-1}\right) \mid\right.$ which gives $\varphi\left(q^{i}\right)=\varphi\left(q^{i-1}\right)=\varphi(p)$. So, $\varphi\left(q^{m}\right)=\varphi(p)$.

Now we will show that $\varphi\left(p_{1}\right)=0$. Strong strategy-proofness and $q^{m}\left(S_{1}\right)=$ 0 imply that $\left|q^{m}\left(S_{1}\right)-\varphi\left(p_{1}\right)\right| \leq\left|q^{m}\left(S_{1}\right)-\varphi(p)\right|$, so we get $\varphi\left(p_{1}\right) \in[\varphi(p), 0]$. Since $p_{1}(i) \in[-1, a[$ for all $i \in N$ and $|\varphi(p)-a|=|a-0|$, we know that 0 Pareto dominates any point $x$ in $[\varphi(p), 0]$ at profile $p_{1}$. So, $\varphi\left(p_{1}\right)=0$. Similarly, one can show that $\varphi\left(p_{2}\right)=-1$. 
Next, we will show that $\varphi\left(p_{1}\right)=0$ implies that $\varphi\left(p_{3}\right)=0$. Let $\left|S_{1}\right|=m$, and define a sequence $\tau$ over $S_{1}$. Now we will define a series of profiles $r^{i}$ as follows; for any $i \in\{1, \ldots, m\}$ we have $r^{i}(\tau(i))=\bar{a}, r^{i}(j)=r^{i-1}(j)$ for all $j \in N \backslash\{\tau(i)\}$ and $r^{0}=p_{1}$. Note that $\varphi\left(r^{0}\right)=0$ and $r^{m}=p_{3}$. So to show $\varphi\left(r^{m}\right)=\varphi\left(p_{3}\right)=0$ it is enough to show that if $\varphi\left(r^{i-1}\right)=0$ then $\varphi\left(r^{i}\right)=0$. Strong strategy-proofness, $\varphi\left(r^{i-1}\right)=0$ and $0 \in \mathfrak{b}\left(r^{i}(i)\right)$ imply that $\varphi\left(r^{i}\right)=0$. So, $\varphi\left(p_{3}\right)=0$. Similarly, one can also show that $\varphi\left(p_{2}\right)=-1$ leads to $\varphi\left(p_{3}\right)=-1$, which gives contradiction that ends this proof.

Remark 5.5.1. Let solution $\varphi$ satisfy far away condition. Then $\varphi(-0.5)^{N}=-1$.

The reader may verify that Pareto optimal and strategy-proof solutions that satisfy the far away condition depend only on the individual preferences restricted to -1 and 0 . Now we will describe such solutions.

Let $\mathcal{W}_{-1}^{\varphi}$ be the set of pairs $(S, U) \in N \times N$ with $S \cap U=\emptyset$ and such that $\varphi(p)=-1$ for all profiles $p$ with $p(i) \in]-0.5,0]$ for all $i \in S$ and $p(i)=-0.5$ for all $i \in U$. Let $\mathcal{W}_{0}^{\varphi}$ be defined analogously. A pair $\left(\mathcal{W}_{-1}^{\varphi}, \mathcal{W}_{0}^{\varphi}\right)$ is

proper and strong, if either $(S, U) \in \mathcal{W}_{-1}^{\varphi}$ or $(T, U) \in \mathcal{W}_{0}^{\varphi}$ for all pairwise disjoint sets $S, U$ and $T$ with $S \cup T \cup U=N$,

Pareto optimal, if $(S, U) \in \mathcal{W}_{-1}^{\varphi}$ whenever $S \neq \emptyset$ and $S \cup U=N$, and $(T, U) \in \mathcal{W}_{0}^{\varphi}$ whenever $T \neq \emptyset$ and $T \cup U=N$,

monotone, if $\left(S^{\prime}, U^{\prime}\right) \in \mathcal{W}_{-1}^{\varphi}$ whenever $(S, U) \in \mathcal{W}_{-1}^{\varphi}$ and $S \subseteq S^{\prime}$ and $S \cup U \subseteq S^{\prime} \cup U^{\prime}$ and $\left(T^{\prime}, U^{\prime}\right) \in \mathcal{W}_{0}^{\varphi}$ whenever $(T, U) \in \mathcal{W}_{0}^{\varphi}$ and $T \subseteq T^{\prime}$ and $T \cup U \subseteq T^{\prime} \cup U^{\prime}$.

Theorem 5.5.1. The following holds;

(i) Let $\varphi$ be a Pareto optimal, strong strategy-proof solution, which satisfies the far away condition. Then $\left(\mathcal{W}_{-1}^{\varphi}, \mathcal{W}_{0}^{\varphi}\right)$ is proper and strong, Pareto optimal and monotone.

(ii) Let $\left(\mathcal{W}_{-1}, \mathcal{W}_{0}\right)$ be proper and strong, Pareto optimal, monotone and $(S, U) \in \mathcal{W}_{-1}$ when $U=N$. Then there is a Pareto optimal and 
strong strategy-proof solution $\varphi$, satisfying the far away condition, such that $\mathcal{W}_{-1}=\mathcal{W}_{-1}^{\varphi}$ and $\mathcal{W}_{0}=\mathcal{W}_{0}^{\varphi}$.

Since $\varphi$ depends only on the individual preferences between -1 and 0 we have that the pair $\left(\mathcal{W}_{-1}^{\varphi}, \mathcal{W}_{0}^{\varphi}\right)$ is both proper and strong. Pareto-optimality of $\varphi$ implies that the pair $\left(\mathcal{W}_{-1}^{\varphi}, \mathcal{W}_{0}^{\varphi}\right)$ is Pareto optimal. Furthermore, strategy-proofness of $\varphi$ implies that the pair $\left(\mathcal{W}_{-1}^{\varphi}, \mathcal{W}_{0}^{\varphi}\right)$ is monotone. It is straightforward to check that any proper and strong, Pareto optimal and monotone pair $\left(\mathcal{W}_{-1}, \mathcal{W}_{0}\right)$ with $(S, U) \in \mathcal{W}_{-1}$ when $U=N$ defines a Pareto optimal and strong strategy-proof rule $\varphi$, satisfying far away condition, with $\left(\mathcal{W}_{-1}^{\varphi}, \mathcal{W}_{0}^{\varphi}\right)=\left(\mathcal{W}_{-1}, \mathcal{W}_{0}\right)$

\subsection{The case $\beta=1$}

Let Right locate its public bad at 1 . We will show that Left can only locate the public bad at 0 or -1 . Note that all preferences are of Type-I since $\mu(-1, \beta)=\mu(-1,1)=0$. Before the proof we will define a condition that says a deviation by a coalition is not beneficial to that coalition.

Intermediate Strategy-proofness A solution $\varphi$ is called intermediate strategy-proof, if for all coalitions $S \subseteq N$ and all profiles $p$ and $q$ in $A_{L}^{N}$, such that $p(j)=q(j)$ for all $j \in N \backslash S$ and further such that there is an alternative $a$ with $p(i)=a$ for all $i \in S$, we have $\varphi(p) p(i) \varphi(q)$.

Since all preferences are of Type-I, i.e. preferences are strict on both sides of the dip; for any $a, b \in A_{L}, a p(i) b$ if and only if $|p(i)-b| \leq|p(i)-a|$. The reader can apply the proof of Lemma 5.5.1 to show the equivalence of strategy-proofness and intermediate strategy-proofness. We will use intermediate strong strategy-proofness without mentioning it explicitly.

Lemma 5.6.1. Let solution $\varphi$ satisfy $P O$ and $S P$, and $p \in A_{L}^{N}$ be a profile. Then $\varphi(p) \in\{-1,0\}$.

Proof. To the contrary assume there exists $p \in A_{L}^{N}$ and $\left.\varphi(p) \in\right]-1,0[$. Let $a=\mu(\varphi(p), 0)$, and $b=\mu(-1, \varphi(p))$. Pareto optimality implies that there exists at least one agent, say $j$, with $p(j) \in[-1, b[$ and at least 
one agent, say $k$, with $p(k) \in] a, 0]$. Let $\left.\left.S_{1}=\{i \in N \mid p(i) \in] a, 0\right]\right\}$ and $S_{2}=\{i \in N \mid p(i) \in[-1, b[\}$. Now we will define three profiles;

- $p_{1} \in A_{L}^{N}$ with $p_{1}(i)=a$ for all $i \in S_{1}$ and $p_{1}\left(i^{\prime}\right)=p\left(i^{\prime}\right)$ for all $i^{\prime} \in N \backslash S_{1}$,

- $p_{2} \in A_{L}^{N}$ with $p_{2}(i)=p(i)$ for all $i \in N \backslash S_{2}$ and $p_{2}\left(i^{\prime}\right)=b$ for all $i^{\prime} \in S_{2}$,

- $p_{3} \in A_{L}^{N}$ with $p_{3}(i)=a$ for all $i \in S_{1}, p_{3}\left(i^{\prime}\right)=b$ for all $i^{\prime} \in S_{2}$ and $p_{3}(k)=p(k)$ for all $k \in N \backslash\left(S_{1} \cup S_{2}\right)$.

First we will show that $\varphi\left(p_{1}\right)=0$. Let $\left|S_{1}\right|=m$, and w.l.o.g. suppose $S_{1}=\{1, \ldots, m\}$. Now we will define a series of profiles $q^{i}$ as follows; for any $i \in\{1, \ldots, m\}$ we have $q^{i}(\tau(i))=0, q^{i}(j)=q^{i-1}(j)$ for all $j \in N \backslash\{\tau(i)\}$, and $q^{0}=p$. To show $\varphi\left(q^{m}\right)=\varphi(p)$ it is enough to show that if $\varphi\left(q^{i-1}\right)=$ $\varphi(p)$ then $\varphi\left(q^{i}\right)=\varphi(p)$. Strategy-proofness and $\left.\left.q^{i-1}(i) \in\right] a, 0\right]$ imply that $\left|q^{i-1}(i)-\varphi\left(q^{i}\right)\right| \leq\left|q^{i-1}(i)-\varphi\left(q^{i-1}\right)\right|$, so $\varphi\left(q^{i}\right) \in\left[\varphi\left(q^{i-1}\right), 0\right]=[\varphi(p), 0]$. Furthermore, strategy-proof and $q^{i}(i)=0$ imply that $\mid q^{i}(i)-\varphi\left(q^{i}|\geq| q^{i}(i)-\right.$ $\varphi\left(q^{i-1}\right) \mid$ which gives $\varphi\left(q^{i}\right)=\varphi\left(q^{i-1}\right)=\varphi(p)$. So, $\varphi\left(q^{m}\right)=\varphi(p)$.

Now we will show that $\varphi\left(p_{1}\right)=0$. Strategy-proofness and $q^{m}\left(S_{1}\right)=0$ imply that $\left|q^{m}\left(S_{1}\right)-\varphi\left(p_{1}\right)\right| \leq\left|q^{m}\left(S_{1}\right)-\varphi(p)\right|$, so we get $\varphi\left(p_{1}\right) \in[\varphi(p), 0]$. Since $p_{1}(i) \in[-1, a]$ for all $i \in N$, there is at least one agent $j$ with $p(j) \in[-1, a[$ and $|\varphi(p)-a|=|a-0|$, we know that 0 Pareto dominates any point $x$ in $[\varphi(p), 0]$ at profile $p_{1}$. So, $\varphi\left(p_{1}\right)=0$. Similarly, one can show that $\varphi\left(p_{2}\right)=-1$.

Next, we will show that $\varphi\left(p_{1}\right)=0$ implies that $\varphi\left(p_{3}\right)=0$. Let $\left|S_{1}\right|=m$, and define a sequence $\tau$ over $S_{1}$. Now we will define a series of profiles $r^{i}$ as follows; for any $i \in\{1, \ldots, m\}$ we have $r^{i}(\tau(i))=a, r^{i}(j)=r^{i-1}(j)$ for all $j \in N \backslash\{\tau(i)\}$ and $r^{0}=p_{1}$. Note that $\varphi\left(r^{0}\right)=0$ and $r^{m}=p_{3}$. So to show $\varphi\left(r^{m}\right)=\varphi\left(p_{3}\right)=0$ it is enough to show that if $\varphi\left(r^{i-1}\right)=0$ then $\varphi\left(r^{i}\right)=0$. Strategy-proofness, $\varphi\left(r^{i-1}\right)=0$ and $0 \in \mathfrak{b}\left(r^{i}(i)\right)$ imply that $\varphi\left(r^{i}\right)=0$. So, $\varphi\left(p_{3}\right)=0$. Similarly, one can also show that $\varphi\left(p_{2}\right)=-1$ leads to $\varphi\left(p_{3}\right)=-1$, which gives contradiction that ends this proof.

Now we will describe Pareto optimal and strategy-proof solutions. Reasoning and notations are same as in the previous section, so we will only state 
the theorem.

Theorem 5.6.1. The following holds;

(i) Let $\varphi$ be a Pareto optimal, strategy-proof solution. Then $\left(\mathcal{W}_{-1}^{\varphi}, \mathcal{W}_{0}^{\varphi}\right)$ is proper and strong, Pareto optimal and monotone.

(ii) Let $\left(\mathcal{W}_{-1}, \mathcal{W}_{0}\right)$ be proper and strong, Pareto optimal, monotone. Then there is a Pareto optimal and strategy-proof solution $\varphi$, such that $\mathcal{W}_{-1}=\mathcal{W}_{-1}^{\varphi}$ and $\mathcal{W}_{0}=\mathcal{W}_{0}^{\varphi}$.

\subsubsection{Concluding Remarks}

The chapter considers the problem of locating a public bad along a region, Left, when there is a neighboring region, Right, with an existing public bad. Let $\beta$ be the location of the public bad of Right, then the results of this chapter can be summarized as follows;

- Let $\beta=0$. Then the solution $\varphi^{*}$, with $\varphi^{*}(p)=0$ if there exists an agent $i$ with $p(i) \in[-1,-0.5[$ and

- $\varphi^{*}(p)=-1$ otherwise, is the only Pareto optimal solution that satisfies the far away condition.

- Let $\beta \in] 0,1[$ with $|N|=2$. Then there are two anonymous, Pareto optimal and strategy-proof solutions, that satisfy the far away condition. The important thing that distinguishes these two solutions is that one of the solutions picks an inner point as the outcome for specific profiles.

- Let $\beta \in] 0,1[$ with $|N|>2$. Then the range of Pareto optimal and strong strategy-proof solutions that satisfy the far away condition is the boundaries, and we characterized all such solutions.

- Let $\beta=1$. Then the range of Pareto optimal and strategy-proof solutions that satisfy the far away condition is the boundaries, and we characterized all such solutions.

In this chapter we concentrated on the problem of locating a public bad for one of the two neighboring regions given a location of a public bad in the 
neighboring region. One possible extension of this study is that of locating a public bad for each of the two neighboring regions simultaneously. Another study could be that of locating a second public bad given the location of an other. 



\section{Bibliography}

Barbera S, Berga D, Moreno B (2012) Domains, ranges and strategyproofness: the case of single-dipped preferences. Social Choice and Welfare 39:335-352

Besfamille M, Lozachmeur JM (2010) NIMBY and mechanism design under different constitutional constraints. International Tax and Public Finance 17:114-132

Black D (1948) On the rationale of group decision-making. The Journal of Political Economy 56:23-34

Border KC, Jordan JS (1983) Straightforward elections, unanimity and phantom voters. Review of Economic Studies 50:153-170

Danilov VI (1994) The structure of non-manipulable social choice rules on a tree. Mathematical Social Sciences 27(2):123-131

Demange G (1982) Single-peaked orders on a tree. Mathematical Social Sciences 3(4):389-396.

Ehlers L (2002) Probabilistic allocation rules and single-dipped preferences. Social Choice and Welfare 19:325-348

Gibbard A (1973) Manipulation of voting schemes: a general result. Econometrica 41:587-602

Hansson B (1976) The existence of group preference functions. Public Choice 38, 89-98 
Inada K (1964) A note on the simple majority rule. Econometrica 31:525531

Kirman A, Sondermann D (1972) Arrow's theorem, many agents, and invisible dictators. Journal of Economic Theory 3:267-277

Klaus B (2001a) Target rules for public choice economies on tree networks and in Euclidean spaces. Theory and Decision 51(1):13-29

Klaus B (2001b) Coalitional strategy-proofness in economies with singledipped preferences and the assignment of an individual object. Games and Economic Behavior 34:64-82

Klaus B, Peters H, Storcken T (1997) Strategy-proof division of a private good when preferences are single-dipped. Economic Letters 55:339346

Kunreuther H, Kleindorfer PR (1986) A sealed-bid auction mechanism for siting noxious facilities. American Economic Review (Papers and Proceedings) 76:295-299

Lescop D (2007) Optimal mechanisms for siting noxious facilities. Review of Economic Design 10:273-284

Manjunath V (2009) Efficient and strategy-proof social choice when preferences are single-dipped. Working paper

Moulin H (1980) On strategy-proofness and single peakedness. Public Choice 35:437-455

Öztürk M, Peters H, Storcken T (2012) On the location of public bads: strategy-proofness under two-dimensional single-dipped preferences. Research Memorandum, Maastricht

Öztürk M, Peters H, Storcken T (2013) Strategy-proof location of a public bad on a disc. Economics Letters 119(1):14-16

Peremans W, Storcken T (1999) Strategy-proofness on single-dipped preference domains. In de Swart HMC (ed.) Logic, Game theory and Social choice. Tilburg University Press, The Netherlands 
Peters H, van der Stel H, Storcken, T (1992). Pareto Optimality, Anonymity, and Strategy-proofness in Location Problems. International Journal of Game Theory 21:221-235

Satterthwaite MA (1975) Strategy-proofness and Arrow's conditions: existence and correspondence theorem for voting procedures and social choice functions. Journal of Economic Theory 10:187-217

Schummer J, Vohra RV (2002) Strategy-proof location on a network. Journal of Economic Theory 104(2):405-428

Vohra RV (1999) The replacement principle and tree structured preferences. Economics Letters 63(2):175-180

Wistar R (1995) Orange County residents debate landfill site. The Chronicle 29 Sept. 1995. http://www.dukechronicle.com/articles/1995/09/29/orange-countyresidents-debate-landfill-site 11 June 2013 



\section{Nederlandse samenvatting (Dutch summary)}

Arrow's onmogelijkheidsstelling, welke min of meer betekent dat er geen goede collectieve voorkeursregel bestaat die met iedere denkbare combinatie van individuele voorkeuren rekening houdt, wordt gezien als de start van de moderne Sociale Keuze Theorie. Dit negatieve resultaat heeft tot veel vervolg onderzoek geleid dat vooral aantoonde hoe "sterk" dit resultaat was. Afzwakken van diverse gestelde condities of vervanging van deze door andere wenselijk te achten condities leidde tot eenzelfde conclusie. Een voorbeeld van dit laatste vormt de zogenaamde onmogelijkheidsstelling van Gibbard Satterthwaite. Gibbard (1973) en Satterthwaite (1975) hebben onafhankelijk van elkaar laten zien dat alleen dictatoriale collectieve keuzeregels, welke gedefinieerd zijn voor elke denkbare combinatie van individuele voorkeuren, Pareto optimaal en niet manipuleerbaar zijn. Hierbij betekent dat de collectieve keuzeregel Pareto optimaal is precies dan als er bij elke gegeven combinatie van individuele voorkeuren een uitkomst toegewezen wordt zodanig dat er geen ander mogelijk alternatief bestaat welk ieder individu prefereert bij die gegeven combinatie. De niet manipuleerbaarheidseis heeft alleszins te maken met het private karakter van de individuele voorkeuren. Een collectieve keuzeregel is manipuleerbaar als door eenzijdig afwijken van een individu bij een combinatie van individuele voorkeuren een voor dat individu betere uitkomst door die keuzeregel toegewezen wordt. Dit proefschrift behandelt collectieve keuzeregels waarbij de mogelijke voorkeuren van individuen beperkt zijn tot de verzameling 
van "single dipped" voorkeuren.

Een publiek "bad", bijvoorbeeld een windmolenpark, een coffeeshop, een vuilnisstortplaats of een nucleaire reactor, geeft aanleiding tot zo'n "single dipped" voorkeur. We stellen ons voor dat ieder individu een slechtst mogelijke locatie, de "dip", voor dit publiek "bad" voor ogen heeft, zoals bijvoorbeeld dat van zijn privé adres, de locatie van de school van zijn kinderen, of een locatie waarvan hij omwille van de natuur dit soort publieke "bad" niet zou willen. Verder veronderstellen we dat vanuit die "dip" de preferentie toeneemt met de afstand. Dit alles resulteert in een "single dipped" voorkeur. In dit proefschrift proberen we voor verschillende verzamelingen van alternatieven uit te zoeken welke Pareto optimale en niet manipuleerbare keuzeregels er bestaan als de voorkeuren van de individuen "single dipped" zijn. Voor de volledigheid vermelden we hier dat het in al deze gevallen een eindig aantal individuen betreft.

In Hoofdstuk 2 vormt de verzameling van alternatieven een polytoop in het platte vlak. Dus de "dips" van de individuen en de uitkomsten van de keuzeregel zijn punten van dit polytoop. Een "single best" punt van dit polytoop is een punt, zeg $x$ waarvoor er een "dip" bestaat, zeg $y$, zodat ten aanzien van de "single dipped" preferentie op basis van $y$ dat punt $x$ het enige beste punt is binnen het polytoop. Over het algemeen hebben niet manipuleerbare en Pareto optimale keuzeregels alleen "single best" punten als uitkomst. Bewezen wordt dat wanneer het polytoop precies twee "single best" punten heeft of wanneer het polytoop vier van zo'n punten heeft die in dat geval de vier hoekpunten van een rechthoek vormen, dan en alleen dan zijn er niet dictatoriale Pareto optimale en niet manipuleerbare keuzeregels mogelijk. Hierbij moet men denken aan monotone (eventueel) gewogen stemming tussen deze twee "single best" punten of tussen links en rechts en onder en boven bij vier "single best" punten. Dus indien er drie, vijf of meer "single best" punten zijn of wanneer er vier "single best" punten die niet de hoekpunten van een rechthoek vormen, dan is in dit geval van alternatieven een Pareto optimale en niet manipuleerbare keuzeregel dictatoriaal.

In Hoofdstuk 3 is de verzameling van alternatieven een cirkelschijf. Dit wil zeggen dat zowel de collectieve keuzes als de individuele "dips" punten van 
een cirkelschijf in het platte vlak zijn. Ook hier laten we zien dat alleen dictatoriale keuzeregels Pareto optimaal en niet manipuleerbaar zijn.

In Hoofdstuk 4 vormt een "tree" netwerk de verzameling van alternatieven. We laten eerst zien dat een Pareto optimale, niet corrupte anonieme en niet manipuleerbare keuzeregel enkel extreme "leaves" als uitkomst heeft. Dit zijn de uiteinden van een langst mogelijke pad over zo'n netwerk. Anoniem betekent dat de keuzeregel symmetrisch in haar argumenten is. Het maakt daarmee niet meer uit wie welke voorkeur heeft alleen hoe vaak een voorkeur genoemd is in een profiel. De stemming zou anoniem kunnen plaatsvinden. Niet corrupt betekent dat als een individu bij eenzijdig afwijken van alternatief $x$ naar alternatief $y$ kan switchen en dit individu is indifferent tussen deze beide alternatieven zowel ten aanzien van de originele als de afwijkende preferentie, dan is $x$ gelijk aan $y$. We laten vervolgens zien dat Pareto optimale, niet corrupte, anonieme en niet manipuleerbare keuzeregels precies de paarsgewijs monotone keuzeregels vormen. Paarsgewijze monotonie betekent dat de keuzeregel extreem punt $x$ toewijst zolang de onderliggende voorkeuren bij paarsgewijze vergelijking tussen de extreme punten voor $x$ niet verslechtert. Het toewijzen van de Condorcet winnaar, dit is het het extreem punt dat elk ander in paarsgewijze vergelijking verslaat, is een voorbeeld van een paarsgewijs monotone keuzeregel.

In hoofdstuk 5 bestuderen we een geval waarbij de alternatieven verzameling een gesloten lijnstuk is. Denk hierbij bijvoorbeeld aan het plaatsen van een windmolen park aan een kuststrook. Verder veronderstellen we dat op de kuststrook van een naburig land reeds een windmolenpark geplaatst is. $\mathrm{Nu}$ wordt de preferentie bepaald door de afstand tot het dichtstbijzijnde park. Hierdoor ontstaat er mogelijk een plateau aan een zijde van de "dip". Het geval van twee individuen wordt volledig onderzocht ten aanzien van de existentie van Pareto optimale en niet manipuleerbare keuzeregels. De uitkomst is echter technisch en we laten daarom hier een beschrijving achterwegen. 


\section{Short Curriculum Vitae}

Murat Öztürk was born on October 23, 1983 in Istanbul, Turkey. He received B.S. degree from Department of Mathematics at Bilkent University, Turkey in 2008. Then he attended M.Sc. program at Istanbul Bilgi University where he worked as research assistant in the project Computational Foundations of Social Choice supported by European Science Foundation and The Scientific and Technological Research Council of Turkey. He received his M.Sc. degree on August, 2010 with the thesis entitled On Refinements of Approval Voting. From September 2010 to November 2013, he worked as a Ph.D. candidate in Department of Quantitative Economics at Maastricht University, where he tutored Quantitative Methods, Management of Operations and Product Development lectures.

His main research topic is Social Choice Theory. He is also interested in Decision Theory and Game Theory. 